\title{
Homogeneous Gold Catalysis: Mechanistic Investigation of Hydroalkoxylation of Various Alkynes
}

\author{
by \\ Zhi Xiang Wong

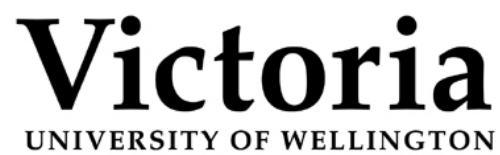 \\ Te Whare Wānanga \\ o te Ūpoko o te Ika a Māui \\ 59
}

\author{
A thesis \\ submitted to Victoria University of Wellington \\ in fulfilment of the \\ requirements for the degree of \\ Doctor of Philosophy \\ in Chemistry.
}

Victoria University of Wellington

2015 


\begin{abstract}
The reaction mechanism of the gold(III)-catalysed hydroalkoxylation of alkynes is studied to provide a deeper understanding of homogeneous gold catalysis. The study is conducted computationally using Density Functional Theory (DFT), with the PBE0 and BP86 functionals and basis sets of triple- $\zeta$ quality (aug-cc-pVTZ and aug-cc-pVTZ-PP for the gold atom). It emphasises the mechanisms undergone by various alkynes when they are activated by gold(III) catalysts towards nucleophilic attack to first form an enol ether and followed by a second nucleophilic attack to form a ketal as the final product. Hydrogen bonding networks formed by the solvent methanols are found to play a crucial role in the mechanism especially in the hydrogen migration steps that follow after the nucleophilic attacks. The first nucleophilic attacks are predicted to have rather low activation energies and hence they are expected to proceed fast while the second additions vary in activation barriers, depending on the steric effects in the substrates. The activation barrier for the last hydrogen migration is highest for all of the three reactions investigated and is expected to be the rate determining step. Investigations of internal alkyne reactions reveal that each elementary step requires a higher activation energy compared to terminal alkynes, which explains the low experimental rate of such reactions. Due to the regioselectivity problem in internal alkyne reactions, this results in a mixture of products which is difficult to isolate due to the similarities in their reaction energies. The study also highlights the calculated thermodynamics and kinetics of the reactions, which can be useful in predicting experimental outcomes. Arrhenius plots of concentration of each intermediate species against time were produced to further help the understanding of these mechanisms, whether or not the reactions go to full completion or stop at the formation of enol ether.
\end{abstract}




\section{Acknowledgments}

It was three years ago that my younger self decided to leave my comfort zone to study abroad in one of the windiest city in the world, Wellington. My $\mathrm{PhD}$ journey has been a great adventure filled with ups and downs that provided me not just the knowledge in my research field but also some important life lessons. These three years would never have worked out without the help of some individuals, to whom this page is dedicated to.

Undoubtedly, the first person I would like to express my thanks to is my supervisor, Matthias Lein for his patience, guidance, kindness and pretty much everything! He has been a great role model that I can look up to when I start my career as an academic. His supportive nature is not to be forgotten as he was always there when I needed his advice and in fact, he has never said 'no' to any opportunity that came my way. Matthias creates a relaxing and enjoyable environment in his group that balances both the social and work aspects, which makes a foreigner like me feels belonged.

Hassan, my previous supervisor from Universiti Sains Malaysia (USM), is someone who played an important role in my decision to pursue $\mathrm{PhD}$. He is also the very same person who recommended Matthias to me when I was searching for a potential supervisor. Even when we are in different continents, he never fails to give me the encouragement and confidence I needed to persist through my $\mathrm{PhD}$ research. I will always appreciate what he has done for me and as a promise to him, I will try my best to be the big 'tree' he hopes I'll become from the little 'seed' I once was.

My family, especially my parents have been very supportive of my decision to pursue PhD and I thank them for their blessings. All this time, they have always lent an ear to listen to all my complaints, share my joy when something works in my project and most importantly, all those Skype calls with them made me feel less homesick and more focussed in my work.

I would also like to thank my friends; those that are in Malaysia, those that I met in New Zealand and my groupmates for their presence in keeping my sanity intact. I was once told that $\mathrm{PhD}$ is a lonely journey where nobody else is doing the same project but I am sincerely glad to have my groupmates (Nina, Kevin, Ivan and Udbhav) around to lessen the loneliness of being a $\mathrm{PhD}$ candidate.

I would also like to acknowledge VUW for the Victoria Doctoral Scholarship that funded my study here in New Zealand. Additional computer time was provided by the VUW High Performance Computer Facilities Heisenberg and SciFacHPC. 


\section{Contents}

Abstract

Acknowledgments iii

Table of Contents iv

List of Figures vii

List of Schemes $\quad$ xi

List of Tables $\quad$ xii

1 Introduction 1

1.1 Reaction of alkynes with oxygen containing nucleophiles . . . . . . . . 4

1.2 Nucleophilic addition of unsaturated hydrocarbons $\ldots \ldots$. . . . . . . 7

1.3 Catalytic system . . . . . . . . . . . . . . . . . . 10

1.4 Comparison between gold and other transition metal catalysts . . . . . . . 17

1.5 Relativistic effects in late transition metal catalysts . . . . . . . . . . 22

1.6 Mechanistic investigation . . . . . . . . . . . . . . . 26

2 Methodology $\quad 40$

2.1 Computational chemistry methods . . . . . . . . . . . . . . . 40

2.2 Relativistic methods . . . . . . . . . . . . . . . . . . 46

2.3 Basis sets . . . . . . . . . . . . . . . . 48

2.4 Calculation algorithms and methods . . . . . . . . . . . . 51

2.5 Transition State Theory and reaction kinetics _ . . . . . . . . . . . 55 
2.5.1 Translational partition functions . . . . . . . . . . 57

2.5.2 Rotational partition functions . . . . . . . . . . 58

2.5.3 Vibrational partition function . . . . . . . . . . . 59

2.5.4 Electronic partition function . . . . . . . . . . . . 60

2.5 .5 Rate constant . . . . . . . . . . . . . . . 61

3 Results and Discussions (Reaction 1):

Mechanism of the Reaction of Propyne with Methanol Catalysed by $\mathrm{AuCl}_{3} \quad 63$

3.1 Reaction sequence $\ldots \ldots \ldots \ldots \ldots$

3.1 .1 Pathway $3 \mathrm{MeOH}-\mathrm{s} \ldots \ldots \ldots 65$

3.1 .2 Pathway $2 \mathrm{MeOH}-\mathrm{s} \ldots \ldots \ldots 66$

3.1 .3 Hydrogen migration $\ldots \ldots \ldots 6$

3.1.4 Second nucleophilic attack . . . . . . . . . . . . . 69

3.1.5 Comparison between singlet and triplet states . . . . . . . . 72

3.2 Comparison of $\mathrm{H}_{2} \mathrm{O}$ - and $\mathrm{CH}_{3} \mathrm{OH}$-nucleophilic addition mechanisms . . . 72

3.3 Conclusions . . . . . . . . . . . . . . . . . . . . 74

4 Results and Discussions (Reaction 2):

Mechanism of the Reaction of 4,4-dimethylpent-2-yne with Methanol Catalysed by $\mathrm{AuCl}_{3}$

4.1 Reaction sequence . . . . . . . . . . . . . . . . 75

4.1.1 Mechanism 1, M1 . . . . . . . . . . . . 77

4.1.2 Mechanism 2, M2 ................. 80

4.1.3 Comparison between singlet and triplet states . . . . . . . . 86

4.2 Comparison of the two mechanisms, M1 and M2 f . . . . . . 87

4.3 Conclusions .............................. 91 
5 Results and Discussions (Reaction 3):

Mechanism of the Reaction of Phenylacetylene with Methanol Catalysed by $\mathrm{Au}\left(\mathrm{C}_{6} \mathbf{F}_{5}\right) \mathrm{Cl}_{2}$

5.1 Reaction sequence $\ldots \ldots \ldots \ldots$. . . . . . . . . . . 93

5.1.1 Pathway $3 \mathrm{MeOH}-\mathrm{L} \ldots \ldots \ldots . \ldots . \ldots 94$

5.1 .2 Pathway $2 \mathrm{MeOH}-\mathrm{L} \ldots \ldots \ldots . \ldots . \ldots 95$

5.1.3 Hydrogen migration and second nucleophilic attack . . . . . . . 96

5.2 Conclusions . . . . . . . . . . . . . . . . . . 101

6 Discussions: Comparison of the Three Reactions 102

6.1 A comparison between terminal and internal alkynes . . . . . . . . 102

6.2 Thermodynamics of the reactions . . . . . . . . . . . . 105

6.3 Reaction kinetics . . . . . . . . . . . . . . . . . . 108

$\begin{array}{lll}7 & \text { Summary and Outlook } & 117\end{array}$

$\begin{array}{lr}\text { Appendix A } & \mathbf{1 2 0}\end{array}$

$\begin{array}{ll}\text { Bibliography } & 123\end{array}$ 


\section{List of Figures}

1.1 The correlation of hydrochlorination of activity of metal chlorides with the standard electrode potential. (Reproduced with permission; from Hutchings ${ }^{22}$ copyright (c)1985 Elsevier.) . . . . . . . . . . . . . . . . . . . .

1.2 Selectivity problems in alkenes, alkynes and allenes. The black and red arrows represent the two possibilities of nucleophile attack at different positions while the blue arrows represent single nucleophile addition in alkynes and allenes. .

1.3 The deactivated gold(I) trimer observed by Khin et al. (Reproduced with permission; from Khin et al. ${ }^{53}$ copyright (c) 2010 Wiley.) . . . . . . . . . . . .

1.4 Structures of $\mathrm{Pt}, \mathrm{Au}$ and $\mathrm{Hg}$ complexes. (Reproduced with permission; from Leyva-Pérez and Corma ${ }^{50}$ copyright (c) 2012 Wiley.) . . . . . . . . . . . . . . 20

1.5 The LUMO density of Au(left) and Pt(right) complex. (Reproduced with permission; from Pernpointner and Hashmi ${ }^{102}$ copyright (C)2009 American Chemical Society.) . . . . . . . . . . . . . . . . .

1.6 The ratio of relativistic $\left(<\mathrm{r}>_{\text {rel. }}\right)$ and non-relativistic $\left.(<\mathrm{r}\rangle_{\text {non-rel. }}\right) 6 s$-shell radii in atomic ground state. (Reproduced with permission; from Pyykkö and Desclaux ${ }^{104}$ copyright (c)1979 American Chemical Society.) . . . . . . . . . . 23

1.7 Relativistic and non-relativistic energy level diagrams of $5 d, 6 s$ and $6 p$ orbitals. . 24

1.8 The connections between past research and their contribution to this study. . . . 28

1.9 The mechanism for the first addition of methanol to 1,2-diphenylacetylene using $\left[\left(\mathrm{Ph}_{3} \mathrm{P}\right)-\mathrm{Au}\right]^{+}$complex as catalyst. Energies are in $\mathrm{kcal} \mathrm{mol}^{-1}$. (Reproduced with permission; from Mazzone et al. ${ }^{116}$ copyright (C)2012 American Chemical Society.) . . . . . . . . . . . . . . . . . . .

1.10 The mechanism for the second nucleophilic addition of water to both enol ether $\mathrm{E}$ (solid line) and enol ether $\mathrm{Z}$ (dashed line) isomers using $\left[\left(\mathrm{Ph}_{3} \mathrm{P}\right)-\mathrm{Au}\right]^{+}$complex as catalyst. Energies are in $\mathrm{kcal} \mathrm{mol}^{-1}$. (Reproduced with permission; from Mazzone et al. ${ }^{116}$ copyright (c) 2012 American Chemical Society.) . . . . . . . 32

1.11 The reaction mechanism of water addition to propyne catalysed by $\mathrm{AuCl}_{3}$. Energies are in $\mathrm{kcal} \mathrm{mol}^{-1}$ and bond lengths are in $\AA$. (Reproduced with permission; from Lein et al. ${ }^{117}$ copyright (C)2010 American Chemical Society.) .

1.12 Energy profile of $\mathrm{Au}(\mathrm{I})$ catalysed addition of water to propyne (without assistance by water). Energies are in $\mathrm{kcal} \mathrm{mol}^{-1}$ and bond lengths are in $\AA$. (Reproduced with permission; from Krauter et al. ${ }^{115}$ copyright (c)2010 Wiley.) . 
1.13 Energy profile of $\mathrm{Au}(\mathrm{I})$ catalysed addition of water to propyne (assisted by water). Energies are in $\mathrm{kcal} \mathrm{mol}^{-1}$ and bond lengths are in $\AA$. (Reproduced with permission; from Krauter et al. ${ }^{115}$ copyright (C) 2010 Wiley.) . . . . . . . . 38

2.1 Jacob's ladder of chemical accuracy. . . . . . . . . . . . . . . . . . . . . 44

2.2 Mean signed and unsigned deviations of calculated bond dissociation energies with various DFT/ATZ and ab initio methods taking $\mathrm{CCSD}(\mathrm{T}) / \mathrm{CBS}_{\mathrm{final}}$ as

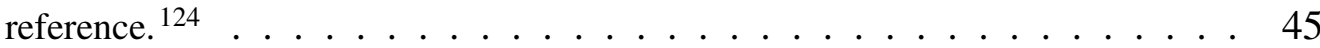

2.3 The difference between Schrödinger and Dirac solutions. . . . . . . . . . . . . 47

2.4 Location of a saddle point in the energy profile. . . . . . . . . . . . 53

2.5 A typical energy profile along the reaction coordinate. . . . . . . . . . . . 54

3.1 The perpendicular, $\boldsymbol{2}^{\prime}{ }_{\mathrm{s}}$ and in-plane, $\boldsymbol{2}_{\mathrm{s}}$ conformations of $\mathrm{AuCl}_{3}$-propyne activated complex. All energetic changes from PBE0 and BP86 calculations are given in $\mathrm{kcal} \mathrm{mol}^{-1}$. Values for BP86 calculations are given in parentheses. . . .

3.2 First part of the mechanism. Relative energies with respect to the starting materials of PBE0 calculations are given below structures $\mathbf{1}_{\mathrm{S}}$ to $\mathbf{6}_{\mathrm{S}}$ respectively while energetic changes with respect to the previous structures are given on the dotted lines that represent the reaction coordinate. Values for BP86 calculations are given in parentheses. The structural parameters are in $\AA$. All energies in $\mathrm{kcal} \mathrm{mol}^{-1}$

3.3 Second part of the mechanism. Relative energies with respect to the starting materials of PBE0 calculations are given below structures $\mathbf{6}_{\mathrm{s}}$ to $\mathbf{7}_{\mathrm{s}}$ respectively while energetic changes with respect to the previous structures are given on the dotted lines that represent the reaction coordinate. Values for BP86 calculations are given in parentheses. The structural parameters are in $\AA$. All energies in $\mathrm{kcal}^{\mathrm{mol}}{ }^{-1} \ldots \ldots \ldots \ldots \ldots \ldots \ldots \ldots$

3.4 Third part of the mechanism. Relative energies with respect to the starting materials of PBE0 calculations are given below structures $\mathbf{7}_{\mathrm{S}}$ to the product respectively while energetic changes with respect to the previous structures are given on the dotted lines that represent the reaction coordinate. Values for BP86 calculations are given in parentheses. The structural parameters are in $\AA$. All energies in $\mathrm{kcal} \mathrm{mol}^{-1} \ldots \ldots \ldots \ldots$. . . . . . . . . . . 70

3.5 The overall most probable mechanism for $\mathrm{AuCl}_{3}$-catalysed reaction of propyne and methanol with the explicit participation of solvent molecules. Values for BP86 calculations are given in parentheses. The structural parameters are in $\AA$. All energies in $\mathrm{kcal} \mathrm{mol}^{-1} \ldots \ldots \ldots \ldots 71$ 
4.1 The numbering of carbon atoms in the reactant. . . . . . . . . . . . 75

4.2 The perpendicular, $\mathbf{2}_{\mathrm{m}}$ and in-plane conformations, $\mathbf{2}_{\mathrm{m}}^{\prime}$ and $\mathbf{2}^{\prime \prime}{ }_{\mathrm{m}}$ of $\mathrm{AuCl}_{3}-$ heptyne activated complex. The energetic changes from PBE0 calculations are given in $\mathrm{kcal} \mathrm{mol}^{-1}$. . . . . . . . . . . . . . . . . . 76

4.3 First part of the Mechanism 1 (M1). Relative energies with respect to the starting materials of PBE0 calculations are given below structures $\mathbf{1}_{\mathrm{m}}$ to $\mathbf{7 - \mathbf { 1 } _ { \mathrm { m } }}$ respectively while energetic changes with respect to the previous structures are given on the dotted lines that represent the reaction coordinate. The structural parameters are in $\AA$. All energies in $\mathrm{kcal} \mathrm{mol}^{-1}$. Some methyl groups are depicted in tube format for clarity. . . . . . . . . . . . . . . . 78

4.4 Second part of the Mechanism 1 (M1). Relative energies with respect to the starting materials of PBE0 calculations are given below structures $\mathbf{7 - 1} \mathbf{m}_{\mathrm{m}}$ to the product respectively while energetic changes with respect to the previous structures are given on the dotted lines that represent the reaction coordinate. The structural parameters are in $\AA$. All energies in $\mathrm{kcal} \mathrm{mol}^{-1}$. Some methyl groups are depicted in tube format for clarity. . . . . . . . . . . . . 81

4.5 First part of the Mechanism 2 (M2). Relative energies with respect to the starting materials of PBE0 calculations are given below structures $\mathbf{1}_{\mathrm{m}}$ to $\mathbf{7 - \mathbf { 2 } _ { \mathrm { m } }}$ respectively while energetic changes with respect to the previous structures are given on the dotted lines that represent the reaction coordinate. The structural parameters are in $\AA$. All energies in $\mathrm{kcal} \mathrm{mol}^{-1}$. Some methyl groups are depicted in tube format for clarity. . . . . . . . . . . . . . . .

4.6 Second part of the Mechanism 2 (M2). Relative energies with respect to the starting materials of PBE0 calculations are given below structures $\mathbf{7 - 2} \mathrm{m}$ to the product respectively while energetic changes with respect to the previous structures are given on the dotted lines that represent the reaction coordinate. The structural parameters are in $\AA$. All energies in $\mathrm{kcal} \mathrm{mol}^{-1}$. Some methyl groups are depicted in tube format for clarity. . . . . . . . . . . . . .

4.7 The overall processes of Mechanism 1 (M1) for $\mathrm{AuCl}_{3}$-catalysed reaction of 4,4-dimethylpent-2-yne and methanol with the explicit participation of solvent molecules. The structural parameters are in $\AA$. All energies in $\mathrm{kcal} \mathrm{mol}^{-1}$. Some methyl groups are depicted in tube format for clarity. . . . . . . . . . . . . .

4.8 The overall processes of Mechanism 2 (M2) for $\mathrm{AuCl}_{3}$-catalysed reaction of 4,4-dimethylpent-2-yne and methanol with the explicit participation of solvent molecules. The structural parameters are in $\AA$. All energies in $\mathrm{kcal} \mathrm{mol}^{-1}$. Some methyl groups are depicted in tube format for clarity. . . . . . . . . . . 
5.1 The perpendicular, $\mathbf{2}_{\mathrm{L}}$ and in-plane conformations, $\mathbf{2}_{\mathrm{L}}^{\prime}$ of $\mathrm{Au}\left(\mathrm{C}_{6} \mathrm{~F}_{5}\right) \mathrm{Cl}_{2}$-phenylacetylene activated complex. The energetic change from PBE0 calculation is given

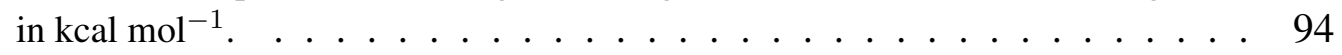

5.2 First part of the mechanism. Relative energies with respect to the starting materials from PBE0 calculations are given below structures $\mathbf{1}_{\mathrm{L}}$ to $\mathbf{6}_{\mathrm{L}}$ respectively while energetic changes with respect to the previous structures are given on the dotted lines that represent the reaction coordinate. The structural parameters are in $\AA$. All energies in $\mathrm{kcal} \mathrm{mol}^{-1}$. . . . . . . . . . . . . . . . . . . . .

5.3 Third part of the mechanism. Relative energies with respect to the starting materials of PBE0 calculations are given below structures $\mathbf{6}_{\mathrm{L}}$ to the product respectively while energetic changes with respect to the previous structures are given on the dotted lines that represent the reaction coordinate. The structural parameters are in $\AA$. All energies in $\mathrm{kcal} \mathrm{mol}^{-1}$. . . . . . . . . . . . . . 98

5.4 The overall mechanism for $\mathrm{Au}\left(\mathrm{C}_{6} \mathrm{~F}_{5}\right) \mathrm{Cl}_{2}$-catalysed reaction of phenylacetylene and methanol with the explicit participation of solvent molecules. The structural parameters are in $\AA$. All energies in $\mathrm{kcal} \mathrm{mol}^{-1}$. . . . . . . . . . . . . . . . 100

6.1 The plots of concentration of intermediates $\left(\mathrm{mol} \mathrm{dm}^{-3}\right)$ against time (s) of Reaction 1. The smaller graph on the left represent how the plots look like on a smaller scale. . . . . . . . . . . . . . . . . . .

6.2 The plots of concentration of intermediates $\left(\mathrm{mol} \mathrm{dm}^{-3}\right)$ against time $(\mathrm{s})$ of Reaction 2-M1. The smaller graph on the left represent how the plots look like on a smaller scale. . . . . . . . . . . . . . . . . . . . . 114

6.3 The plots of concentration of intermediates $\left(\mathrm{mol} \mathrm{dm}^{-3}\right)$ against time (s) of Reaction 2-M2. The smaller graph on the left represent how the plots look like on a smaller scale. . . . . . . . . . . . . . . . . . .

6.4 The plots of concentration of intermediates $\left(\mathrm{mol} \mathrm{dm}^{-3}\right)$ against time (s) of Reaction 3. The smaller graph on the left represent how the plots look like on a smaller scale . . . . . . . . . . . . . . . . . . . . 116

A.1 Rate constant values represented on a logarithmic scale with $k_{\min }$ being the lowest value while $k_{\max }$ being the largest value in the set. . . . . . . . 120

A.2 The assignment of $k_{\min }$ and $k_{\max } \ldots \ldots \ldots \ldots \ldots \ldots \ldots$

A.3 The range of the rate constants, $k$ and relative rate constants, $k^{\prime}$ on logarithmic scale . . . . . . . . . . . . . . . . . . 121

A.4 The $k$ values are projected on the smaller scale and $k^{\prime}$ values are calculated. . . . 122 


\section{List of Schemes}

1.1 Reaction scheme for the formation of ketones and ketals from unactivated alkynes. 4

1.2 The possible intermediates proposed in the reaction mechanism involving allenes. $\eta^{2}$-complex (I) and carbocation (II) intermediates. . . . . . . . . . . 9

1.3 The possible intermediates proposed in the reaction mechanism involving alkynes. Carbene (III) and carbocation (IV) intermediates. . . . . . . . . . 15

1.4 Possible interaction between gold(I) catalyst and terminal alkyne. (Reproduced with permission; from Velegraki and Stratakis ${ }^{89}$ copyright (c)2013 American Chemical Society.) . . . . . . . . . . . . . . . . 16

1.5 Rearrangment of vinyl carbocation to form an allene structure. (Reproduced with permission; from Velegraki and Stratakis ${ }^{89}$ copyright (C)2013 American Chemical Society.) . . . . . . . . . . . . . . . . 16

1.6 Suggested reaction pathway in the formation of anti-Markovnikov hydration product. (Reproduced with permission; from Tokunaga and Wakatsuki ${ }^{99}$

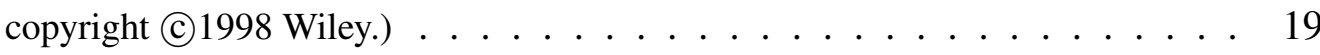

1.7 Reaction mechanism for the addition of methanol to propyne proposed by Teles and coworkers. (Reproduced with permission; from Teles et al. ${ }^{28}$ copyright (c)1998 Wiley.) . . . . . . . . . . . . . . . . . . . . . . 27

1.8 Plausible mechanism for the hydration of alkynes using $\mathrm{AuPR}_{3}{ }^{+}$catalyst. (Reproduced with permission; from Leyva-Pérez and Corma ${ }^{56}$ copyright (C)2009 American Chemical Society.) . . . . . . . . . . . . . . . . 30

1.9 Schematic reaction mechanism proposed by Laguna and coworkers. (Reproduced with permission; from Casado et al. ${ }^{25}$ copyright (c)2003 American Chemical Society.) . . . . . . . . . . . . . . . 33 


\section{List of Tables}

3.1 Energy difference, $\Delta \mathrm{E}$ between triplet and singlet state structures computed at PBE0 and BP86 functional. All values are in $\mathrm{kcal} \mathrm{mol}^{-1} \ldots \ldots$. . . . . . . . 72

4.1 Energy difference, $\Delta \mathrm{E}$ between triplet and singlet state structures computed at PBE0 functional. All values are in $\mathrm{kcal} \mathrm{mol}^{-1} \ldots \ldots$. . . . . . . . . . . 87

5.1 The energetical difference ( $\mathrm{kcal} \mathrm{mol}^{-1}$ ) between the two methods (PBE0/aug-cc-pVTZ and PBE0/aug-cc-pVTZ//PBE0/aug-cc-pVDZ) used in the calculations. . . . . . 93

6.1 Overall Gibbs free energy changes, $\Delta \mathrm{G}_{\mathrm{r}}$ of all reactions under study computed with PBE0 functional. All values are in $\mathrm{kcal} \mathrm{mol}^{-1}$. . . . . . . . . . . . 105

6.2 The energetic differences, $\Delta \mathrm{E}$ and Gibbs free energy changes, $\Delta \mathrm{G}_{\text {release }}$ for the release product by elimination of catalyst and extra solvent molecule (last step of reaction) computed with PBE0 functional. All values are in $\mathrm{kcal} \mathrm{mol}^{-1}$. . . 106

6.3 Gibbs free energy changes, $\Delta \mathrm{G}_{\text {enol }}$ for the formation of enol ether computed with PBE0 functional. All values are in $\mathrm{kcal} \mathrm{mol}^{-1}$. . . . . . . . . . . 107

6.4 The energetic differences, $\Delta \mathrm{E}$ and Gibbs free energy changes, $\Delta \mathrm{G}_{\text {enol-rel }}$ for the release of the enol ether intermediate by elimination of catalyst and extra solvent molecule computed with PBE0 functional. All values are in $\mathrm{kcal} \mathrm{mol}^{-1}$. . . . . 107

6.5 Rate constant values, $k\left(\mathrm{~s}^{-1}\right)$ and the corresponding relative values, $k^{\prime}\left(\mathrm{s}^{-1}\right)$ for Reaction 1. . . . . . . . . . . . . . . . . . 110

6.6 Rate constant values, $k\left(\mathrm{~s}^{-1}\right)$ and the corresponding relative values, $k^{\prime}\left(\mathrm{s}^{-1}\right)$ for Reaction 2-M1. . . . . . . . . . . . . . . . . . . . . . 110

6.7 Rate constant values, $k\left(\mathrm{~s}^{-1}\right)$ and the corresponding relative values, $k^{\prime}\left(\mathrm{s}^{-1}\right)$ for Reaction 2-M2 . . . . . . . . . . . . . . . . . . . . . 111

6.8 Rate constant values, $k\left(\mathrm{~s}^{-1}\right)$ and the corresponding relative values, $k^{\prime}\left(\mathrm{s}^{-1}\right)$ for Reaction 3. . . . . . . . . . . . . . . . . 111 


\section{Chapter 1}

\section{Introduction}

Transition metal catalysis has been one of the leading fields in chemistry that contributes to the advancements in chemical reactions we see today. The field has opened up many gates in chemical reactions with their ability to catalyse numerous organic and inorganic reactions, especially those that were not feasible without the presence of the catalyst itself. Transition metals are good candidates for the development of catalysts because of their ability to adopt multiple oxidation states that alter the electronic properties of the reaction. This effect could potentially increase the rate of reaction and subsequently increase the efficiency in forming the products which is highly desirable in industry. They are also able to coordinate with other substrates through dative bonds to activate the substrate in a reaction. Kinetically, a chemical reaction occurs when the reactants come into contact in a specific orientation and the rate of the reaction depends on the frequency of the contact or collision. In this scenario, the complexes are able to contribute by coordinating with the reactants in a specific orientation, allowing the reaction to occur. This reduces the randomness of successful contact/collision between the reactants and thus increases the rate of reaction.

Catalysis is divided into heterogeneous and homogeneous catalysis, where the former refers to catalysts being in a different phase than that of the reactants while the latter implies that both the catalyst and the reactant exist in the same phase (usually liquid or gas) throughout the reaction. Most heterogeneous catalysts are in solid phase and adsorb reactants, be it in liquid or in gas phase on their surface for the reaction to occur. The reaction depends on how the adsorption occurs and the total surface area of the catalyst plays an important role on the reaction rate. Homogeneous catalysts, on the other hand, are generally dissolved in the same solvents together with the reactants 
and the solubility of the catalyst can affect the rate of reaction. The focus of this work, homogeneous transition metal catalysis, is one of the important fields in industrial chemistry that has contributed to a number of applications in synthetic processes. Some transition metal complexes of platinum, ${ }^{1-10}$ palladium ${ }^{11-18}$ and mercury ${ }^{19-21}$ were found to play an important role in several catalytic processes involving the functionalization of hydrocarbons.

Gold complexes, however, had been perceived to be catalytically inferior to other transition metal complexes for a very long time, owing to gold's chemical inertness. In 1985, Hutchings reported a correlation of the activity of metal chloride catalysts in the hydrochlorination of acetylene with their respective standard reduction potentials. He recognised that gold chloride with a standard electrode potential of $1.42 \mathrm{~V}$ has potential in the activation of alkynes. ${ }^{22}$

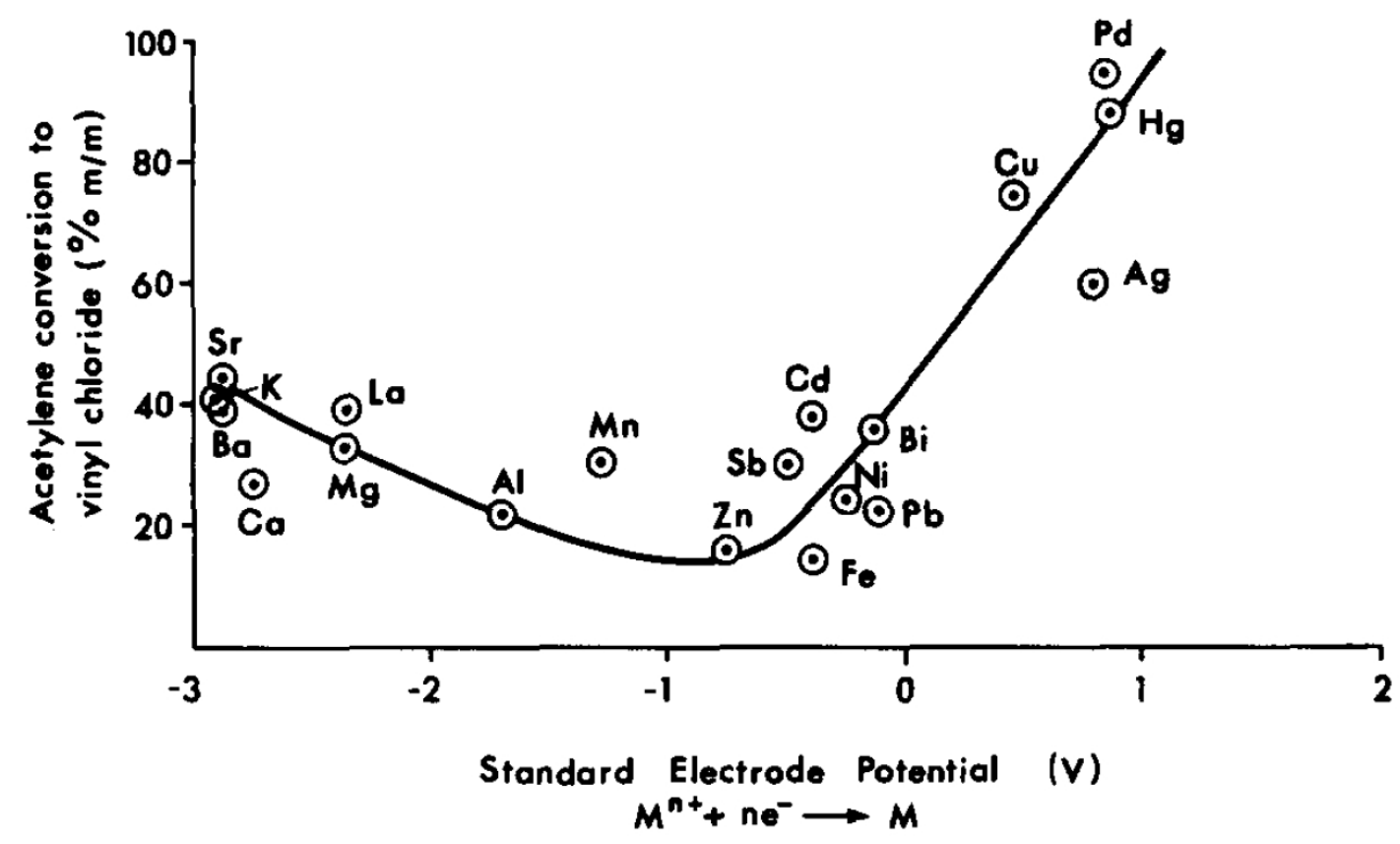

Figure 1.1 The correlation of hydrochlorination of activity of metal chlorides with the standard electrode potential. (Reproduced with permission; from Hutchings ${ }^{22}$ copyright (c) 1985 Elsevier.)

As shown in Figure 1.1, Hutchings predicted that the metal chlorides with a positive electrode potential may interact differently with acetylene and vinyl chlorides than the 
metals with an electrode potential smaller than zero. Ever since, the view of gold chemistry has changed significantly and the interest in gold catalysis has increased drastically. Some gold complexes have been demonstrated to possess catalytic properties in numerous organic reactions and these properties have been the subject of much research over the decades. ${ }^{23-44}$ Their unique electronic structure, due to relativistic effects, and their soft carbophilic nature permit the activation of unsaturated $\mathrm{C}-\mathrm{C}$ bonds towards nucleophilic addition which will be discussed in further details in the later sections.

One important reaction that received a lot of attention is the gold-catalysed nucleophilic addition of various functional groups to unsaturated hydrocarbons. ${ }^{23-36}$ It was found that complexes containing gold metal centres are able to activate $\mathrm{C}-\mathrm{C}$ multiple bonds in alkenes, alkynes and allenes for nucleophilic addition. Gold catalysts are involved in a wide range of nucleophilic addition reactions, such as hydration, ${ }^{23-27}$ hydroalkoxylation, ${ }^{25-30}$ hydroamination, ${ }^{30-33}$ cycloaddition, ${ }^{37-40}$ intramolecular nucleophilic attack and intermolecular reaction between two unsaturated hydrocarbons. ${ }^{45-48}$ In this work, the intermolecular addition of alcohols to alkynes was adopted as the main focus of the investigation. This includes the details of the mechanism undergone by gold catalysts and the important properties of the mechanism. 


\subsection{Reaction of alkynes with oxygen containing nucle- ophiles}

First, we look at the properties of alkynes which allow nucleophilic addition reactions to occur. The lowest unoccupied molecular orbital (LUMO) of alkynes is relatively low in energy and allows interaction with strong nucleophiles such as alkoxide ions. Although weak nucleophiles such as alcohols and water do not react with alkynes, this reaction is possible in the presence of a catalyst that withdraws electron density from the alkyne, making it more electrophilic. This consequently allows the attack of weak nucleophiles. ${ }^{49}$ Although the use of $\mathrm{Hg}(\mathrm{II})$ salts in an acidic medium produces good yields for the addition of alcohols to alkynes, the catalyst itself is highly toxic and requires harsh conditions for the reaction to occur. Many years later, some gold salts were then found to be competitive with the catalytic ability of $\mathrm{Hg}$ (II) salts. ${ }^{50}$ Since then, the need to develop better gold catalysts to overcome the disadvantages of $\mathrm{Hg}$ (II) catalysts has attracted chemists from around the world to venture into this particular field. Fukuda and Utimoto were the first to report on the $\mathrm{Au}(\mathrm{III})$ catalysed $\left(\mathrm{NaAuCl}_{4}\right)$ addition of alcohols and water to unactivated alkynes, forming ketals and ketones, respectively. ${ }^{26,27}$ Scheme 1.1 shows a summary of Fukuda and Utimoto's work. They also reported that when $\mathrm{NaAuCl}_{4}$ was replaced with a

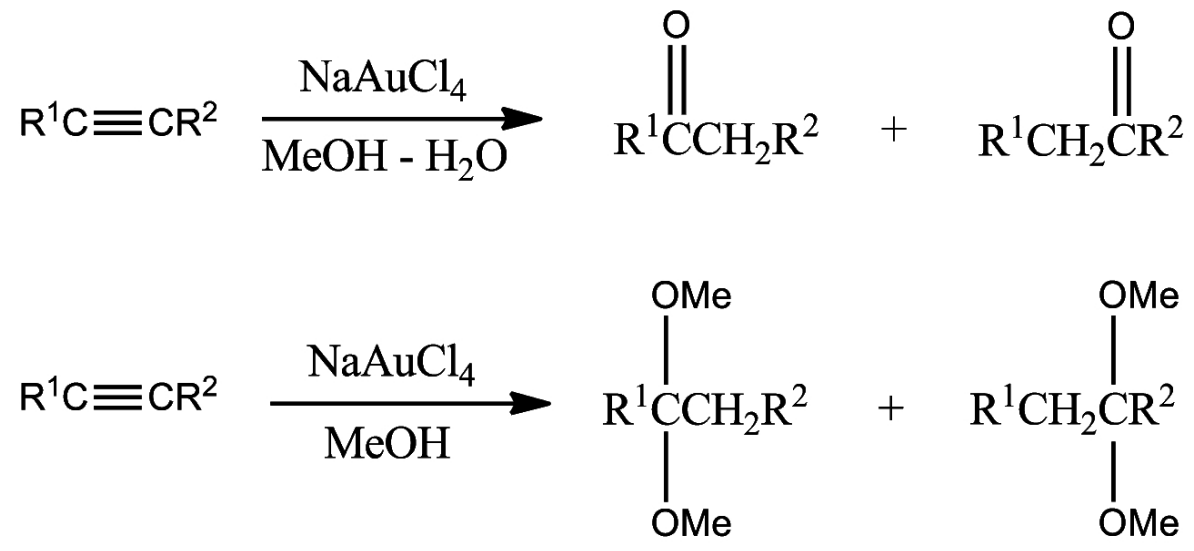

Scheme 1.1 Reaction scheme for the formation of ketones and ketals from unactivated alkynes.

$\mathrm{Au}(\mathrm{I})$ complex, $\mathrm{KAu}(\mathrm{CN})_{2}$, the alkynes were recovered unchanged. Some problems were 
reported for the catalyst under study. One of them was that the presence of a propargylic hydroxyl group in alkyne resulted in a rather sluggish reaction. The other more serious problem, which occured was the deactivation of the catalyst due to the reduction into inactive metallic gold under the reaction conditions.

In the years that followed, Teles et al. reported that cationic $\mathrm{Au}(\mathrm{I})$ complexes of the general type $\left[\mathrm{L}-\mathrm{Au}^{+}\right]$, where $\mathrm{L}$ was a phosphane, phosphite or an arsine were excellent in catalysing the nucleophilic addition reaction of alcohols and alkynes. ${ }^{28}$ These catalysts were able to achieve excellent yields with turnover frequencies of up to $5400 \mathrm{~h}^{-1}$ under mild conditions. They were also insensitive towards the presence of air or water and the reaction was able to be conducted without the involvement of a solvent. More importantly, these catalysts were able to overcome the disadvantages found with Utimoto's catalysts especially concerning the deactivation of the catalyst was concerned. Propargyl alcohols, for example, were able to react smoothly using the catalyst system reported by Teles. These reactions occurred in the presence of $\mathrm{H}^{+}$as cocatalyst. The use of cationic $\mathrm{Au}(\mathrm{I})$ systems proved to be a good choice due to the reinforced effect it has on the electronic structure of the substrate: the study showed that, in general, cationic gold systems are more electrophilic in nature compared to neutral gold catalysts and therefore, they withdraw more electrons from alkyne, causing the substrate to be more susceptible to nucleophilic attack. Alkynes with higher electron density at the triple bond are more reactive due to the increased $\pi$-basicity for coordination with the electrophilic gold catalyst. Steric hindrance, on the other hand, inversely correlates with the reactivity of alkynes. As for the reactivity of alcohols used, it was reported that the order of reactivity decreases ten fold when switching from primary alcohols to secondary. Tertiary alcohols and phenols do not react with alkynes.

The activity of gold catalysts can also be increased by changing reaction conditions and ligands attached to the gold species appropriately to give an improvement in the reaction yield. In 2002, Tanaka and coworkers reported that $\mathrm{Au}(\mathrm{I})$ complexes in aqueous methanol 
and in the presence of strong acids served as a powerful catalyst in the hydration of alkynes to their respective Markovnikov ketone products. ${ }^{23}$ They mentioned that the efficiency of the catalyst was also very much dependent on the ligands attached to the complex. While the effects of the ligands were unclear to them at that time, they predicted that the appropriate ligands could stabilise the catalyst and prevent from converting into inactive metallic particles. They also claimed that the reaction did not occur when either the Au catalyst or the acid cocatalyst was absent from the reaction. This is in good agreement with the study by Teles et. al where the $\mathrm{H}^{+}$species was used as cocatalyst. ${ }^{28}$ However, a study by Hartwig ${ }^{51}$ and $\mathrm{He}^{52}$ in the later years showed that triflic acid, $\mathrm{CF}_{3} \mathrm{SO}_{3} \mathrm{H}$ was able to catalyse the nucleophilic addition to olefins without the presence of gold complexes. This finding brought forth a study by Ujaque to compare the mechanism between acid- and gold-catalysed nucleophilic addition reactions to olefins since both species were discovered to catalyse the reaction independently. ${ }^{30}$ By means of a theoretical approach, they explored the mechanism of $\mathrm{Me}_{3} \mathrm{Au}\left(\mathrm{CF}_{3} \mathrm{SO}_{3}\right)$, gold(I)- and $\mathrm{CF}_{3} \mathrm{SO}_{3} \mathrm{H}$, triflic acid-catalysed addition reaction of $\mathrm{O}$ - and $\mathrm{N}$-nucleophiles to ethene. The results showed that gold catalysts performed better in hydroamination reaction while the acid-catalysed reaction was more favorable in hydroalkoxylation. However, none of the catalysts under study showed any distinct advantage over one another.

Although the use of strong acid as cocatalyst has provided good conversion and yield of the reaction, a large quantity of acid is required and is rather difficult to handle. As an alternative, silver salts were used for extraction of chloro ligands from gold catalysts to produce an active catalytic species. ${ }^{24,25,53}$ However, the drawbacks of using silver salts to activate the gold complex in the reaction include sensitivity of silver compounds towards light, the high price of silver salts and the possibility that silver can be a catalyst itself in the reaction, which would affect the reaction profile of the otherwise gold-only catalysed reaction. Such ambiguity in the reaction makes the mechanistic investigation of the reaction much harder than it is otherwise. In order to avoid this problem, Corma and coworkers introduced an acid-free and silver-free gold catalysed alkyne hydration 
procedure with the use of Gagosz-type ${ }^{54,55}$ complexes. ${ }^{56}$ This procedure requires milder reaction conditions but comes at the expense of the amount of catalyst required. It also takes a longer time for the reaction to complete. In a recent paper, Nolan and coworkers managed to synthesise a digold complex that yielded $95 \%$ conversion in hydration of alkyne with only $0.05 \%$ of catalyst. ${ }^{57}$

\subsection{Nucleophilic addition of unsaturated hydrocarbons}

Gold catalysts were found to be effective not just for the activation of alkynes but also for several other types of unsaturated hydrocarbons such as alkenes and allenes towards nucleophilic attack. When dealing with unsaturated hydrocarbons, researchers often have to deal with product mixtures if the reactants possess multiple reaction sites suitable for nucleophilic attack. This selectivity problem is mostly resolved by using protecting groups in the substrate to deactivate unwanted active sites or taking advantage of bulky substituents to block the incoming nucleophilic attack at an unwanted reaction site of the substrate. In a catalytic reaction, the catalyst can also take responsibility for guiding the substrates in the right orientation for the reaction to occur. There are different selectivity problems that have to be dealt with when handling different unsaturated hydrocarbons. The selectivity problems that are commonly seen are regioselectivity (Markovnikov against anti-Markovnikov orientation) and stereoselectivity (cis- or trans-isomers) when alkenes are involved in the reaction. As the number of unsaturated bonds increases in the substrate, the selectivity problem becomes more complicated. When dealing with alkynes, the problem with chemoselectivity (single or double addition to the substrate) in addition to regio- and stereoselectivity arise. As for allenes, positional selectivity adds to the variety of products that can be formed depending on which of the two orthogonal double bonds that will react in the case of single addition. Figure 1.2 summarises the selectivity options in unsaturated hydrocarbons with the arrows indicating the possible 
positions for nucleophilic attack. Having said that, there are three major principles that have been highlighted in Hashmi's review to direct these selectivity alternatives: ${ }^{58}$ 1) The positional selectivity was often controlled by steric hindrance by substituents on only one side of the double bonds in intermolecular reactions, 2) In intramolecular reactions, problems with positional selectivity can be solved by placing the groups at a distance such that a five- or six-membered ring product will be formed and lastly, 3) functional groups on the carbon atom next to the allene unit allowed the control of the selectivity by geometrical and electronic restrictions in both inter- and intramolecular reactions.

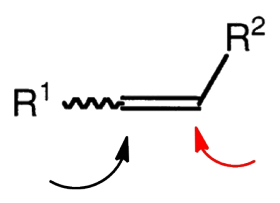

regioselectivity<smiles>[R]C(CC)=C(CC)CC</smiles>

stereoselectivity

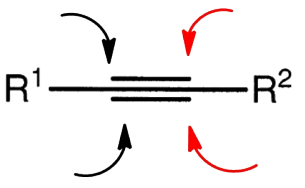

regioselectivity

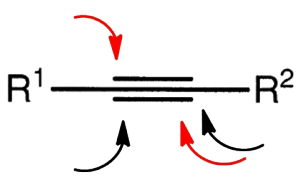

stereoselectivity

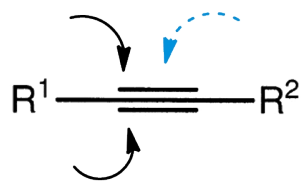

chemoselectivity

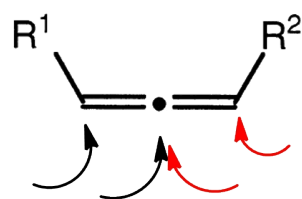

regioselectivity

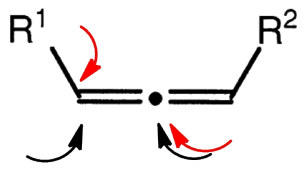

stereoselectivity

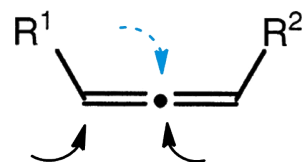

chemoselectivity

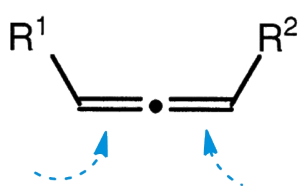

positional selectivity

Figure 1.2 Selectivity problems in alkenes, alkynes and allenes. The black and red arrows represent the two possibilities of nucleophile attack at different positions while the blue arrows represent single nucleophile addition in alkynes and allenes. 
Most of the nucleophilic additions to allenes are intramolecular and this provides a good synthetic route for producing heterocycles such as pyrans and furans. In such reactions, allenes are activated through coordination with gold catalysts $(\mathrm{Au}(\mathrm{I})$ or $\mathrm{Au}(\mathrm{III}))$ at one of the peripheral double bonds. Similar to alkynes, the multiple bonds in allenes become more electrophilic and susceptible towards nucleophilic attack. An alternative pathway was also reported if an $\mathrm{Au}(\mathrm{III})$ complex is used as the catalyst. ${ }^{59}$ After coordinating with the substrate, the $\mathrm{Au}(\mathrm{III})$ catalyst is being reduced to an $\mathrm{Au}(\mathrm{I})$ species to activate the multiple bonds for the upcoming nucleophilic attack. The formation of an activated complex in the transformation of allenes appears to have sparked different opinions in this field. Two possible intermediates were proposed in the reaction mechanism, which were the $\eta^{2}$-allene gold complexes and gold-stabilised allylic cations as shown in Scheme 1.2. The latter may be formed in a planar conformation if sterically allowed and this would cause the loss of stereochemical information carried by the starting allene. It should be noted that in the presence of both alkyne and allene moieties in a molecule, the gold complex activates the triple bonds on the alkyne towards intramolecular nucleophilic attack by the allene. However, in the presence of water, the gold catalyst selectively activates the allene group and triggers the intramolecular nucleophilic attack of alkyne onto allene. ${ }^{60-62}$ Many gold-catalysed nucleophilic reactions have been studied using allene as one of the reactants and the studies proved that allene can be easily activated towards the attack of a wide range of nucleophiles. ${ }^{34,36,42,63,64}$

$[\mathrm{Au}]$

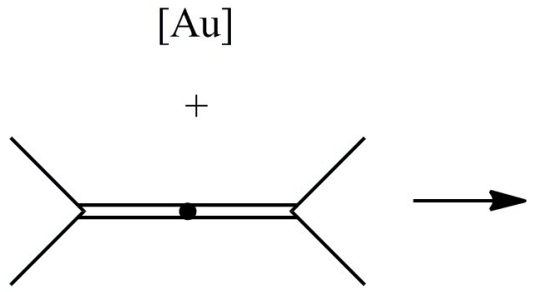

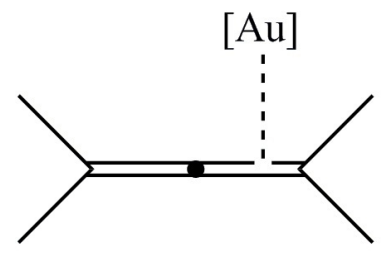

I

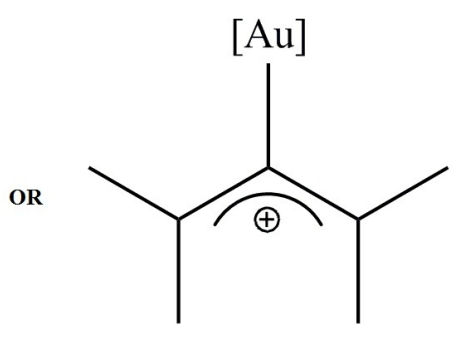

II

Scheme 1.2 The possible intermediates proposed in the reaction mechanism involving allenes. $\eta^{2}$-complex (I) and carbocation (II) intermediates. 
Nucleophilic reactions involving alkenes have also been extensively studied. ${ }^{42}$ The nucleophilic attack on alkenes is governed by only regioselectivity and stereoselectivity. Judging from the selectivity problems associated with alkenes, we would expect that alkenes, having the least selectivity problems would attract more attention compared to the other unsaturated hydrocarbons. However, this is not the case in earlier studies. Several studies and reviews in the past showed that alkenes are less likely to be catalysed by gold complexes. ${ }^{65}$ This may be due to the fact that the highest occupied molecular orbitals (HOMOs) and LUMOs in Au-alkene activated complexes are energetically higher than $\mathrm{Au}$-alkyne complexes, making them less electrophilic for nucleophilic attack. ${ }^{66}$ In order for alkenes to be activated by gold catalysts in a nucleophilic addition, the catalyst has to be stabilised by another species in the reaction. In a recent report, Zhang et al. discovered that the nucleophilic addition of alkenes, in particular, can be made possible with the activation by $\mathrm{Au}(\mathrm{III})$ or $\mathrm{Pd}(\mathrm{II})$ catalysts stabilised by $\mathrm{CuCl}_{2} \cdot{ }^{67-69}$ The role of $\mathrm{CuCl}_{2}$ was to stabilise the $\mathrm{Au}(\mathrm{III})$ and $\mathrm{Pd}(\mathrm{II})$ by retarding the reduction of $\mathrm{Au}(\mathrm{III})$ and $\mathrm{Pd}(\mathrm{II})$ salts and/or oxidizing the metal gold and palladium back to their higher oxidation states. They tested the stabilised catalysts with the addition of alcohols, amines and phenols to unactivated alkenes and the alkenes were reported to be active in these reactions. Such improvement on the catalytic system offers a huge possibility to synthetic processes involving the formation of heterobonds in alkene substrates and also the formation of heterocyclic compounds.

\subsection{Catalytic system}

The improvement on a catalytic reaction is often discovered by adjusting the structure of the catalyst itself that plays an important role in the reaction. The basic role of a catalyst is to lower the activation barrier of the reaction, thus providing an alternative pathway to the one without the presence of a catalyst. In addition, catalysts are also able to change the 
electronic structure of a substrate and affect the spatial arrangement around the reactants which could have a positive effect on the reaction. These effects can be adjusted by changing the structure of the catalyst to improve the selectivity, speed and yield of the reaction.

One of the many ways to alter the activity of an organometallic catalyst is to change the type of ligands attached to the metal centre. By changing the ligands attached to the metal centre, one can alter the electronic structure of the catalyst and thus, affect its efficacy as a catalyst. The effects of different types of ligands on gold catalysts have been the topic of much research. Teles and coworkers ${ }^{28}$ tested a number of phosphorus containing ligands and the order of activity of the ligands was as follows: $\mathrm{Ph}_{3} \mathrm{As}<$ $\mathrm{Et}_{3} \mathrm{P}<\mathrm{Ph}_{3} \mathrm{P}<\left(4-\mathrm{F}-\mathrm{C}_{6} \mathrm{H}_{4}\right)_{3} \mathrm{P}<(\mathrm{MeO})_{3} \mathrm{P}<(\mathrm{PhO})_{3} \mathrm{P}$. This investigation showed that the electronic properties of the ligands directly influence the capability of gold complexes as catalysts. In hydroalkoxylation or hydration of alkynes, high electrophilicity of the gold catalyst is desired to obtain high reactivity, ergo low Lewis basicity of ligands is highly desirable. Although, electron-poor ligands in this study increase the activity of the catalyst, the stability of the complex decreases. The catalyst with the $(\mathrm{PhO})_{3} \mathrm{P}$ ligand was more than twice as active as the catalyst with the $\mathrm{Ph}_{3} \mathrm{P}$ ligand but the former deactivated after only 2500 turnovers while the latter was still active after 5000 turnovers. A balance between the stability and activity has to be achieved in order to obtain a good catalyst for such reactions. It can be seen that gold complexes with P-containing ligands are good catalysts for the hydration or hydroalkoxylation of alkynes. ${ }^{23,28,70}$ The catalytic ability of such complexes was investigated by Khin et al. by studying the mode of catalyst deactivation in this reaction. ${ }^{53}$ The gold(I) complex with $\mathrm{PPh}_{2} \mathrm{Py}$ ligand was found to deposit a gold mirror on the glass surface in many of the organic reactions where it was used as catalyst. In certain cases, a red crystalline aggregate was found after the reaction. This aggregate was analysed using single X-ray crystallography and it was shown that the deactivation was caused by the formation of a unique trinuclear gold(I) aggregate as shown in Figure 1.3. As well as forming gold(I) trimer aggregates, the deactivation was 
also reported to occur in solution when the complex coordinates with an adjacent complex through the nitrogen atom in the ligand.

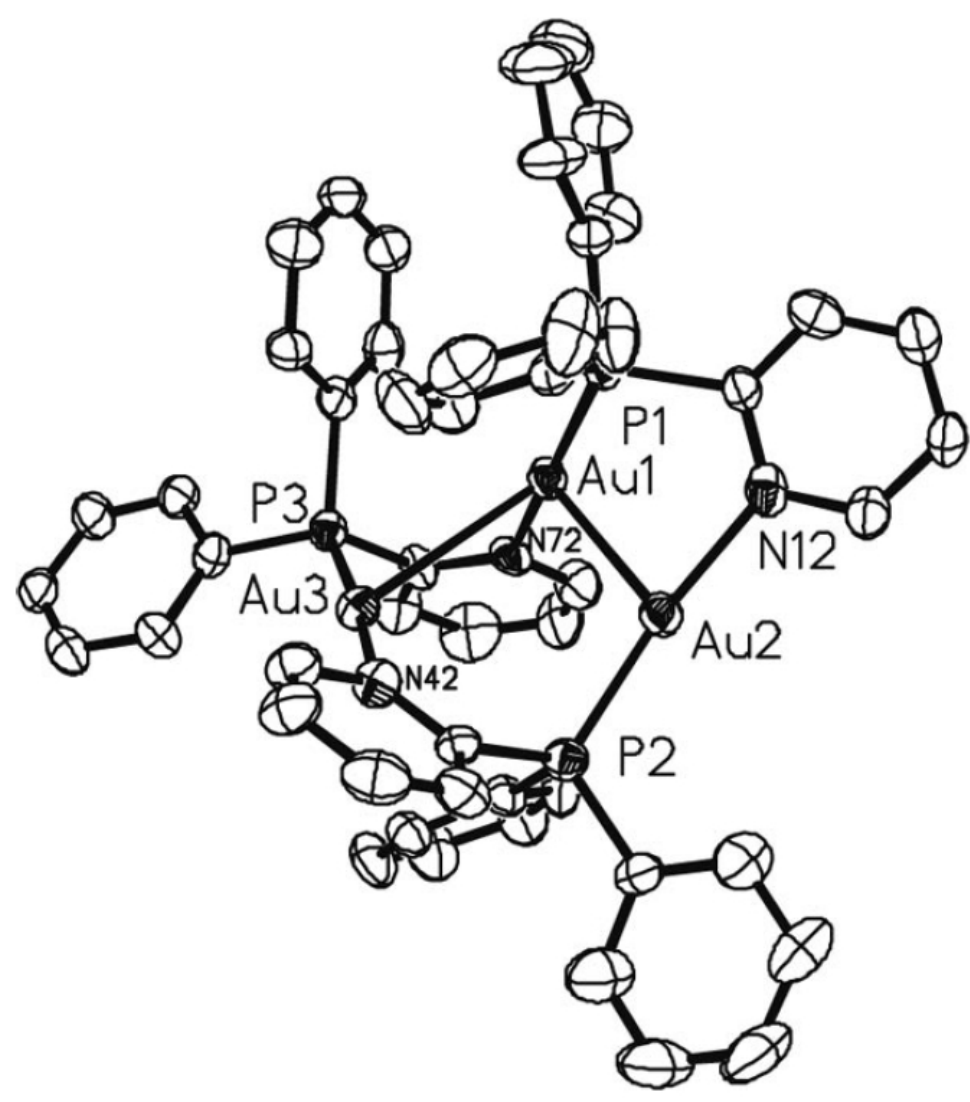

Figure 1.3 The deactivated gold(I) trimer observed by Khin et al. (Reproduced with permission; from Khin et al. ${ }^{53}$ copyright (c) 2010 Wiley.)

The lifetime of a catalyst has always been the main issue in catalytic reactions. A successful catalyst is normally perceived to have a high turnover number in reactions and is only required in a small amount in the reaction i.e. low catalyst loading. The basic approach to increasing the lifetime of gold catalysts is to reduce the concentration of $\mathrm{Au}$ species in the reaction as it decomposes at a rate proportional to the square of its concentration. However by doing that, the reaction gets retarded and this may lead to incomplete reaction. The need for research to extend the lifetime of gold catalysts became very important for future development. Much research has been done to extend the lifetime of gold catalysts using different approaches, which include adding another 
metal complex in the reaction to stabilise the gold catalyst. Graf et al. studied the lifetime of $\mathrm{AuCl}_{3}$ in a three-component coupling reaction between piperidine, phenylacetylene and benzaldehyde. ${ }^{71}$ It was reported that the catalyst achieved the best catalytic ability when 2,2,6,6,-tetramethylpiperidine 1-oxyl (TEMPO) and copper(II) chloride, $\mathrm{CuCl}_{2}$ were added in the reaction. On a side note, the reaction did not show any conversion if the gold catalyst was absent from the reaction, indicating that both $\mathrm{CuCl}_{2}$ and TEMPO did not catalyse the reaction.

Gold salts can be reduced to inactive metallic gold particles by alkynes, alkenes, alcohols, $\mathrm{CO}$ and phosphines present in the reaction medium, which renders the catalyst useless in the reaction. ${ }^{26-28,45,72}$ Therefore, stability of the catalysts has to be ensured for the catalytic reaction to proceed efficiently. Tanaka and coworkers ${ }^{23}$ predicted that the ligands (in this case, triphenyl phosphite) stabilise the catalyst from decomposition and this was proven to be true by Nolan's research ${ }^{43}$ on the catalytic rearrangement of allylic acetates using a N-heterocyclic carbene (NHC) gold system. It was found that bulky ligands were able to shield the gold centre more effectively, thus preventing decomposition of the metal centre. NHC gold complexes have been studied in a wide range of organic transformations and have contributed a lot to the improvement of gold catalysis. ${ }^{73}$ NHCbased ligands have managed to increase the performance of gold catalysts due to a number of factors the ligands can contribute to the complex. They have good $\sigma$-donor properties that increase the strength of the metal-carbene bond. In catalysis, this means that a higher thermal stability can be achieved and subsequently enables the catalyst to survive in harsher reaction conditions. ${ }^{74,75}$ By changing the substituents on nitrogen atoms and the nature of the heterocyclic ring itself, NHCs offer the freedom to tune the electronic and steric properties of the metal centre. ${ }^{76-78}$ Furthermore, the solubility of gold complexes in aqueous or organic media can also be enhanced with different derivatives of NHC ligands. The possibility of tuning the solubility of the complex in certain solvents especially in aqueous medium enables some gold-catalysed organic transformation to be more efficient. ${ }^{79,80}$ For example, alkynes have low solubility in water and by coordinating 
with a more water soluble gold complex, the hydration reaction can proceed at a higher rate.

In 2003, Laguna and coworkers investigated the catalytic ability of different organometallic $\mathrm{Au}(\mathrm{III})$ compounds to activate terminal alkynes to the addition of water and methanol. ${ }^{25}$ They found that at least one chloro ligand was essential for the reaction to progress whereas a weakly coordinating neutral ligand was able to inhibit the catalytic process. Speculations have been made in the past that gold catalysts were only able to catalyse the reactions due to the active $\mathrm{Au}(\mathrm{I})$ species but Laguna and coworkers proved that $\mathrm{Au}(\mathrm{III})$ catalysts in their study did not reduce to $\mathrm{Au}(\mathrm{I})$ species in these reactions as there was no $\mathrm{Au}(\mathrm{I})$ intermediate identified by spectroscopic methods. Therefore, they predicted that the reaction is in fact activated by $\mathrm{Au}(\mathrm{III})$ species in the form of $\mathrm{AuCl}_{3}, \mathrm{Au}\left(\mathrm{C}_{6} \mathrm{H}_{5}\right) \mathrm{Cl}_{2}$ or $\mathrm{Au}\left(\mathrm{C}_{6} \mathrm{H}_{5}\right)_{2} \mathrm{Cl}$. It is also worthy to note that the addition of an acid cocatalyst was found to improve the activity, perhaps implying that the acid cocatalyst prevented the decomposition of the gold catalyst into inactive metallic gold. Further investigation by Schmidbaur and coworkers showed that using $\mathrm{BF}_{3} \cdot \mathrm{OEt}_{2}$ as acidic promoter for $\mathrm{Au}(\mathrm{I})$ catalysts ensured a high turnover frequency up to $3900 \mathrm{~h}^{-1} \cdot{ }^{70}$

As well as changing the electronic structure of the catalyst, ligands play an important role in the formation of intermediates along the reaction pathway. Researchers believed that the electrophilic activation of the triple bond in alkynes will produce either gold carbene or carbocations as the key intermediate in the reaction as shown in Scheme 1.3. For example, in studies of gold-catalysed enyne cycloisomerisation, ${ }^{81-83}$ the formation of gold carbene species was suggested based on mechanistic studies using deuterium labelling while results from trapping experiments, NMR studies and substituent effects on regioselectivity supported a carbocationic species to be the key intermediate. ${ }^{84-86}$ However, little information was reported to support either of these predictions. A few years later, Fürstner proposed that the intermediate in the reaction can be best represented by either cationic or carbenoid resonance extremes depending on the metal 
template. ${ }^{87}$ The debate on the presence of which of the two intermediates in the reaction mechanism went on for a few years. Using a theoretical approach, Toste and coworkers ${ }^{88}$ were able to lay this controversy to rest by determining barriers to bond rotation in carbenoid/carbocationic species and activation energy of cyclopropanation reactions. They came to the conclusion that the formation of intermediate species can be at any point in a range of being a singlet carbene to a metal-coordinated carbocation, depending on substituents on the substrate and the ligands on the metal. An electron withdrawing ligand further diminishes the electron density on the metal centre, weakening the $\pi$-back donation to the carbon atom on the substrate and thus will produce the carbocation intermediate. On the other hand, an electron donating ligand increases the electron density on the metal centre, allowing more facile $\pi$-back donation to the substrate, thus leading to the formation of carbene intermediates.

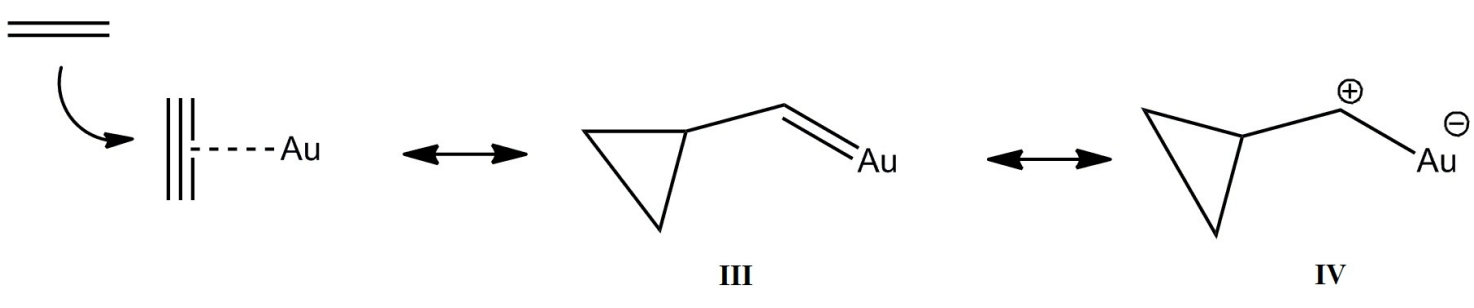

Scheme 1.3 The possible intermediates proposed in the reaction mechanism involving alkynes. Carbene (III) and carbocation (IV) intermediates.

To probe a deeper understanding in the nature of intermediates in gold-catalysed hydration reaction, Velegraki et al. devised an experiment to investigate the interaction between the gold catalyst and alkynes substrate by using acetylenes containing aryl-substituted cyclopropyl (as shown in Scheme 1.4). ${ }^{89}$ As depicted in Scheme 1.4, there exist three possibilites in which the interaction can occur; a loosely coordinated gold complex (symmetrical $\mathbf{V}$ and asymmetrical VI) and a vinyl carbocation VII. If the interaction is based on that of the vinyl carbocation, the allene intermediate would be formed and detected as shown in Scheme 1.5. The results of the experiment showed that both gold(I) and gold(III) catalysts interact with alkynes through the formation of loosely bound metal- $\pi$ complex as the allene-type side products were not detected. 


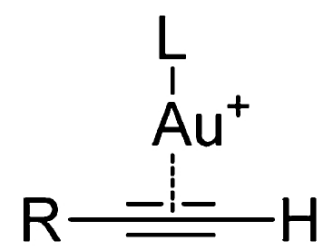

$\mathbf{V}$

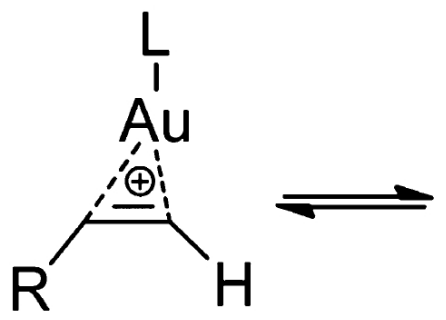

VI

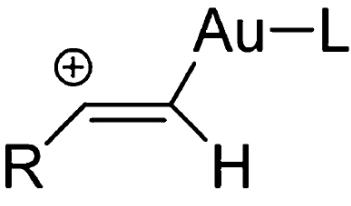

VII

Scheme 1.4 Possible interaction between gold(I) catalyst and terminal alkyne. (Reproduced with permission; from Velegraki and Stratakis ${ }^{89}$ copyright (C)2013 American Chemical Society.)
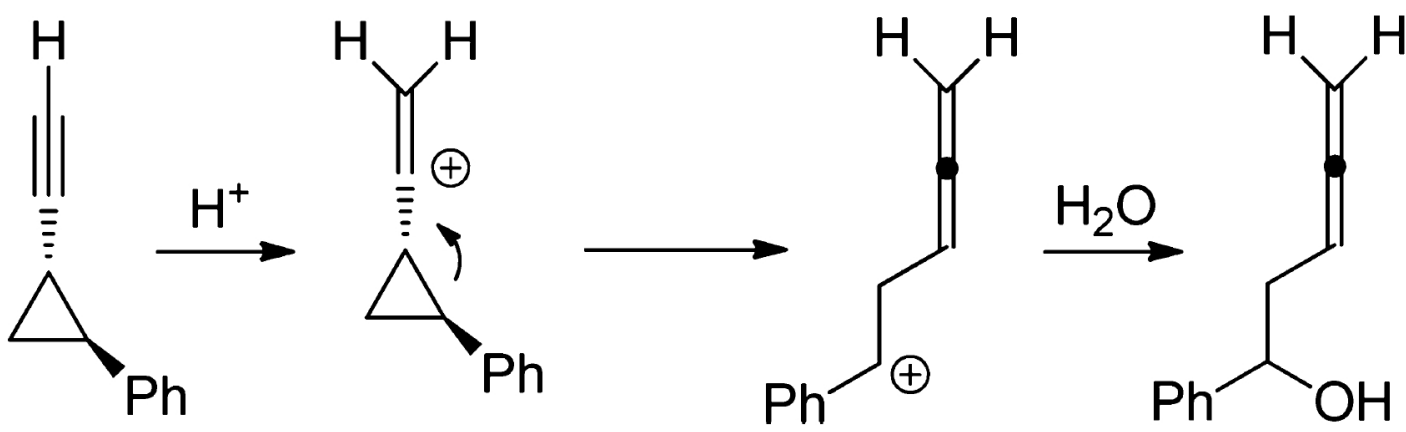

Scheme 1.5 Rearrangment of vinyl carbocation to form an allene structure. (Reproduced with permission; from Velegraki and Stratakis ${ }^{89}$ copyright (C) 2013 American Chemical Society.)

Different gold complexes have been studied to control the selectivity in the transformation of unsaturated hydrocarbons. ${ }^{90-93}$ Different ligands were employed and the effects they have on the selectivity control were investigated. As an example, a study by Corma et al. investigated the regio- and stereoselectivity of gold-catalysed intermolecular hydroalkoxylation of alkynes. ${ }^{91}$ Alkynes normally prefer to react in a two-fold addition of alcohols to form ketals instead of vinyl ethers which are produced from the monoaddition reaction. In addition to that, the stereoselectivity in internal alkynes further complicates the process to afford the desired vinyl ethers. Corma et al. reported that complexes with electron-withdrawing ligands result in a nearly complete selectivity towards the monoaddition product while electron-donating ligands lead to the formation of ketals. It is also worthy of note that the high selectivity achieved by these catalysts did not come from kinetic control. Enantioselectivity control in a reaction can also be adjusted with the use of the appropriate chiral ligands to afford the desired product. ${ }^{94}$ In some cases, 
efficient chirality transfer can also be achieved through a chiral counterion even though the catalyst itself is achiral, as first demonstrated by Toste and coworkers. ${ }^{95}$

$\mathrm{Au}(\mathrm{I})$ complexes are effective catalysts because they have the lowest possible coordination number, lower than that of $\mathrm{Pt}(\mathrm{II})$ or $\mathrm{Pd}(\mathrm{II})$ which allows the substrate easy access to the metal centre. This explains the effectiveness of Teles's catalysts with general type $\left[\mathrm{L}-\mathrm{Au}^{+}\right]$in activating alkynes to nucleophilic addition. The electrophilic nature of such a complex is only governed by one ligand and can be easily modified by changing the steric and electronic properties of the ligand and by the choice of counterion and solvent. However, having a low coordination number also means that the ligand must be ready to leave the metal centre to provide a coordination site for the activation of $\mathrm{C}-\mathrm{C}$ multiple bonds.

\subsection{Comparison between gold and other transition metal catalysts}

A catalysed nucleophilic addition to alkynes is not a new breakthrough in organic synthesis. Mercury was one of the earliest catalysts involved in the different transformation of unsaturated hydrocarbons. Due to the toxicity and highly demanding reaction conditions for $\mathrm{Hg}$-catalysed reactions, researchers began to search for other alternatives. Platinum and gold catalysts offer an exceptional capability in catalysing the hydroalkoxylation and hydration of alkynes in milder reaction conditions compared to mercury catalysts. ${ }^{50}$ These metals behave in a similar manner due to the significant relativistic effects in this part of the periodic table. The physical state of mercury is a good example of a relativistic effect. It is the only liquid metal at room temperature due to the stabilisation of the $6 s$ orbitals that results in a weak $\mathrm{Hg}-\mathrm{Hg}$ interactions, causing even monoatomic $\mathrm{Hg}$ to be volatile. ${ }^{96}$ Relativistic effects in the metals will be discussed in more detail in the next section. 
The activity of the lighter analogues of gold complexes in Group 11 has been known to be lower compared to gold, mercury and platinum catalysts. This may be due to the larger relativistic effects of the heavier metal. Information regarding copper and silver catalysts is scarce and very little work has been done to investigate the catalytic ability of these complexes. However, Pouy et al. has successfully catalysed the intramolecular hydroalkoxylation and hydroamination of alkynes using $\mathrm{Cu}(\mathrm{I})$ complexes which are stabilised by NHC ligands. ${ }^{97}$ With the low cost of copper and its tolerance towards reactive functional groups, copper complexes can potentially replace the heavier metal complexes as catalysts if its catalytic performance is significantly enhanced. Silver salts were normally used as cocatalysts in gold-catalysed reactions as they extract chlorides from gold complexes to produce the active catalysts. One wonders if silver could have catalysed the reaction itself. In some cases, control experiments using silver salts in the absence of gold catalyst showed that there was no product formed. ${ }^{93}$ However with higher catalyst loading and the correct counteranion, it was reported that silver complexes were able to catalyse the hydration of terminal alkynes in mild conditions. ${ }^{98}$ It is also worth noting that internal alkynes do not react with these catalysts and thus, improving the selectivity towards terminal triple bond activation. Gold catalysts are still superior as only a small amount of catalyst is required and they are reactive enough to even activate an internal triple bond of alkynes.

Another metal complex which has profound significance in this field is a $\mathrm{Ru}(\mathrm{II})$ complex. Most metal complexes catalyse the hydration or hydroalkoxylation of terminal alkynes to form their corresponding Markovnikov products but $\mathrm{Ru}(\mathrm{II})$ complexes catalyse the reaction to form the anti-Markovnikov products. ${ }^{99-101}$ It was first discovered by Tokunaga and coworkers ${ }^{99}$ and the proposed mechanism is as shown in Scheme 1.6. The key step of this reaction pathway is the tautomerisation of 1-alkyne to form the vinylidene complex that leads to nucleophilic attack at the terminal carbon atom. This mechanism provides a good synthetic route in producing aldehydes from hydration of terminal alkynes. 


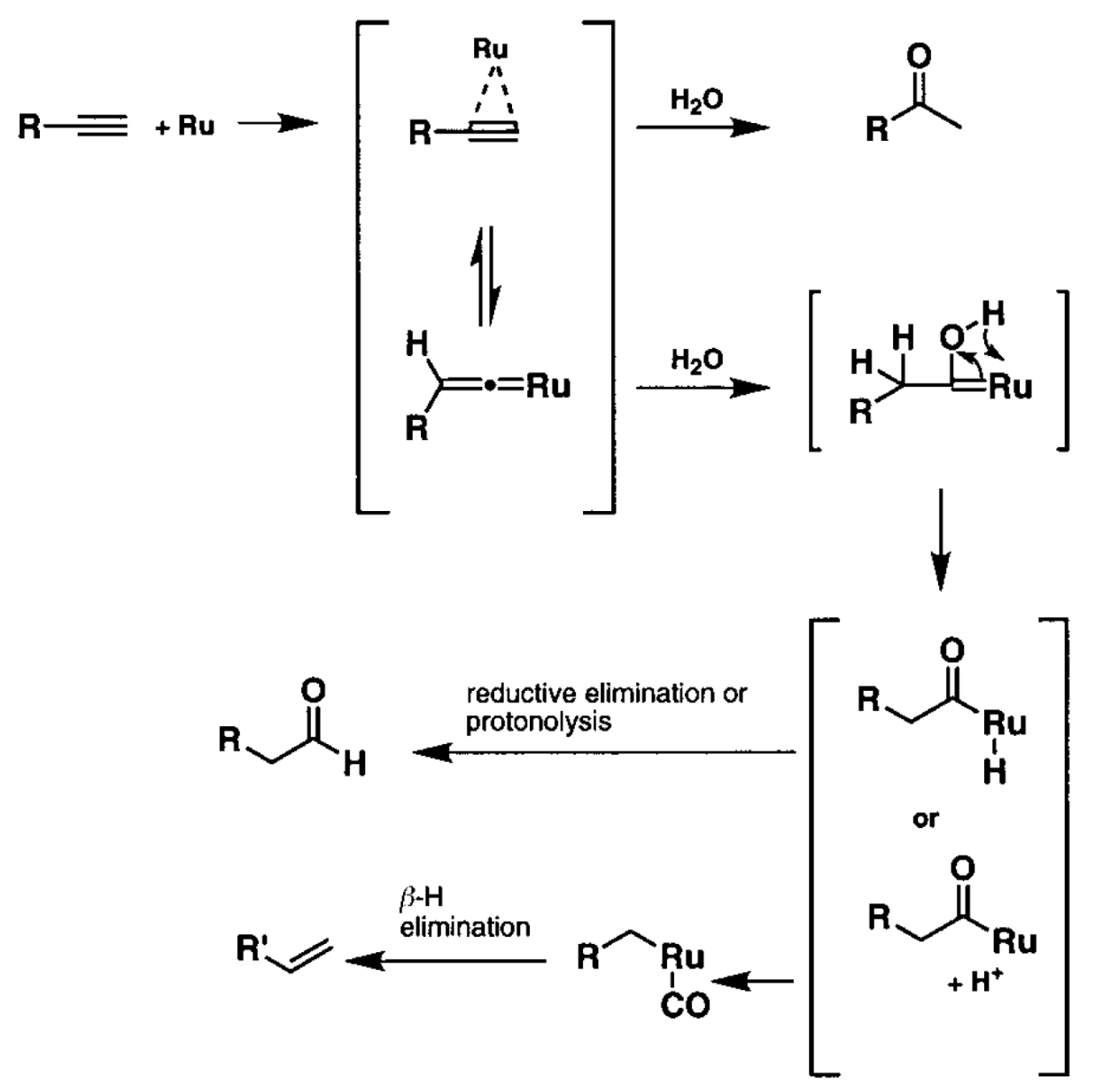

Scheme 1.6 Suggested reaction pathway in the formation of anti-Markovnikov hydration product. (Reproduced with permission; from Tokunaga and Wakatsuki ${ }^{99}$ copyright (C)1998 Wiley.)

When comparisons are made among the heavier metal complexes, the general order of reactivity of the catalysts is $\mathrm{Au}(\mathrm{I})>\mathrm{Hg}(\mathrm{II})>\mathrm{Pt}(\mathrm{II})>\mathrm{Au}(\mathrm{III})>\mathrm{Pt}(\mathrm{IV})$. Alkynes are $\pi$-donors that can be activated by $\pi$-Lewis acids such as $\mathrm{Pt}, \mathrm{Au}$ and $\mathrm{Hg}$. The reason for the higher activity exhibited by $\mathrm{Au}(\mathrm{I})$ and $\mathrm{Hg}(\mathrm{II})$ is due to the structures of metal complexes as shown in Figure 1.4. ${ }^{50} \mathrm{Au}(\mathrm{I})$ and $\mathrm{Hg}(\mathrm{II})$ are $d^{10}$ species, they form 14-electron linear structures. For the same reason, $\mathrm{Au}(\mathrm{I})$ and $\mathrm{Hg}(\mathrm{II})$ are not easily reduced to the inactive metal atoms. However, it might be worth noting that less hindered oxygen nucleophiles might be able to enter the outer sphere of these metals and reduce them to their elemental state. The difference between $\mathrm{Au}(\mathrm{I})$ and $\mathrm{Hg}(\mathrm{II})$ is that $\mathrm{Au}(\mathrm{I})$ complexes 
are able to accommodate an extra ligand if there is significant $\pi$-back donation from the filled $5 d$ orbitals to the ligands. Pt(II) and Au(III), having $d^{8}$ configuration, form a 16-electron square-planar structure which can easily provide an extra coordination site to a new molecule to give an 18 -electron species. This may not be such a good thing as it opens up a new pathway for the decomposition of the catalyst. ${ }^{50}$ Therefore, it is important that the ligands are good stabilisers to prevent the catalyst from going down the decomposition pathway. Pt(IV), a $d^{6}$ species needs more ligands to achieve a stable 18-electron configuration and therefore forming an octahedral structure. This saturated structure can hardly activate any unsaturated molecule to nucleophilic attack since the release of one ligand is not favourable. Weakly coordinated ligands have been known to increase the catalytic ability of $\mathrm{Au}(\mathrm{I})$ and $\mathrm{Hg}(\mathrm{II})$ catalysts as the ligand creates a free coordination site when it leaves the catalyst and therefore allowing the substrate to enter the coordination site.
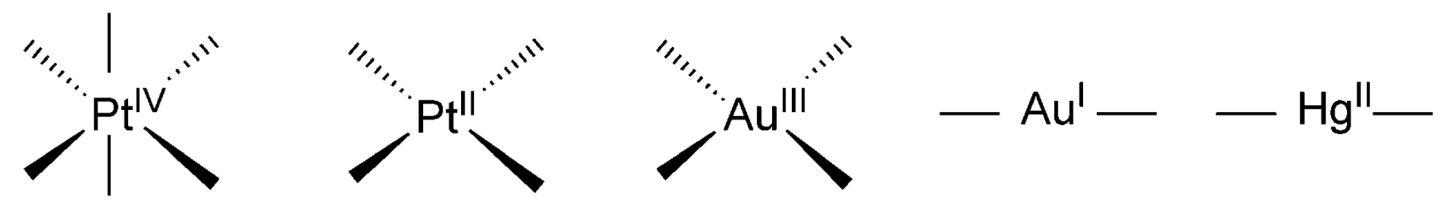

Figure 1.4 Structures of $\mathrm{Pt}, \mathrm{Au}$ and $\mathrm{Hg}$ complexes. (Reproduced with permission; from Leyva-Pérez and Corma ${ }^{50}$ copyright (C) 2012 Wiley.)

Gold and platinum complexes have superior catalytic performance compared to other metal complexes and they are commonly used in the transformation of unsaturated hydrocarbons. Several experimental results showed that gold catalysts performed better than their platinum counterparts. ${ }^{50}$ Gold catalysts were able to provide a higher yield, better selectivity and milder reaction condition without the need to exclude water and air. Relativistic effects are without a doubt one of the reasons gold performs better than platinum as a catalyst. ${ }^{102}$ In order to get more insight on the superiority of gold catalysts over platinum catalysts, Pernpointer studied the electronic structure of gold catalyst, $\mathrm{AuCl}_{3}$ and platinum catalyst, $\mathrm{PtCl}_{2}\left(\mathrm{H}_{2} \mathrm{O}\right)$ using a theoretical approach. Fully 
relativistic ab initio calculations of alkyne-catalyst complexes at Dirac-Hartree-Fock self consistent field, density functional theory and Green's function level were adopted in his study in order to account for the large relativistic effect of gold and platinum. In this study, it was reported that the Au-propyne complex had a negative LUMO energy, indicating that the complex can stabilise an excess electron from the nucleophile. In other words, Au-propyne complexes have a high affinity towards nucleophiles. On the other hand, Pt-propyne complex did not possess a negative LUMO energy. Further analysis of the pictorial representations of the HOMO and LUMO orbital densities in each complex were reported.

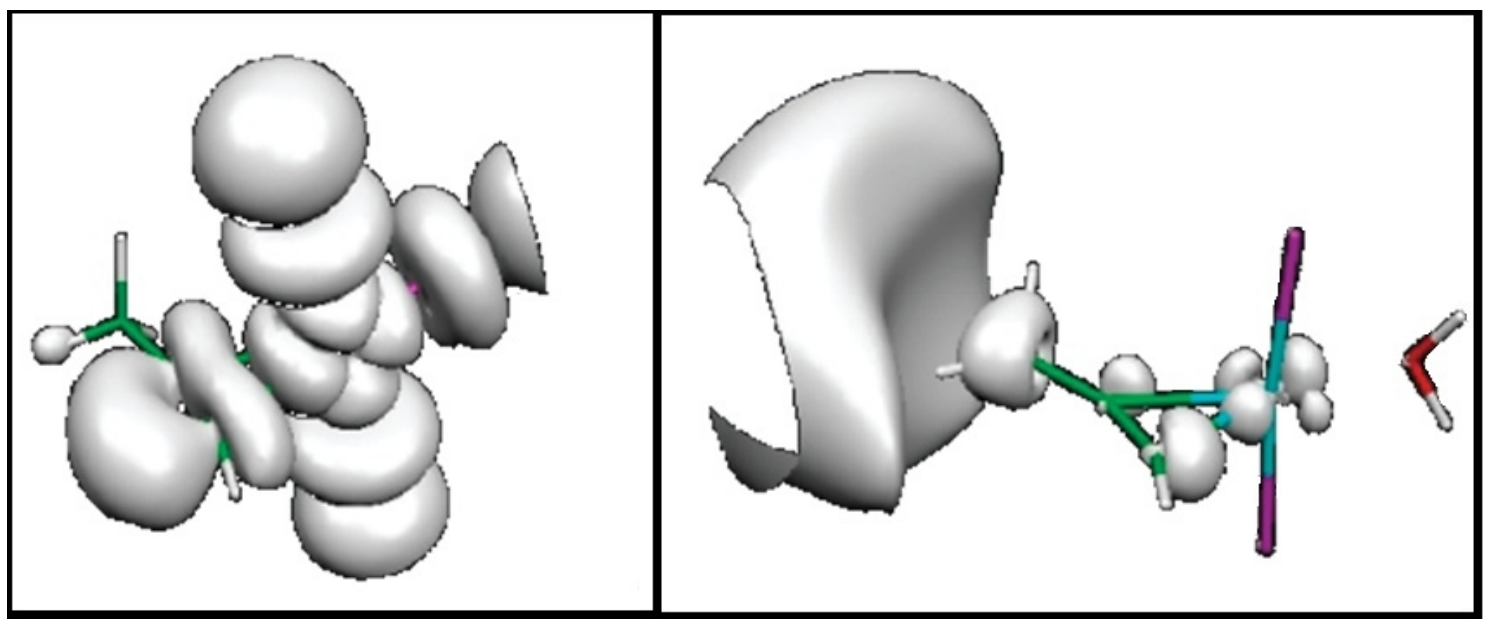

Figure 1.5 The LUMO density of Au(left) and Pt(right) complex. (Reproduced with permission; from Pernpointner and Hashmi ${ }^{102}$ copyright (C)2009 American Chemical Society.)

The HOMO orbital densities of Au and Pt complex were very similar whereas the LUMO orbital densities of both complexes showed intriguing differences which could explain the difference in catalytic ability between the two complexes. Figure 1.5 shows that Au-propyne complex possess LUMO density at the carbyne centre whereas Pt-propyne complex does not show this property. The nonexistent LUMO density around the carbyne centre implies that Pt complexes do not have a good overlap with the nucleophile frontier orbital. This finding is consistent with the results reported by Hashmi and coworkers in a similar work. ${ }^{103}$ In addition to having a negative LUMO energy, Au complexes have an advantageous distribution of the LUMO density which allows good overlap 
with the nucleophile frontier orbital. It is also worth noting that the Mulliken charge of both complexes showed that the central carbon of propyne always bears the highest positive charge, indicating that it favoured the Markovnikov addition regardless of the availability of LUMO densities. On a side note, this study displayed the advantages of using theoretical methods for predicting the reactivity of a given complex.

\subsection{Relativistic effects in late transition metal catalysts}

The reason for gold complexes having high catalytic ability is due to the large relativistic effects in gold. What are relativistic effects? Relativistic effects are phenomena that are observed following the postulate that the speed of light, $c$ is invariant and independent of the frame of reference. Such phenomena include the increase in mass with increasing velocity as follows,

$$
m=\frac{m_{o}}{\sqrt{1-\left(\frac{v}{c}\right)^{2}}}
$$

where $m$ is the corrected mass, $m_{o}$ is the rest mass and $v$ is the velocity of the body.

Gold atoms, having an electron configuration of $[\mathrm{Xe}] 4 f^{14} 5 d^{10} 6 s^{1}$ (79 electrons), experience a large relativistic contraction of the core orbitals as the velocity of the electrons approaches $c$ around the heavy nucleus. The valence $6 s$ electron important for chemical reactions on the other hand, would be expected to move slowly $\left(v_{6 s} « c\right)$ even for gold and therefore has negligible relativistic effects. However, the opposite was observed instead as shown in Figure 1.6, with the contraction of $6 s$ orbital of gold to be the largest. While it is intuitive to explain this phenomenon from the contraction of inner orbitals that pulls in the valence orbitals, this is actually not the case.

The large relativistic contraction of the valence orbital is a direct action of the relativistic perturbation operator in the vicinity of the nucleus where the valence $s$ electrons are of significant part of its density (direct relativistic effect). Since the $s$ orbitals are stabilised 


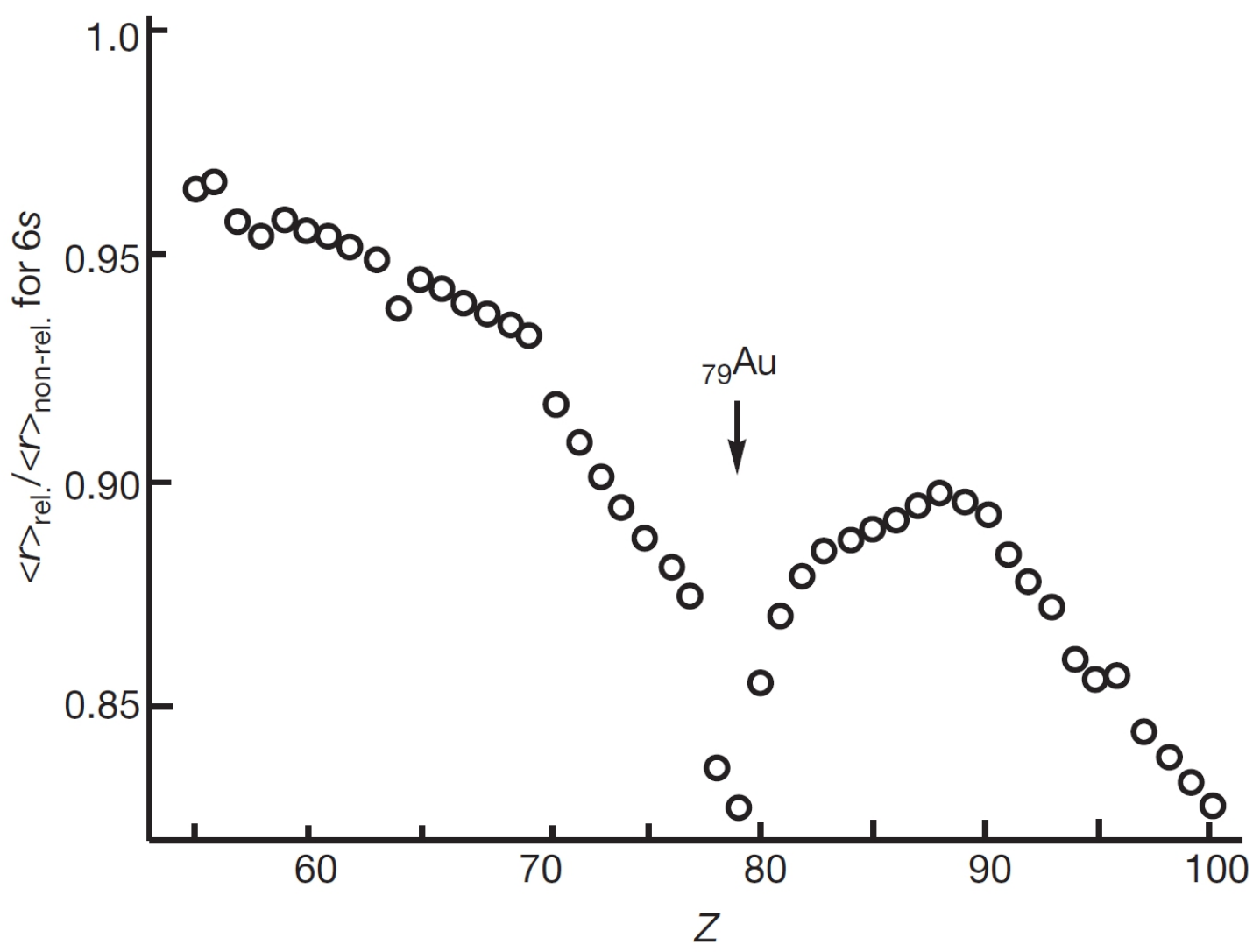

Figure 1.6 The ratio of relativistic $\left.(<\mathrm{r}\rangle_{\text {rel. }}\right)$ and non-relativistic $\left.(<\mathrm{r}\rangle_{\text {non-rel. }}\right) 6 s$-shell radii in atomic ground state. (Reproduced with permission; from Pyykkö and Desclaux ${ }^{104}$ copyright (c)1979 American Chemical Society.)

and closer to the nucleus, the shielding effect by the electrons in these orbitals increases, causing a lower effective nuclear charge experienced by electrons in the higher angular momentum orbitals. Hence, $d$ and $f$ orbitals expand radially and are energetically destabilised (indirect relativistic effect). The direct effects dominate in $s$ orbitals while the indirect effects dominate in $d$ and $f$ orbitals. As for $p$ orbitals, the direct and indirect effects almost cancel out each other. The relativistic contraction of the $s$ orbitals in gold atoms results in a greater ionisation energies for electrons in these orbitals. ${ }^{104-109}$ These effects are scalar relativistic effects which only show a decrease or increase in the orbital energies. Besides the scalar relativistic effects, spin-orbit coupling is another form of relativistic effect in atomic properties. It causes splitting in the energies of atomic orbitals, thus breaking the degeneracy of those orbitals (See Figure1.7) . 


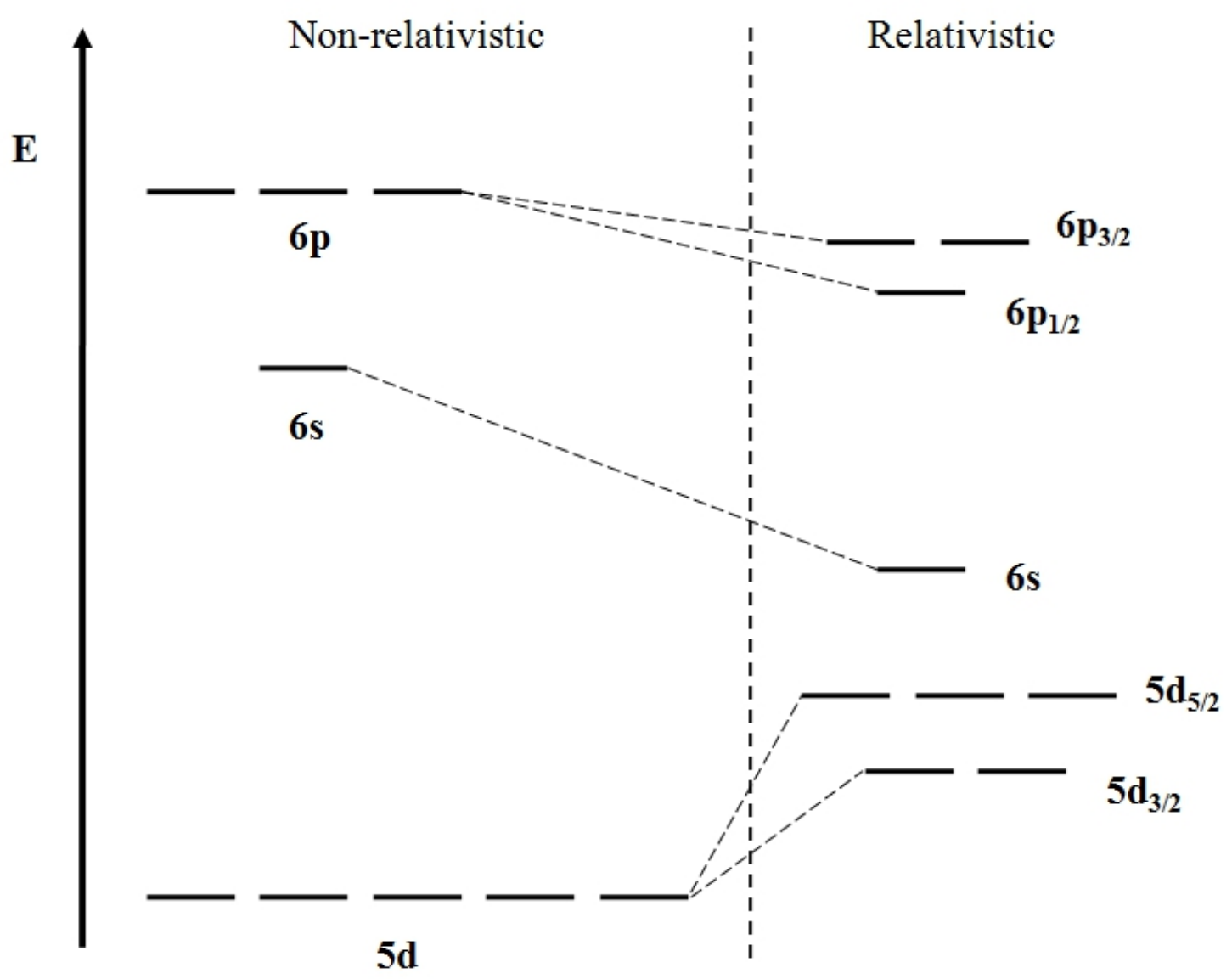

Figure 1.7 Relativistic and non-relativistic energy level diagrams of $5 d, 6 s$ and $6 p$ orbitals.

A review by Gorin and Toste summarises the relativistic effects in homogenous gold catalysis. ${ }^{66}$ Most of the reactivities of gold complexes as efficient catalysts are due to the relativistic effects they possess. As mentioned in the earlier sections, the high reactivity of $\mathrm{Au}$-alkyne activated complex towards nucleophilic attack is due to the relatively low lying LUMO of the complex which seems to be caused by the relativistic contraction of the valence $s$ and $p$ orbitals in gold atom. The insensitivity of gold catalysts towards air in most gold catalysed reactions can be explained by the electronic structure of gold. Compared to the $3 d$ electrons in $\mathrm{Cu}$, the $5 d$ electrons in $\mathrm{Au}$ experience less electronelectron repulsion due to the diffuse $5 d$ orbitals. Therefore, these electrons are held more strongly, resulting in a less nucleophilic metal centre that do not undergo oxidation reactions. ${ }^{110}$ 
The relativistic effects of gold also affect the bonding of its complexes. Calculations of $\mathrm{Au}(\mathrm{I})-$ ethyne and $\mathrm{Au}(\mathrm{I})-$ ethylene showed that backbonding contributes far less in the bonding energy than in the corresponding $\mathrm{Cu}$ complexes. ${ }^{111,112}$ The lack of backbonding from the metal to $\pi$-ligands e.g. alkyne is due to the antibonding orbitals of the alkyne being energetically too high for any significant backbonding to occur. This lack of backbonding causes the unsaturated hydrocarbon to be too electron deficient for nucleophilic addition to take place and explains the high Lewis acidity of gold complexes. However, experimental results on the formation of carbenoid species of gold complexes suggest a potential for backbonding to occur. ${ }^{113}$ Such backbonding was proposed to be attributed to the delocalisation of $5 d$ electrons into lower energy, empty, non-bonding orbitals. In other words, relativistic effects of gold directly affect the formation of intermediates in the mechanism and it is important to understand that these effects play a huge role in gold-catalysed reactions. Therefore, computational studies on gold catalysis have to include these relativistic effects in their calculations.

Pyykkö evaluated the vast difference in silver and gold chemistry even though they are in the same group of the periodic table. ${ }^{104}$ The results showed that the chemical difference between the fifth row and sixth row elements seemed to be mainly due to the large relativistic contribution which affects the chemical properties such as the shorter and stronger covalent bond formation by gold, the ionization potential and the electronic structure. The yellow colour of gold metal is due to the 'relativistic contraction' and 'relativistic expansion' of $s-p$ orbitals and $d-f$ orbitals, respectively. The decrease in band gap causes an electronic transition in the visible spectrum, where blue light is absorbed. Pyykkö concluded his report by stating that the relativistic effects were particularly strong around gold which was termed as the gold maximum. ${ }^{114}$ 


\subsection{Mechanistic investigation}

Every chemical reaction undergoes a step by step sequence of elementary reactions by which an overall chemical change occurs. This sequence of changes is what we call the reaction mechanism. A reaction mechanism provides a detailed description of how the reactants interact with each other to form the product(s). Several methods have been used to identify the intermediate species of a reaction such as labelling experiments, in situ spectroscopic techniques and trapping of intermediates. However, they do not provide detailed information of the kinetics and thermodynamics of a reaction such as the relative energy of the species involved and the energy barrier needed for a specific transformation in the reaction pathway. With the use of a computational approach, researchers are able to seek out new information in the reaction mechanism and provide more insights regarding the reaction. Computational methods have become one of the main tools in finding reaction mechanisms as increasing computer power and development of more sophisticated softwares allow for more accurate calculations of bigger and more complicated systems.

From the previous research, we believe that Teles and coworkers were the first to use a theoretical approach to investigate the mechanism of gold-catalysed nucleophilic additions. Teles et al. used ab initio calculation to predict the mechanism of the addition reaction of methanol to propyne as shown in Scheme 1.7. ${ }^{28}$ The results showed that the methanol attack occurred via an associative mechanism which involved the coordination to gold, forming an intermediate complex as shown. The structure of the intermediate clearly showed a syn addition of methanol to propyne. The transition state in the reaction pathway was sterically crowded and this explains the slower reactions of secondary alcohols compared to primary alcohols. From the $a b$ initio calculations, they established that the gold catalyst did not only activate the $\mathrm{C}-\mathrm{C}$ multiple bond but also acted as a proton shuttle to guide the hydrogen migration from the oxygen of methoxy group to 


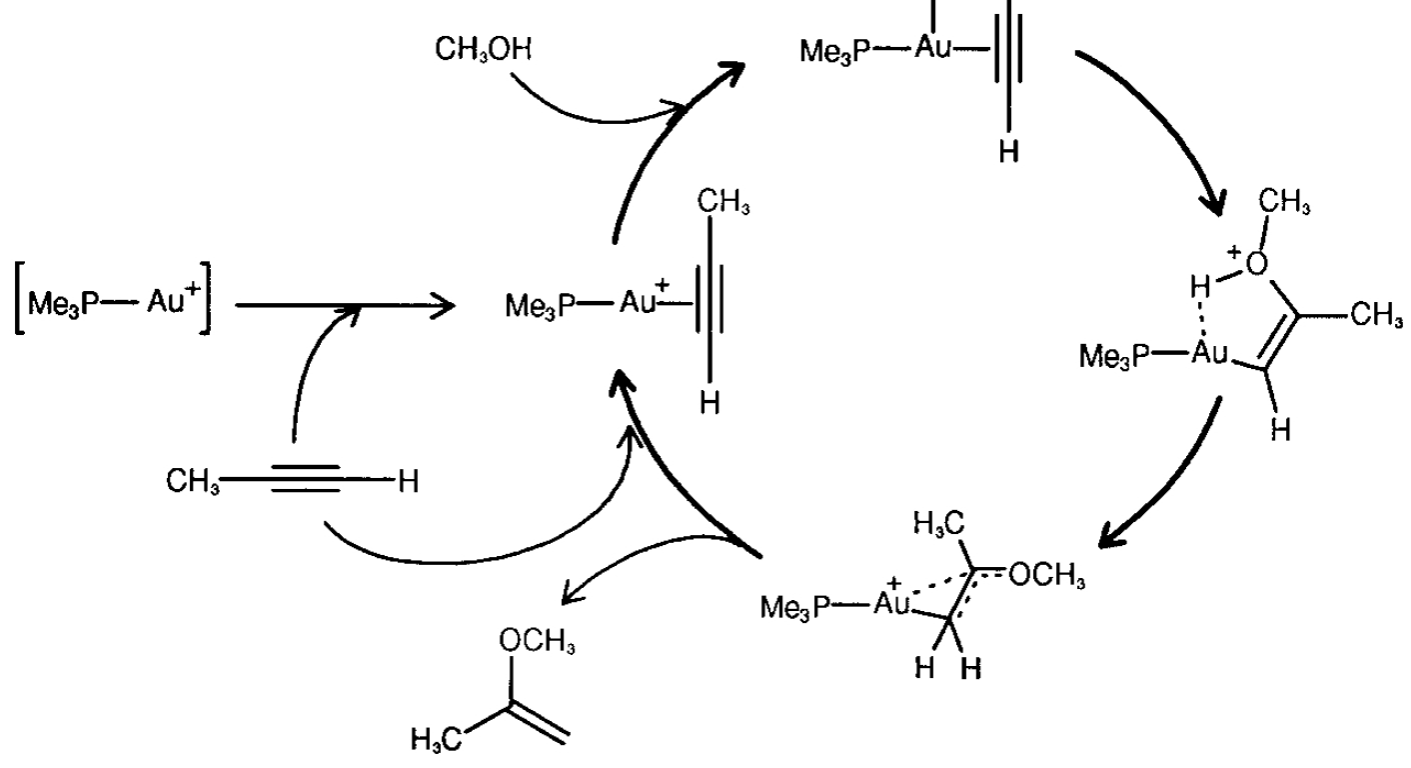

Scheme 1.7 Reaction mechanism for the addition of methanol to propyne proposed by Teles and coworkers. (Reproduced with permission; from Teles et al. ${ }^{28}$ copyright (C) 1998 Wiley.)

the carbon atom. Teles predicted that there might be another possible pathway which involves the deprotonation at the oxygen and reprotonation at the carbon atom but they failed to locate the transition state for this step. Their investigation was able to explain the stereoselectivity and the role of the catalyst in the mechanism but it failed to account for solvent effects that may arise. A number of theoretical studies have been reported since then and the summary of the progress in this field is depicted in Figure 1.8, showing the key research which has contributed greatly in this field and how the research relates to one and another.

The mechanism proposed by Teles et al. showed that methanol coordinates to the metal centre before it starts to react with the alkyne substrate. However, later theoretical study proved that the coordination of methanol to the metal centre did not occur in liquid phase and was merely just an artifact of the gas phase. ${ }^{115}$ The structure of the intermediates formed along a reaction pathway may seem trivial to discuss but it is one of the factors that determines the flow a reaction mechanism. There exist many different hypotheses for 


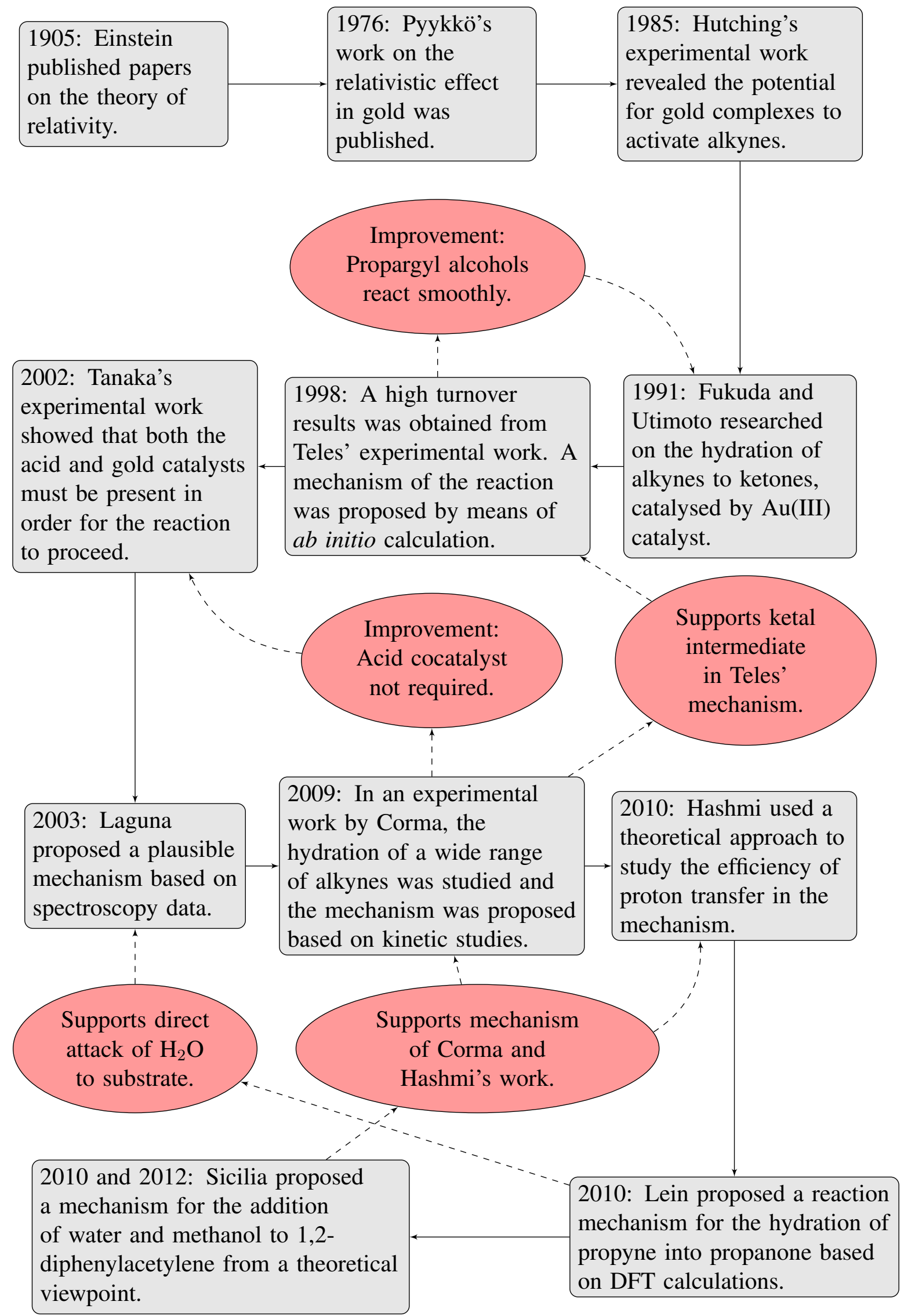

Figure 1.8 The connections between past research and their contribution to this study. 
the mechanism of hydration of alkynes due to the difficulty in identifying the structure of the intermediates. On one hand, Teles and coworkers mentioned enol ethers and ketals are plausible intermediates in the hydration of alkynes. On the other hand, Tanaka and coworkers proposed a direct attack of a awater molecule to the substrate in the transformation of alkynes to ketones based on their experimental results. ${ }^{23}$

To shed light on all of these, experimental work was done by Leyva and Corma to study the hydration reaction, with methanol as solvent and $\mathrm{Au}(\mathrm{I})$ cationic complexes as catalysts. ${ }^{56}$ Scheme 1.8 shows the mechanism proposed by them. They proposed that the ketone product was formed in the presence of water through the formation of enol ether and ketal as intermediates. The methanol molecule (instead of the water molecule) first attacks the activated alkyne to form an enol ether which then proceed to the formation of a ketal by another addition of methanol. Two isomers could be formed for enol ether, namely the $\mathrm{E}$ and $\mathrm{Z}$ isomers. The ratio of $\mathrm{Z}: \mathrm{E}$ increased with time when methanol was used as the only reagent while the less thermodynamically stable E isomer was found to be predominant over the $\mathrm{Z}$ isomer when water was used as the only reagent. Lastly, in the presence of water, the equilibrium shifts to the formation of ketone with the elimination of two methanol molecules from the ketal intermediate. The authors of this study proposed that the addition of the first methanol is more likely to occur than the addition of a water molecule. This is because the initial rate of the addition of methanol is higher than that of the addition of water.

A theoretical study by Sicilia and coworkers to investigate the mechanistic details of the hydroalkoxylation of 1,2-diphenylacetylene using methanol as nucleophile and $\left[\left(\mathrm{Ph}_{3} \mathrm{P}\right) \mathrm{Au}\right]^{+}$as the catalyst was recently conducted and the mechanism is depicted in Figure 1.9. ${ }^{116}$ The objective of this study was also to test the hypothesis from Leyva and Corma's experimental work using a computational approach. They found that the activation barrier for the first addition of methanol was lower than that of the addition of water molecule, supporting the first hypothesis by Leyva and Corma. A syn addition of 


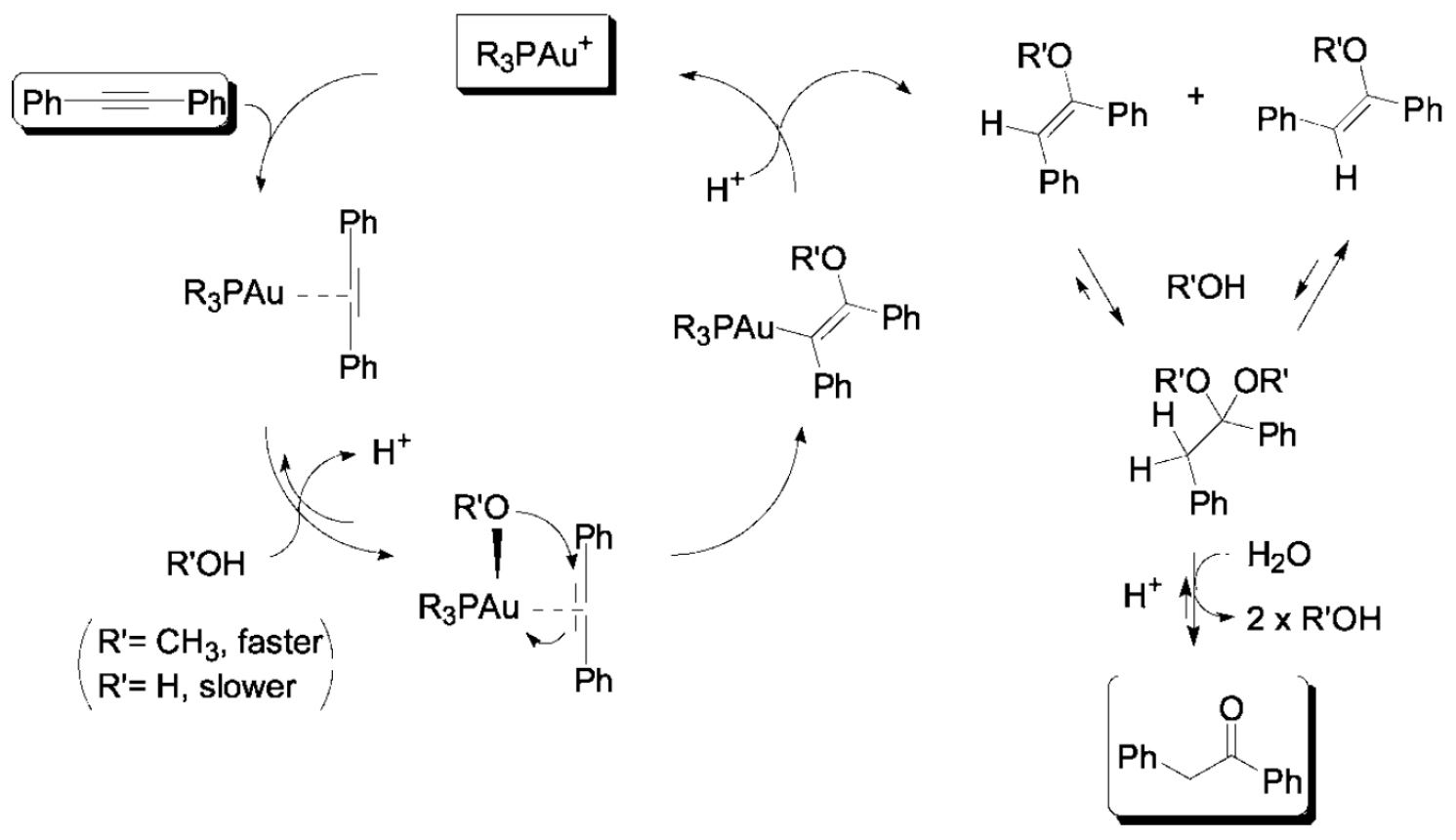

Scheme 1.8 Plausible mechanism for the hydration of alkynes using $\mathrm{AuPR}_{3}{ }^{+}$catalyst. (Reproduced with permission; from Leyva-Pérez and Corma ${ }^{56}$ copyright (C)2009 American Chemical Society.)

nucleophile to the $\mathrm{Au}(\mathrm{I})$-alkyne complex was found to be more favourable than the anti addition, resulting in the preferred formation of the $\mathrm{E}$ isomer of enol ether. However, the activation energy for the conversion of the $\mathrm{E}$ to the more stable $\mathrm{Z}$ isomer was very low and feasible. This again supports the explanation found in Leyva and Corma's study.

The second nucleophile attack posed some problems. Leyva and Corma reported that the preference towards the addition of methanol or water to enol ether cannot be concluded as they expect the protodeauration step to occur faster. Therefore, Sicilia and coworkers investigated two separate reaction pathways using methanol and water as nucleophiles. The addition of water to enol ether intermediates was preferred over the addition of methanol.

A point worthy of note is that ketone cannot be formed once the second methanol addition takes place. They tried to include the intervention of a water molecule to find a plausible transition state that corresponds to the release of methanol molecules from the ketal 


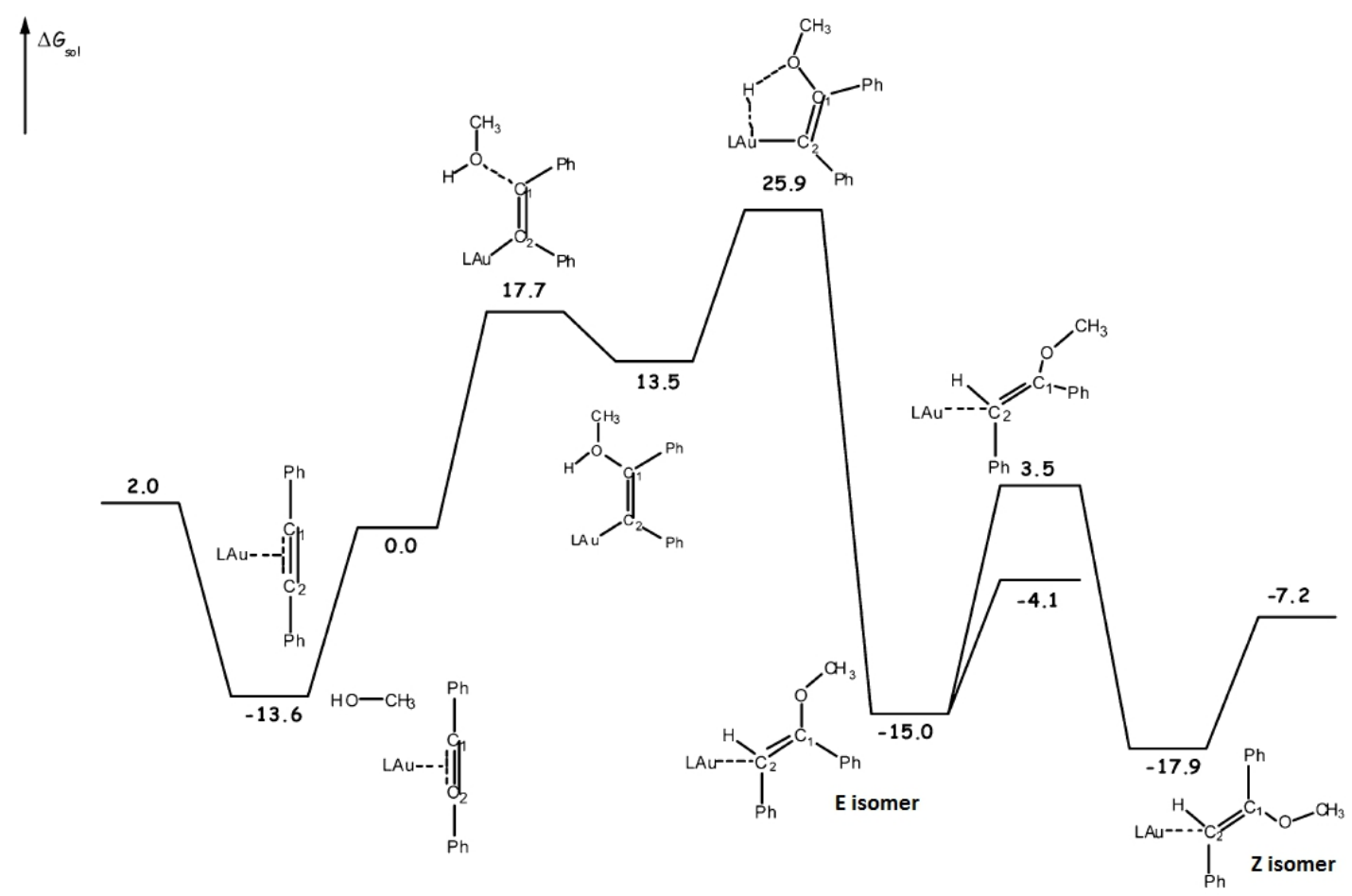

Figure 1.9 The mechanism for the first addition of methanol to 1,2-diphenylacetylene using $\left[\left(\mathrm{Ph}_{3} \mathrm{P}\right)-\mathrm{Au}\right]^{+}$complex as catalyst. Energies are in $\mathrm{kcal} \mathrm{mol}^{-1}$. (Reproduced with permission; from Mazzone et al. ${ }^{116}$ copyright (C)2012 American Chemical Society.)

intermediate but with no success in the end. On a positive side, the Au-hemiketal intermediate, formed from the addition of water to enol ether intermediate showed a clear pathway leading to the formation of ketone by releasing a methanol molecule as shown in Figure 1.10. It occurred via hydrogen migration from the hydroxyl group to the oxygen of methoxy group. Their findings were consistent with the experimental observation of Leyva and Corma, showing that the hydration of alkynes to ketones occurred via the formation of an enol ether and hemiketal as intermediates. In this case, the direct water addition to alkyne was considered somehow hampered based on the above mechanism together with the successful adaption of Teles' hydroalkoxylation method to the hydration reaction. Besides, the fact that alcohols are generally the best solvent ${ }^{27}$ for this kind of reaction while other solvents such as THF, DMF or toluene result in low yields suggests that the addition of alcohol is the key step as opposed to the direct addition of water. 


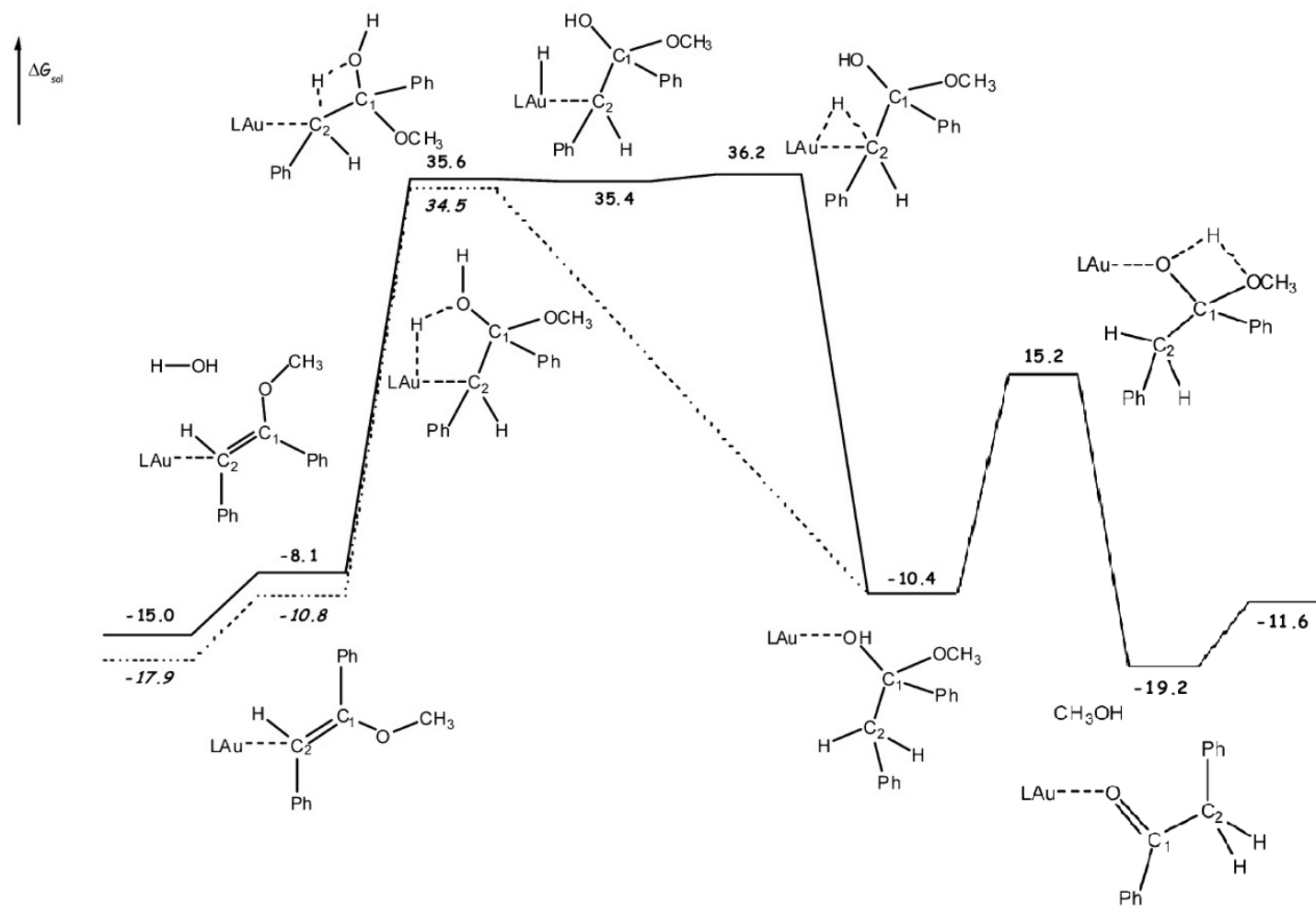

Figure 1.10 The mechanism for the second nucleophilic addition of water to both enol ether $\mathrm{E}$ (solid line) and enol ether $\mathrm{Z}$ (dashed line) isomers using $\left[\left(\mathrm{Ph}_{3} \mathrm{P}\right)-\mathrm{Au}\right]^{+}$complex as catalyst. Energies are in $\mathrm{kcal} \mathrm{mol}^{-1}$. (Reproduced with permission; from Mazzone et al. ${ }^{116}$ copyright (C)2012 American Chemical Society.)

However, the basis of this claim is not enough to eliminate the possibility of direct water addition to alkyne completely. In the year 2003, Laguna and coworkers proposed a mechanism on the direct addition of water molecule to activated alkyne when catalysed by $\mathrm{Au}(\mathrm{III})$ complexes. ${ }^{25}$ Their mechanism was supported by spectroscopy data at different temperature and it is depicted in Scheme 1.9. A theoretical investigation by Lein and coworkers showed that the direct attack of $\mathrm{H}_{2} \mathrm{O}$ was energetically feasible and further strengthens the claim for this step.

In 2010, Lein et al. studied the mechanism of hydration of propyne using density functional theory and $\mathrm{AuCl}_{3}$ as the catalytic complex. ${ }^{117}$ In contrast with Teles' results, Lein and coworkers found that the attack of water molecule on propyne proceeds via an anti addition and the transition state was stabilised by the hydrogen bonding network 


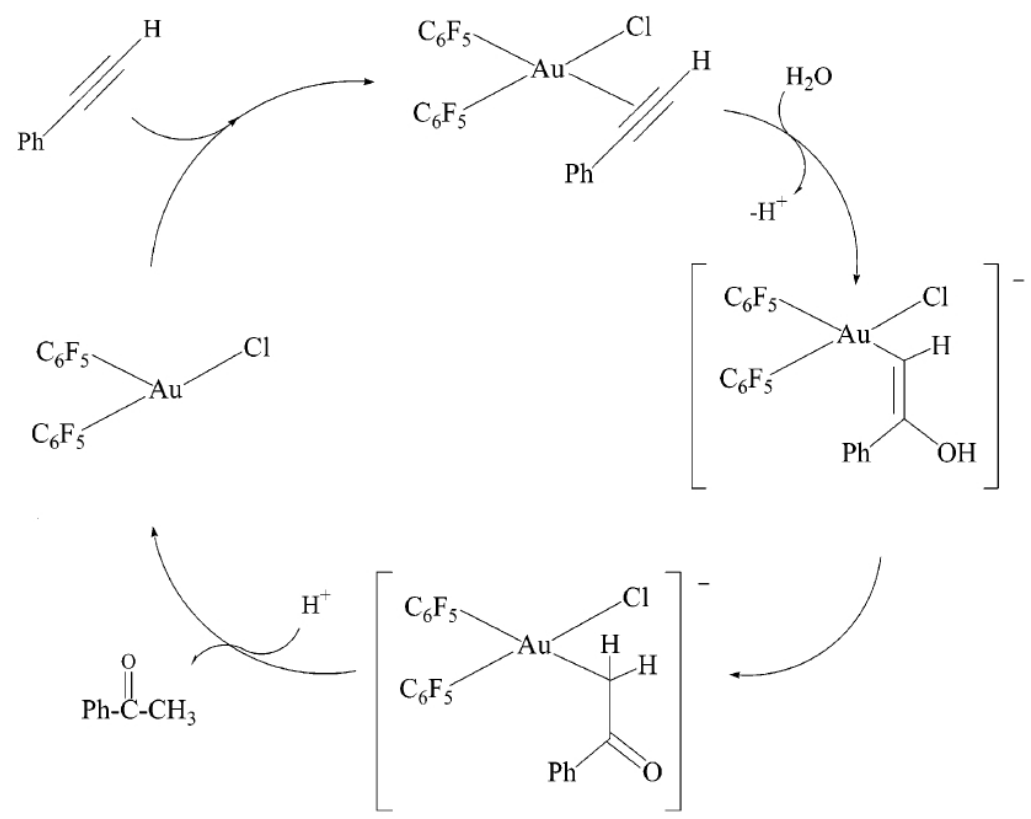

Scheme 1.9 Schematic reaction mechanism proposed by Laguna and coworkers. (Reproduced with permission; from Casado et al. ${ }^{25}$ copyright (C)2003 American Chemical Society.)

with gold moiety in the complex structure. The mechanism showed that a second water molecule is needed to lower the activation barrier in the reaction pathway. It also acted as a mediator to guide the migration of hydrogen near the end of the pathway. This finding is in agreement with the results found by Hashmi and coworkers. ${ }^{115}$ They reported that the water molecules did not only assist the hydrogen migration and facilitate the addition step but also to hold the water nucleophile in the right position for an anti addition. The reaction mechanism presented in the article explained how the participation of solvent molecules can lead to an overall more efficient pathway that would justify the high turnover frequency of the reaction. The details of this paper will be discussed later in this section.

By using a nonrelativistic basis set and another one that includes a relativistic pseudopotential core for the gold atom, Lein and coworkers managed to evaluate the relativistic effects on their molecular system. The nonrelativistic pathway appeared to be much 
shallower than the relativistic one, indicating a weaker interaction of the nonrelativistic gold atom with the substrate molecule but the nature of the path and energy barrier appeared to be similar. It was also reported that the reduction of $\mathrm{Au}(\mathrm{III})$ complex to $\mathrm{Au}(\mathrm{I})$ complex was favourable when the calculation was done nonrelativistically whereas relativistic calculations favoured the $\mathrm{Au}(\mathrm{III})$ complex. In other words, the first step of the catalytic cycle proposed was not even feasible in a hypothetical nonrelativistic condition due to the decomposition of the $\mathrm{Au}(\mathrm{III})$ complex into $\mathrm{Au}(\mathrm{I})$ and $\mathrm{Cl}_{2}$. This proved that the catalytic ability of $\mathrm{Au}(\mathrm{III})$ was indeed a relativistic effect. The overall mechanism is depicted in Figure 1.11. 


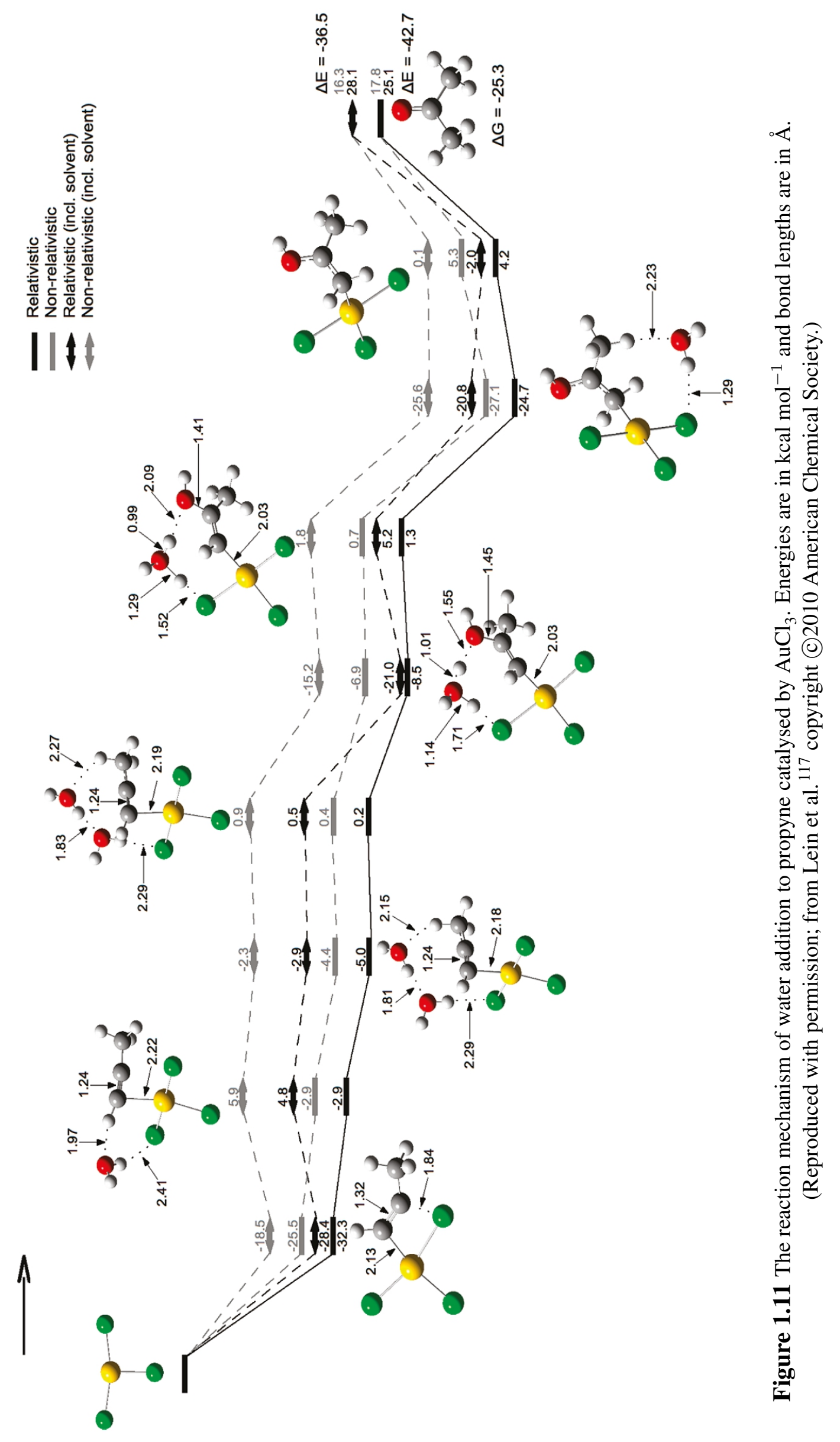


The participation of solvent molecules was important for an efficient reaction as reported in Lein's study. There are many different possibilities in which the solvent molecules can be arranged alongside the complex and the participation of solvent molecules leads to a more efficient reaction pathway with lower activation energy. In the same year Lein's study was published, Hashmi and coworkers reported the solvent effects on proton transfer in the mechanism of $\mathrm{Au}(\mathrm{I})$-catalysed hydration of alkynes from a theoretical viewpoint. ${ }^{115,118}$ Figure 1.12 shows the energy profile of the reaction and the activation energy was found to be $12.3 \mathrm{kcal} \mathrm{mol}^{-1}$ for the addition of water to propyne at the central carbon and $16.8 \mathrm{kcal} \mathrm{mol}^{-1}$ for the proton transfer from the coordinated water molecule to the terminal carbon. A similar mechanism but with arrangement of water molecules around the complex to enable a more efficient proton transfer step was then found and the energy barrier corresponding to the proton transfer was only $3.7 \mathrm{kcal} \mathrm{mol}^{-1}$. This clearly shows that the participation of solvent molecules in the mechanism significantly lowers the barrier for the proton transfer step.

Knowing that the participation of solvent molecules lowers the barrier for the proton transfer step, Hashmi and coworkers further investigated the effects of solvent on the water addition step as well. A properly arranged solvent cage surrounding the complex was modelled and using the same method as before, a new mechanism was found for the solvent-assisted pathway. Remarkably, the activation energy for the addition step was also significantly lowered to $4.2 \mathrm{kcal} \mathrm{mol}^{-1}$ (Figure 1.13) as opposed to $12.3 \mathrm{kcal} \mathrm{mol}^{-1}$ in the "unassisted" mechanism. It is worthy of note that an increase of the number of solvent molecules in the cage had caused a slight decrease in the activation energy of the proton transfer step. However, the correlation between the number of solvent molecules and the activation energy was not reported in the paper.

They proceeded to investigate the mechanism of nucleophilic addition of methanol to propyne using the same solvent (water) cage. As expected, the role of the solvent cage as a proton acceptor in the addition and proton transfer step resulted in a lower energy 


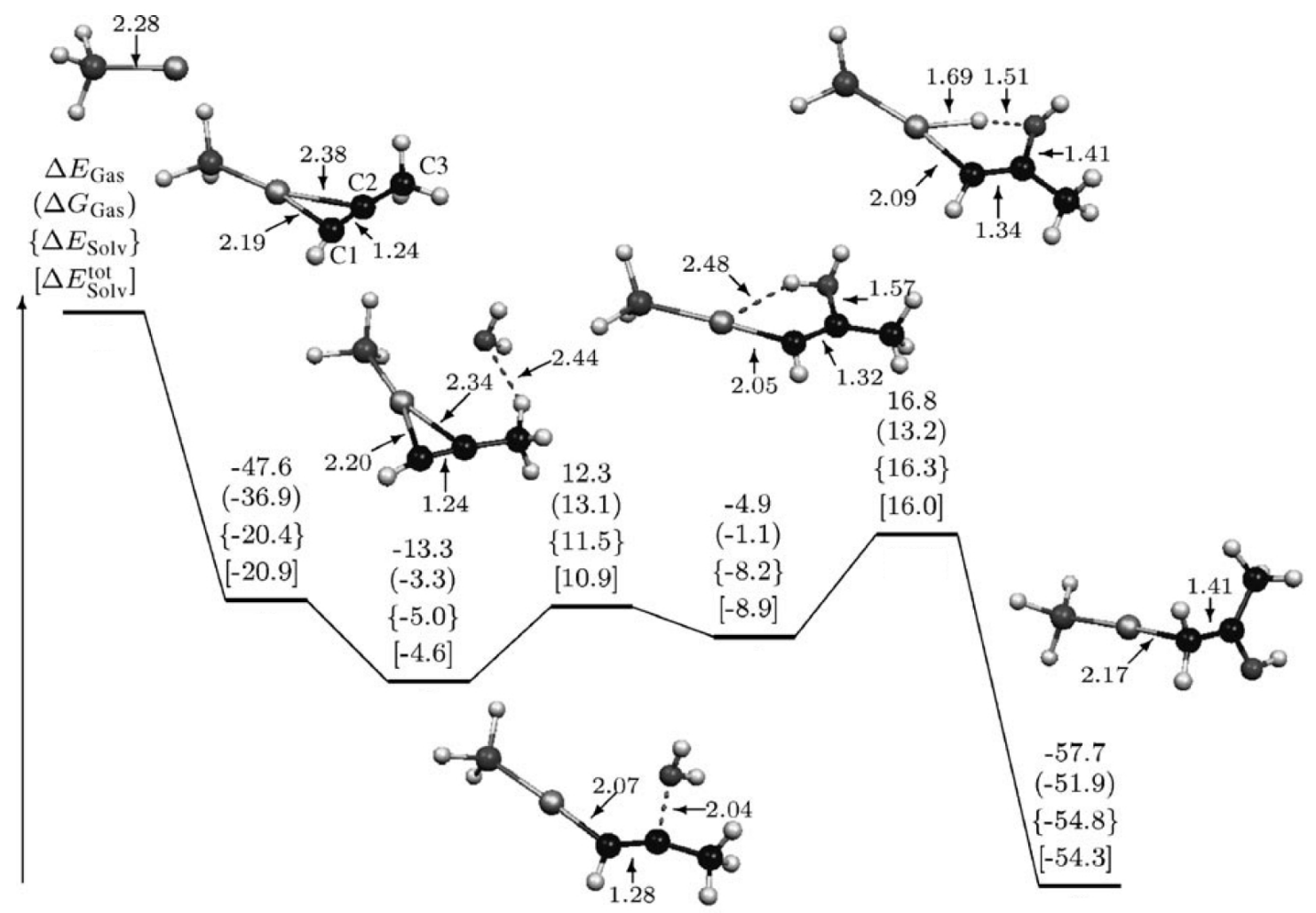

Figure 1.12 Energy profile of $\mathrm{Au}(\mathrm{I})$ catalysed addition of water to propyne (without assistance by water). Energies are in $\mathrm{kcal} \mathrm{mol}^{-1}$ and bond lengths are in $\AA$. (Reproduced with permission; from Krauter et al. ${ }^{115}$ copyright (C) 2010 Wiley.)

barrier. Comparing the addition of methanol to the addition of water to propyne, the calculated activation energies in both these reactions were almost identical, with a slight preference over the addition of methanol. Further investigation on the proton transfer step in hydration of alkynes lead them to an interesting study on the triflate-assisted mechanism instead of the conventional water-assisted proton transfer. The overall proton transfer of this type was found to be slightly higher in energy than the ones in addition of water and methanol but it was still feasible under the reaction conditions. The effects of solvent cage, e.g. the number of solvent molecules and the shape of the cage, on the activation energy of the addition and hydrogen migration step has not been explored to the best of our knowledge.

The position in which the nucleophile attacks is an important aspect in a reaction 


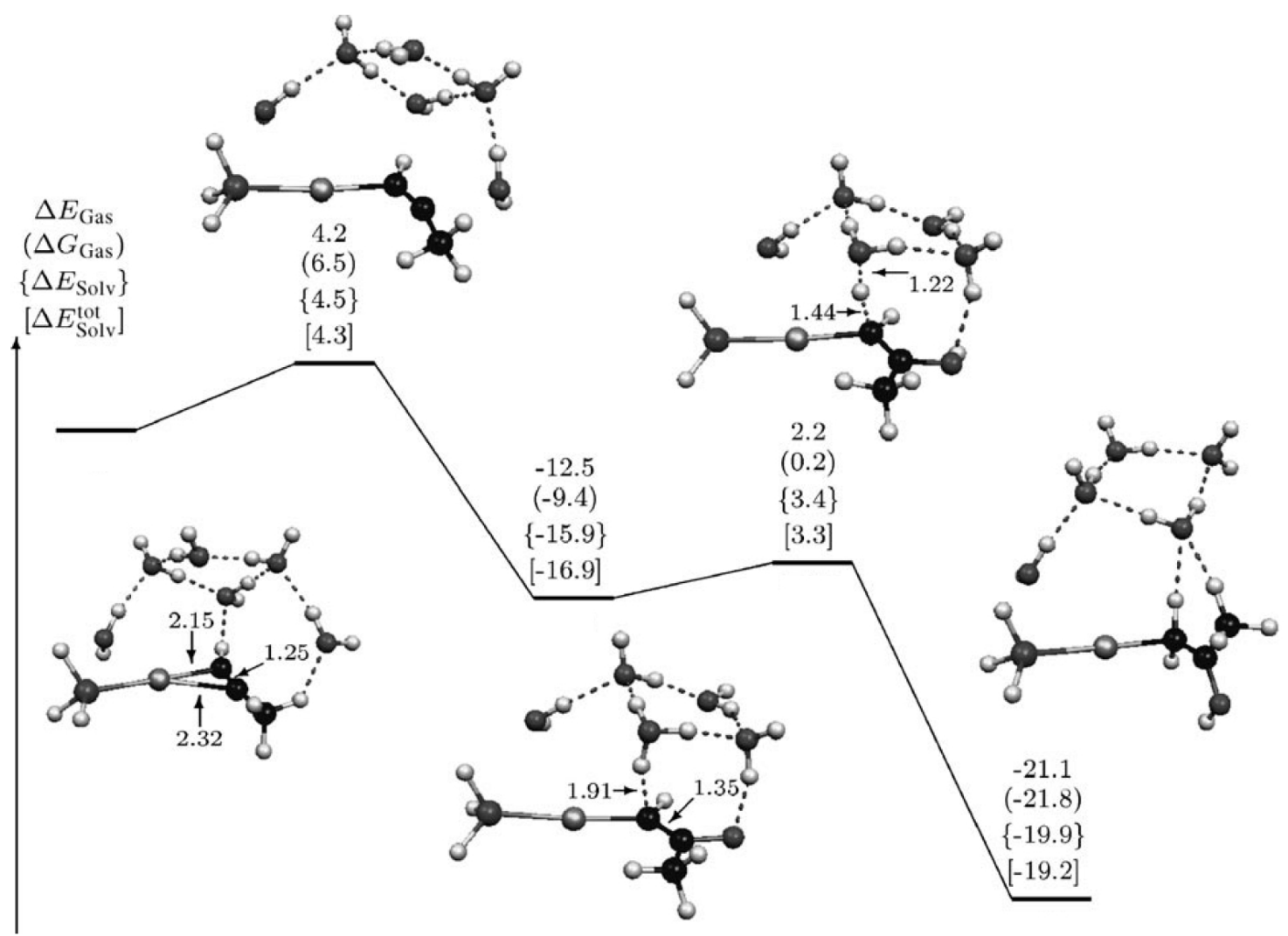

Figure 1.13 Energy profile of $\mathrm{Au}(\mathrm{I})$ catalysed addition of water to propyne (assisted by water). Energies are in $\mathrm{kcal} \mathrm{mol}^{-1}$ and bond lengths are in $\AA$. (Reproduced with permission; from Krauter et al. ${ }^{115}$ copyright (C) 2010 Wiley.)

mechanism. Teles proposed a mechanism showing that the nucleophile attack occurred on the same side (syn position) as the catalyst in the alkyne-gold complex. However, in later studies, some showed that the anti-addition ${ }^{25,44,117,119,120}$ was preferred over the $s y n$-addition ${ }^{28,30,116,121}$ while others supported the original proposal by Teles. ${ }^{28}$ The position in which the nucleophile attacks was affected by the ligands attached to the catalyst. As we can see from Lein's investigation, the preference towards anti-addition might be because that the syn position is sterically hindered by one of the chloro ligands. Consistent with this, Sicilia and coworkers have calculated that the syn-addition was slightly more energetically preferred than the anti-addition for the addition of water and methanol to 1,2-diphenylacetylene catalysed by $\mathrm{Au}(\mathrm{I})$ complex. The $\mathrm{Au}(\mathrm{I})$ catalyst used in the study had only one ligand and did not hinder the syn-addition. In fact, such 
orientation allowed gold to directly participate in assisting the proton transfer leading to the formation of product.

Although gold compounds were known to have catalytic properties in the addition of alcohols to alkynes more than a decade ago, little is known about the mechanism of such catalysed reactions. The study on the mechanism of this reaction is important to get a better understanding on how the catalyst activates the reactants and further improvement can be made on the catalyst by taking advantage of the information provided by the mechanism. It also gives a better insight on the regioselectivity along the reaction pathway. Gaining that kind of knowledge is important in synthetic chemistry as it provides a platform for chemists to alter the reaction conditions or the structure of their starting compounds in order to obtain the desired product. Last but not the least, with the knowledge of the reaction mechanism, different gold-catalysed reactions can be compared using the information of the calculated activation energy of the respective steps. 


\section{Chapter 2}

\section{Methodology}

\subsection{Computational chemistry methods}

Computational chemistry combines the use of mathematical methods with the laws of physics to study the properties of chemical systems. The behaviour and interaction between matter at the atomic or subatomic scale such as electrons cannot be described accurately using the laws of classical physics. This is where quantum mechanics comes into play and the equation that allows for the calculation of a system's energy is the timeindependent Schrödinger equation (2.1)

$$
\mathbf{H} \psi=E \psi
$$

where, $\mathbf{H}$ is the Hamilton operator, $\psi$ is an eigenfunction that describes the nature of the chemical systems and $E$ is the energy of the system. However, the equation can be solved exactly only for systems with one or two particles. When chemical systems get more complicated, the solution becomes impossible to calculate analytically. Many methods are used to solve more complex systems, ranging from the lower level Hartree-Fock (HF) method to very expensive and accurate methods such as full configuration interaction (FCI).

The complexity of a many-body system can be simplified computationally by treating the particles independently as proposed by HF theory. This means that the electrons in a molecule move and behave with respect to the mean field generated by the other electrons. The interaction between the electrons in the system is approximated by getting an average of all the interactions in the system. Since HF theory only accounts for the 
average interaction between the electrons, the HF model has only limited accuracy. The interaction between electrons that is considered in HF model follows the Pauli principle which prevents an electron pair in the same spatial MO to be of the same spin. This is known as Fermi correlation. Even without considering the full correlation between electrons, the HF approach often provides approximately $99 \%$ of the correct energy. The missing $1 \%$ is the Coulomb correlation energy (or normally just referred to as electroncorrelation) which holds the key to describe the system accurately. The development of computational methods such as configuration interaction (CI), the Møller-Plesset perturbation theory (MP) and coupled cluster (CC) method focuses on recovering the electron-correlation to get closer to the exact energy. However, the computational effort increases drastically with the size of the basis set used and the number of determinants. Only the electron-correlation of very small systems can be recovered fully using these methods, such as full-CI, without too much computational effort. This is not practical for computational studies that investigate the properties of large molecules.

In order to improve results beyond HF without high computational cost, most computational studies utilise the Density Functional Theory (DFT) method which is based on the Hohenberg-Kohn theorems. The theorems state that the ground state electronic energy can be determined by the electron density, $\rho$ of the system. The superiority of DFT methods in terms of computational effort can be seen by comparing them with the wave function approach. In wave function methods, a system of $N$ electrons contains $4 N$ variables, three spatial and one spin coordinate for each electron. On the other hand, in the DFT approach, one computes the electron density which is the square of the wave function, integrated over $N-1$ electron coordinates and the number of variables is reduced from $4 N$ to only four coordinates ( 3 spatial and 1 spin coordinates) regardless of the number of electrons. However, the flaw of this orbital-free model is that the kinetic energy is poorly represented, resulting in the accuracy to be too low for general use.

As a solution to that, Kohn and Sham introduced a concept which has now become 
the foundation for the use of DFT in computational chemisty. In order to get a good representation of the system's energy, the kinetic energy functional is divided into two parts which consist of one that can be calculated exactly and a small correction term. The consequence of this improvement is the re-introduction of orbitals that increases the variables from 4 coordinates to $4 N$ coordinates; $3 N$ spatial and $N$ spin coordinates. The Kohn-Sham theory calculates the kinetic energy by assuming that the electrons are non-interacting in the same way as the HF model does and the solution to this can be easily given in the form of a Slater determinant. This approach recovers most of the system's energy which consists of the kinetic energy, $T_{\mathrm{S}}$, attraction between electrons and nuclei, $E_{\mathrm{ne}}$ and the classical electron-electron repulsion, $J$ as can be seen in Equation 2.2.

$$
E_{\mathrm{DFT}}[\rho]=T_{\mathrm{S}}[\rho]+E_{\mathrm{ne}}[\rho]+J[\rho]+E_{\mathrm{xc}}[\rho]
$$

But in reality, the electrons are in fact interacting. The difference between the exact energy and the calculated energy under the assumption of non-interacting orbitals is very small. This is similar to how the HF model is able to provide approximately $99 \%$ of the correct energy. The difference is included in a correction term known as exchange-correlation term, $E_{\mathrm{xc}}$ as can be seen in Equation 2.3,

$$
E_{\mathrm{xc}}[\rho]=\left(T[\rho]-T_{\mathrm{S}}[\rho]\right)+\left(E_{\mathrm{ee}}[\rho]-J[\rho]\right)
$$

where $T$ is the kinetic energy of an interacting system and $E_{\mathrm{ee}}$ is the interaction between electrons in an interacting system.

Kohn-Sham theory focuses only on deriving the approximations to the exchangecorrelation energy functionals while the orbital-free model's task is to derive approximations to all three components, i.e. the kinetic, exchange and correlation energy functionals. The main problem of the DFT approach in general is that there is no systematic way of improving the results. There exist many DFT methods, e.g. local density approximation (LDA), generalised gradient approximation (GGA), meta-GGA and hybrid, due to a high 
number of functionals used to approximate the exchange-correlation energy. The general hierarchy of chemical accuracy produced by DFT methods is as shown in the Jacob's Ladder of chemical accuracy (Figure 2.1). The local density approximation (LDA) assumes that the density can be treated as a uniform electron gas or that the density is a slowly varying function. LDA has been used extensively in describing extended systems such as metals where the approximation of slowly varying electron density is valid. An improvement on LDA is to consider electron density as a non-uniform electron gas. This is done by making the exchange and correlation energies depend on the electron density and the first derivative of the density as well which is now known as GGA. Meta-GGA is similar to GGA but with the exchange and correlation functionals depend on higher order derivatives of the electron density. One of the ways to improve DFT results is to include a fraction of exact HF exchange into the exchange-correlation term and this results in a hybrid functional. Part of this improvement is that the exact HF exchange would reduce the self-interaction error (electrons interacting with itself) within DFT. A functional can also be made double hybrid by including not just a certain fraction of exact exchange but also a certain amount of perturbative second-order correlation.

Despite the many successes of DFT in quantum chemistry calculations, there are some areas where the current functionals perform very poorly. One of the main DFT problems is poor performance when describing weak interactions from dispersion forces, which arise from electron correlation in wave function methods. Most functionals underestimate the attraction forces in these weak interactions and some even predict a repulsive energy curve. The functionals in DFT do not have the correct $\mathrm{R}^{-6}$ limiting behaviour in the long range limit which causes their downfall in describing systems dominated by weak interactions. Some procedures, however, have been implemented to improve this aspect of DFT such as the inclusion of dispersion corrections in the functionals. As an example, the D2 and D3 dispersion corrections developed by Grimme ${ }^{122,123}$ have been used extensively in studies to improve the description of long range interactions. 


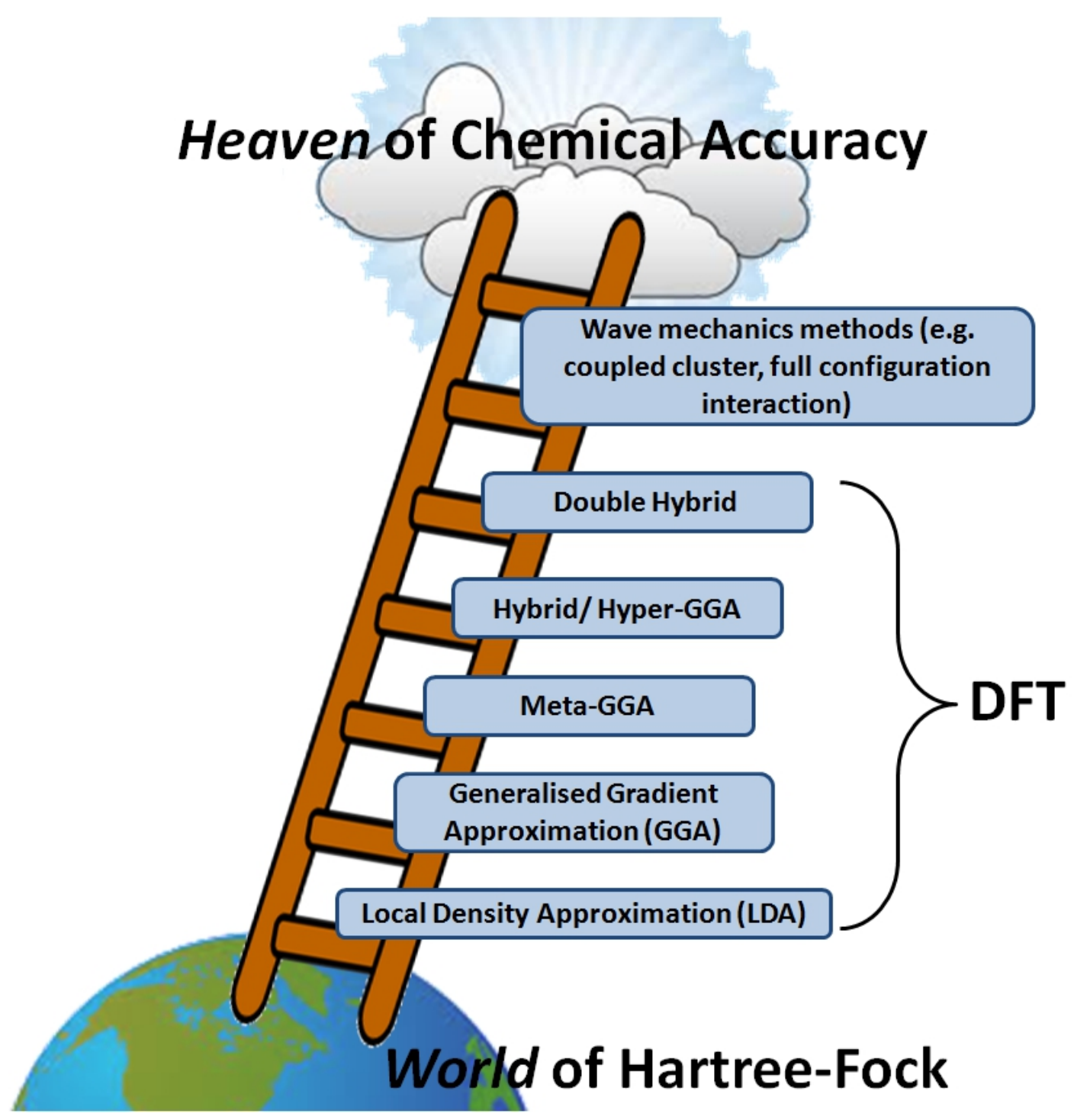

Figure 2.1 Jacob's ladder of chemical accuracy.

Choosing the correct functionals for the system under study is crucial to produce reliable results. A study was conducted by Kang et al. using different DFT methods and some other ab initio methods on different $\mathrm{Au}(\mathrm{I})$ and $\mathrm{Au}(\mathrm{III})$ complexes $\left(\mathrm{Au}^{+}, \mathrm{AuCl}\right.$, $\mathrm{AuCl}_{3}, \mathrm{Au}\left(\mathrm{C}_{3} \mathrm{H}_{4} \mathrm{~N}_{2}\right)^{+}$and $\left.\mathrm{AuPH}_{3}{ }^{+}\right)$with various unsaturated aliphatic hydrocarbons (ethene, ethyne and allene). ${ }^{124}$ The merits of the different methods were assessed based on high-level coupled cluster calculations with core-valence correlation $(\operatorname{CCSD}(\mathrm{T}))$ and complete basis set (CBS) limit extrapolation as reference. They made use of several molecular properties such as the structure (key bond lengths) and the bond dissociation 
energies calculated by different methods as parameters for their comparison. By obtaining all values from the methods, they were able to get the mean signed and unsigned deviations from $\operatorname{CCSD}(\mathrm{T}) / \mathrm{CBS}_{\mathrm{final}}$.

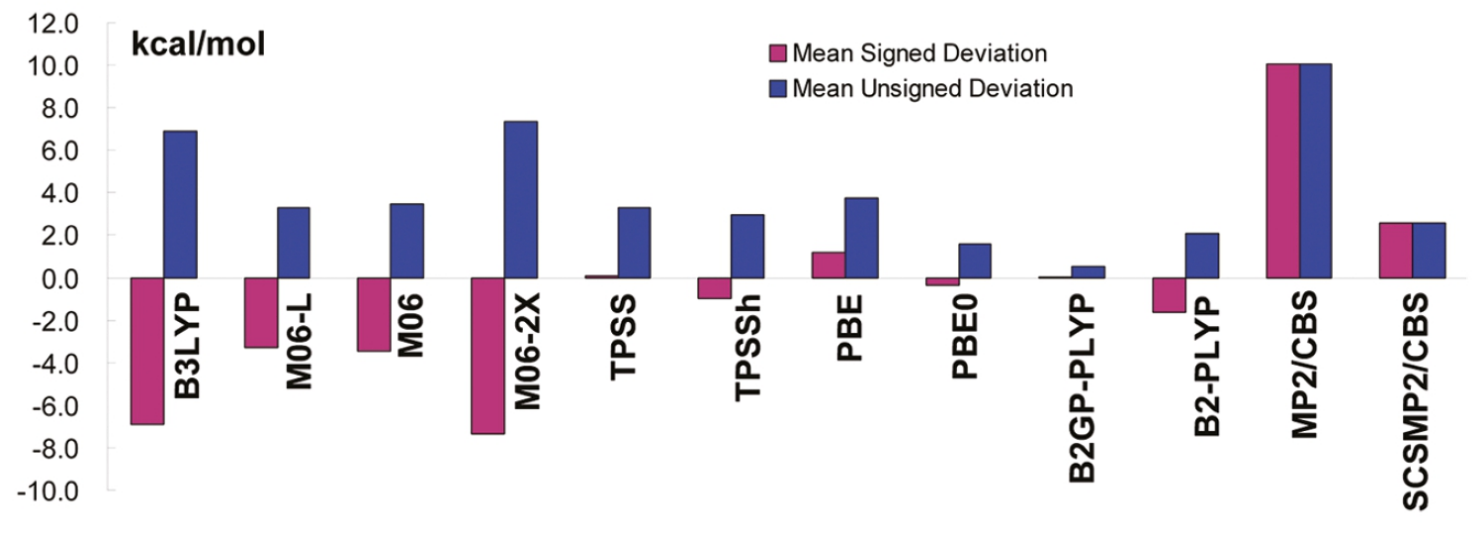

Figure 2.2 Mean signed and unsigned deviations of calculated bond dissociation energies with various DFT/ATZ and ab initio methods taking $\operatorname{CCSD}(\mathrm{T}) / \mathrm{CBS}_{\text {final }}$ as reference. ${ }^{124}$

Their findings showed that the three best performing functionals were the double-hybrid functionals B2GP-PLYP and B2-PLYP, and the single-hybrid functional, PBE0 for the complexes under study. Ab initio MP2 was the worst performing method as shown in Figure 2.2. They also evaluated the functionals when Grimme's D3 dispersion corrections but it was shown that these corrections do not uniformly affect the results. The correction made the B2-PLYP results better but worsen the B2GP-PLYP functional while it has little to no effect on the PBE0 functional. Among the three best functionals reported in Kang's paper, PBE0 was chosen as the functional used in this project due to the lower computational cost a single-hybrid functional requires compared to double-hybrid functionals. From Figure 2.2, the accuracy of the results does not seem to be compromised when PBE0 was used compared to the best B2GP-PLYP double-hybrid functional. Additionally, the BP86 functional is also used for the first part of this thesis so that the results can be compared with the study by Lein and coworkers on the $\mathrm{AuCl}_{3}$-catalysed reaction of propyne and water. The PBE0 functional consists of Perdew, Burke and Ernzerhof's ${ }^{125,126}$ pure functional which was made into a hybrid by Adamo. ${ }^{127} \mathrm{BP} 86$, on 
the other hand is a generalised gradient approximation (GGA) DFT that includes Becke's 1988 exchange functional ${ }^{128}$ combined with Perdew's local correlation functional. ${ }^{129}$ The difference between hybrid and GGA functionals is that hybrid functionals include a mixture of HF exchange energy into DFT exchange-correlation which can be calculated exactly using the HF wave mechanics method.

\subsection{Relativistic methods}

When one talks about the relativity in quantum chemistry, the term 'relativistic effects' is often used. This term is somewhat misleading because the effects as mentioned in Chapter 1.5 are in principle unphysical. The effects are not distinguishable as there is no distinction between a 'non-relativistic' and a 'relativistic' atom. These effects are basically the results of two different theoretical models, Schrödinger and Dirac model, that are used. If we consider the time-dependent Schrödinger equation (2.4), we see that the derivatives of the spatial coordinates are of second order but the time derivative is only first order. This is not consistent with the postulate of special relativity that physical laws are invariant in all coordinate system. Therefore, the fundamental structure of Schrödinger equation is not relativistically correct.

$$
\left[-\frac{1}{2 m}\left(\frac{\partial^{2}}{\partial x^{2}}+\frac{\partial^{2}}{\partial y^{2}}+\frac{\partial^{2}}{\partial z^{2}}\right)+\mathbf{V}\right] \psi=i \frac{\partial \psi}{\partial t}
$$

When the solutions $\psi$ in the many electron fields are used as the one-electron states in a single Slater determinant with the Hamiltonian, we get the relativistic Hartree-Fock which is also known as the Dirac-Fock equation.

$$
\left(c \alpha \cdot \mathbf{p}+\beta m c^{2}+e \mathbf{V}(\mathbf{r})\right) \psi(\mathbf{r})=E \psi(\mathbf{r})
$$


where $\psi$ is the one-electron wave function with rest mass, $m, E$ is the energy of the one-electron system, $\mathbf{p}$ is the momentum operator, $c$ is the speed of light, $e$ is the charge of the electron, $\mathbf{V}(\mathbf{r})$ is the potential energy, $\alpha$ and $\beta$ are the $4 \times 4$ Pauli matrices with their squares equal to identity matrix. The Dirac equation is four-dimensional and the relativistic wave function consequently contains four component vectors; two of which are assigned as intrinsic electron spins while the other two are interpreted as two different particles, electron and positron. The main difference between the Schrödinger and Dirac equation is the positronic solutions found in Dirac which show up as continuum of negative energy states (Figure 2.3).

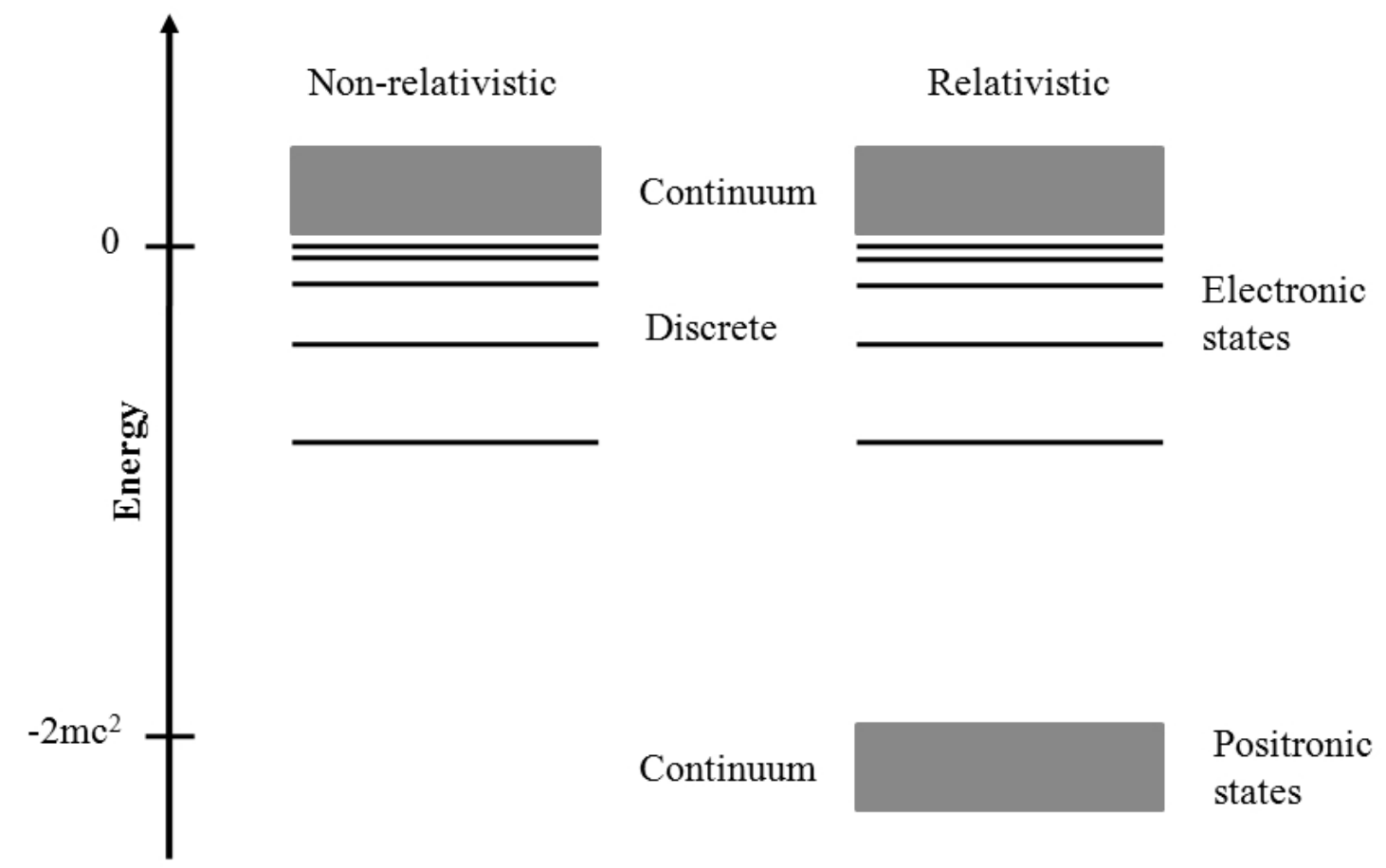

Figure 2.3 The difference between Schrödinger and Dirac solutions.

Explicit relativistic calculations are extremely high in computational cost because it requires the electrons to be described in four component vectors. One of the way to reduce the computational requirement is to reduce the number of components in the wave function to two. The Douglas-Kroll-Hess (DKH) theory ${ }^{130-133}$ manages to accomplish that by decoupling the positive and negative energy states of the Dirac Hamiltonian 
since only the positive energy states i.e. the electronic bound states are of interest in chemistry. The advantage of this approach is that the resulting two-components Hamiltonian produces the exact same energy eigenvalues as the four-components Dirac operator but with less computational requirement.

The most common way to include relativistic effects in calculations is by using relativistically parameterized effective core potentials which is the approach this work employs. The core potentials are created by fitting a potential function to the electron density distribution from a relativistic calculation for that particular atom. These core potentials have to be used along with the valence basis set that was created for the use of that particular core potential. This method is mostly used in literature because it includes relativistic effects on the core electrons and at the same time increases the speed of calculation by replacing a large number of electrons with a core potential.

\subsection{Basis sets}

As well as using the appropriate functionals in a computational study, basis sets also play an important role in describing the molecular system and ultimately producing reliable results. Basis sets consist of a linear combination of a set of basis functions which give a representation of the molecular orbitals of the system. Molecular orbitals can be described by a set of basis functions exactly if the basis set is complete. However, a complete basis set consists of an infinite number of functions and it is impossible to be solved by Schrödinger's equation in an actual calculation. When an incomplete i.e. finite basis set is used, the description of the components in a molecular orbital is limited to the type of basis functions and the direction in the spatial coordinates of which the functions lie. So ideally, the more basis functions used in a calculation, the better the representation of the unknown orbital will be. However, ab initio calculations increase with the size of the basis set to at least $\mathrm{M}_{\text {basis }}^{4}$ (generally, but can be linearly scaled to $\mathrm{M}_{\text {basis }}^{2}$ ). In other 
words, increasing the size of basis set to get a better representation would result in a very high computational cost. It is, therefore, important to design basis sets that minimise the number of basis functions without compromising the accuracy.

The smallest number of functions possible in a basis set to describe an atom is called the minimal basis set. For example, the minimal basis set for carbon is two $s$-functions to describe the $1 s$ and $2 s$ orbitals of the atom and a set of $p$-functions to describe the $2 p$ orbitals. However, the minimal basis set is too small to describe the orbitals accurately. Therefore, the next improvement over the minimal basis set is to use twice the number of basis functions, producing a double- $\zeta$ (Double Zeta, DZ) basis set. As an example, the second row elements would have four $s$-functions $\left(1 s, 1 s^{\prime}, 2 s\right.$ and $\left.2 s^{\prime}\right)$ and two sets of $\mathrm{p}$-functions ( $2 p$ and $2 p^{\prime}$ - one set of $p$-functions consists of $p_{\mathrm{x}}, p_{\mathrm{y}}$ and $p_{\mathrm{z}}$ functions) in the basis set. The improvement of DZ basis set from minimal basis set can be seen when describing bonding in the $\mathrm{HCN}$ molecule. The $\mathrm{H}-\mathrm{C} \sigma$-bond is primarily formed from the overlap of the $s$-orbital of hydrogen and the $p_{\mathrm{z}}$-orbital of carbon. The $\pi$-bonds between $\mathrm{C}$ and $\mathrm{N}$ atoms, on the other hand, consist of the $p_{\mathrm{x}}$ (and $p_{\mathrm{y}}$ ) orbitals of carbon and nitrogen. The two types of bonding in $\mathrm{HCN}$ molecule both require $p$-functions. If only the minimal basis set is used, the $p$-functions would have to compromise between the two types of bonding. This is where the extra $p$-functions in DZ basis sets come into play to describe the different type of bonding in the molecule. A tighter $p$-function with larger exponent is used to represent $\sigma$-bond where the electrons are more localised in the bond, while a more diffuse function with smaller exponent describes the $\pi$-bonds. Chemical bonds in molecules are generally described by the valence orbitals and using twice the number of basis functions describing core orbitals normally does not produce a significant improvement. Therefore, another type of DZ basis set is used only for increasing the size of valence functions which is called double- $\zeta$ split valence basis set (VDZ). A further improvement to double- $\zeta$ basis sets is to increase the size of the basis sets to triple-, quadruple- or quintuple- $\zeta$. 
The representation of chemical bonding can also be improved by adding higher angular momentum functions, which are known as polarization functions, into the basis set. Considering the $\mathrm{O}-\mathrm{H}$ bond in the water molecule, the electrons are more distributed towards the oxygen atom since it is more electronegative than the hydrogen atom. However, using only $s$-functions for an $\mathrm{H}$ atom one is not able to describe this property at all. This is because an $s$-function is perfectly spherical and it would mean that the electron distribution around $\mathrm{H}$ atoms is evenly distributed, which is incorrect. To solve this problem, a set of $p$-functions is added to describe the $\mathrm{H}$ atom and the $p_{\mathrm{z}}$ component can be used to describe the polarization towards the $\mathrm{O}$ atom. Besides polarization functions, diffuse functions are also often added to basis sets to improve the bonding description in a molecule. Diffuse functions have very small exponents which allow a better description of the 'tail' portion of atomic orbitals more accurately, which are at large distances from the atomic nuclei. These functions are thus useful in describing weak interactions in a system such as hydrogen bonding. They are also important in describing barely bound electrons in anions, i.e. Rydberg states.

There are many types of basis sets to choose from and the selection of these basis sets has to be done carefully to achieve a balance between computational efficiency and accuracy. The basis set that will be used in this study belongs to the family of correlation-consistent basis sets developed by Dunning and coworkers. The correlation-consistent in this context refers to the design of the basis set such that functions that contribute the similar amount of correlation energy are incorporated at the same stage, independent of the function type. The basis sets are denoted as cc-pVnZ where $n$ stands for double(D), triple(T), quadruple(Q), quintuple(5), sextuple(6) and so on. There are augmented versions of these basis sets which are denoted as aug- in the acronym and these basis sets include diffuse functions. The basis set used in this study is of aug-cc-pVTZ quality which indicates that the functions are correlation-consistent polarized with triple- $\zeta$ split valence orbitals and with additional diffuse functions. Gold atoms consist of a relatively large number of electrons (79 electrons) and it requires a large amount of computational time to consider 
the interaction between so many electrons. In addition to that, the relativistic effects of gold has to be included for the results to be reliable. One way to simultaneously reduce the computational cost and include relativistic effects of gold in the calculation is to adopt pseudopotential (or effective core potential) in the basis set. Since the core electrons do not take part in chemical reactions and only the valence electrons show chemically interesting properties, the computational efficiency can be significantly enhanced by using pseudopotentials in the basis set. The pseudopotential used in the present work describes the effective potential of the 60 core electrons in the gold atom and it includes scalar relativistic effects which are important in predicting the behaviour of the gold catalyst accurately. In summary, the basis set used in this project (obtained from EMSL basis set database ${ }^{134,135}$ ) is the correlation consistent triple- $\zeta$ basis sets (aug-cc-pVTZ) for $\mathrm{H}$, $\mathrm{C}, \mathrm{O}^{136}$ and $\mathrm{Cl}^{137}$ atoms while an energy-consistent small-core pseudopotential of the Stuttgart group ${ }^{138}$ is included in the basis set (aug-cc-pVTZ-PP) for the Au atom. ${ }^{139}$

\subsection{Calculation algorithms and methods}

All calculations were done using the Gaussian09 software package ${ }^{140}$ and the results were visualized with GaussView 5. Most problems in computational chemistry can be formulated as an optimisation problem. In computational chemistry, optimisation is a process of finding stationary points on a potential energy surface. In order to locate the stationary points in the energy profile, an approximate starting structure is constructed using Gauss View to specify the coordinates of all the atoms in the molecules and it is then optimised to a stationary point using the chosen level of theory. The optimisation

utilises the Berny algorithm developed by Bernhard Schlegel. ${ }^{141}$ In essence, the algorithm predicts a more energetically favourable structure by using the forces acting on the atoms in the molecules and the second derivative matrix (Hessian matrix). An analytical Hessian matrix is generally preferred in optimisations but the calculation of an accurate Hessian 
matrix is very expensive especially if it is done in every single optimisation step. In most cases, the algorithm processes an estimated Hessian matrix at the beginning of the calculation and updates it by utilising the energies and first derivatives calculated along the optimisation steps. To ensure a good initial guess of the Hessian matrix, the matrix can be calculated analytically in the first step of the optimisation or read in from a previous calculation with a smaller basis set. If a minimum point in the potential energy surface is desired, the algorithm first performs a linear search between the latest point and the previous point having the lowest energy. A polynomial function (usually quintic or quartic) is then fitted to the energies and first derivatives with the condition that the second derivative of the polynomial is more than zero to ensure that it has one minimum. Using the updated Hessian matrix and the results from the linear search, the algorithm gives the next estimate of the stationary point. In Gaussian09, the geometry optimisation utilises energy-represented direct inversion in the iterative subspace, GEDIIS method. ${ }^{142}$ This method searches for a new geometry in the region close to the local potential energy surface through interpolation only. The enforced interpolation constraint enhances the stability of the optimisation by avoiding erroneously large extrapolations away from the optimised geometry in the case when the molecular geometry is far from converged. Lastly, the algorithm tests the gradient and displacement vectors in each step to check if the optimisation has converged. Convergence is achieved when the root-mean-square of the gradient, the maximum gradient component, the root-mean-square of the displacement and the maximum displacement vector fall below a certain threshold specified in the calculation.

In many cases, the desired stationary point is a minimum where all eigenvalues in the Hessian matrix are positive but it is also possible for optimisations to locate a higher order saddle point. The study of reaction mechanisms often involves the search of multiple elementary steps that each is governed by a transition state which is first-order saddle point, having exactly one negative eigenvalue in the Hessian matrix. Transition state structures were obtained using a guessed starting structure as well but they were optimised 
to a saddle point along the reaction coordinate. The nature of all stationary points in

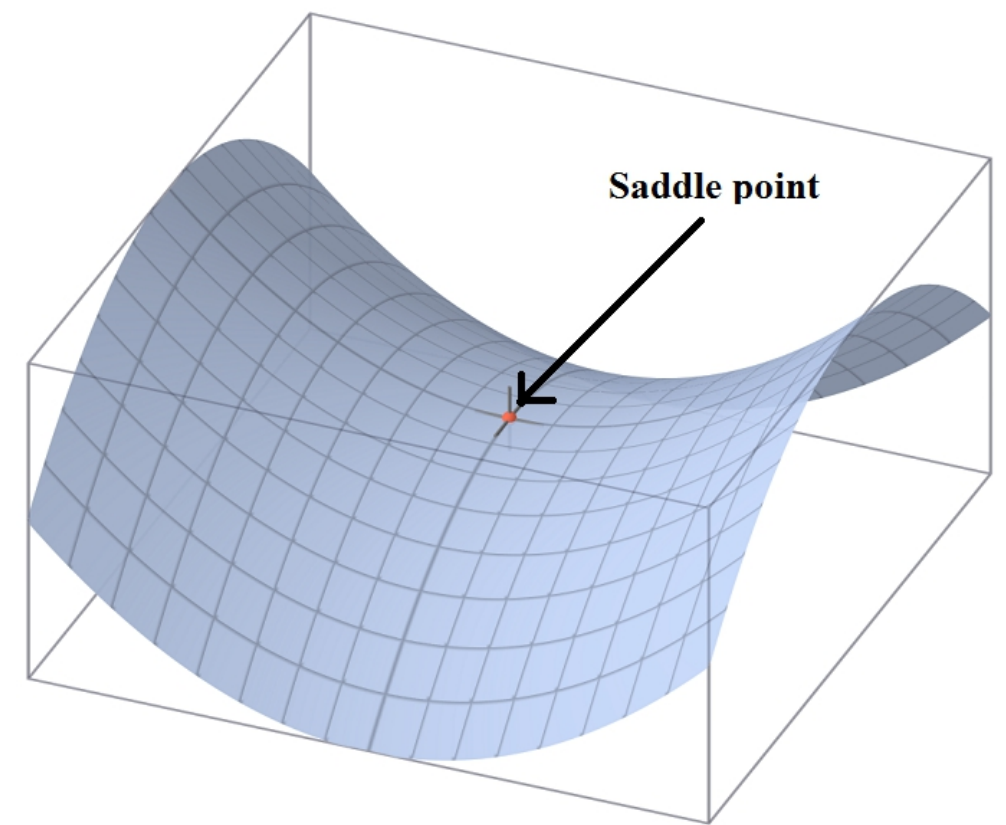

Figure 2.4 Location of a saddle point in the energy profile.

the energy profile of the nucleophilic addition of methanol to alkynes were determined through the calculation of the Hessian matrix. In a minimum, all eigenvalues of the Hessian matrix are positive which means that all vibrational frequencies calculated are real numbers while the transition states have one imaginary frequency.

Once a transition state is located, it must be verified that the transition state indeed leads to the desired product. A non-linear transition state has $3 N-7$ vibrational modes and one imaginary vibration that corresponds to the motion of atom(s) that is involved when the reaction occurs. As an example, for an $\mathrm{S}_{\mathrm{N}}{ }^{2}$ nucleophilic substitution reaction, the negative vibrational mode corresponds to the asymmetric stretching of the approaching nucleophile and the leaving group with the central atom. By following the vibrational coordinate associated with the imaginary frequency, one can determine the pathway towards the connecting minima which are the reactants and products for that particular step. For this purpose, intrinsic reaction coordinate (IRC) calculations were employed to locate the minima connected by the corresponding transition state. Points on the IRC path can be 
located by steepest descent downhill from the previously optimized transition state in the potential energy surface to the reactant and product wells.

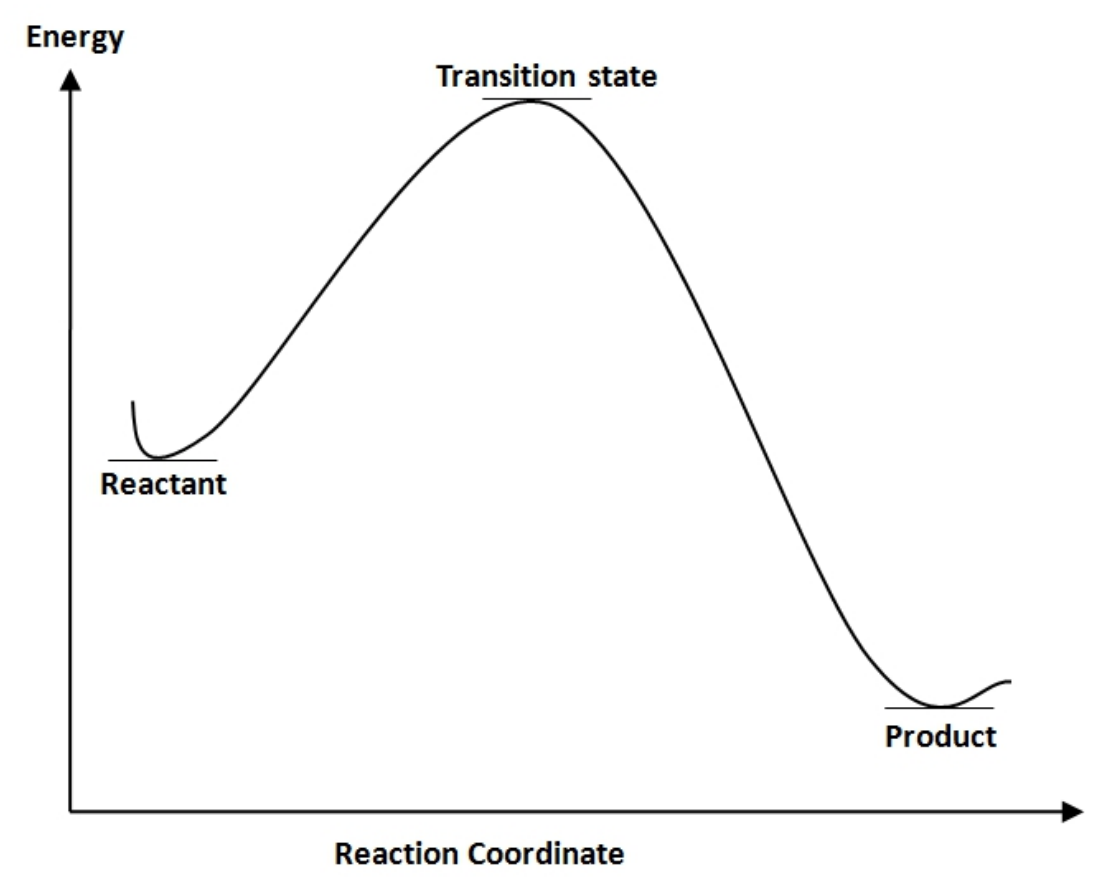

Figure 2.5 A typical energy profile along the reaction coordinate.

The algorithm in Gaussian09 makes use of a Hessian based predictor-corrector integrator to locate points along the reaction path. Local quadratic approximation (LQA) is used for the predictor step while a modified Bulirsch-Stoer algorithm is used for the corrector step. ${ }^{143,144}$ However, the last point in the IRC path may or may not be the local minimum as it is possible for an optimisation to move across a flat energy surface before it reaches the local minimum region. In order to ensure convergence to the local minimum, the last point in the IRC path was used as the initial geometry for an optimisation calculation. By doing that, the path is extended to ensure that it reaches the potential well that is the local minimum. A complex reaction normally involves many steps before producing the final product and this means that there will be many transition states. An overall energy profile is constructed by connecting a number of sets of IRC results accordingly to represent the mechanism of the reaction under study.

In some mechanistic studies, the calculations are done in solution phase environment 
to better represent the energy profile of the reaction. In this method, the solvent is represented by a homogeneous continuum environment which is polarized by the solute located in a cavity within the sea of solvents. The interactions with the solvent are represented as a reaction potential, $\mathbf{V}_{\mathrm{R}}$ which acts as a perturbation on the solute Hamiltonian as follows.

$$
\begin{gathered}
\mathbf{H}^{o} \psi=E^{o} \psi \quad \text { in gas phase } \\
{\left[\mathbf{H}^{o}+\mathbf{V}_{\mathrm{R}}\right] \psi=E \psi \quad \text { in solution phase. }}
\end{gathered}
$$

These calculations are mostly done in the Polarizable Continuum Model (PCM) using the integral equation formalism (IEF-PCM). ${ }^{145,146}$ Within the PCM framework, the reaction potential is described as a set of induced point charges located at the centre of small surface elements covering the cavity in the dielectric medium, where the solute can be found. This method, however, does not cause much changes to the reaction energy profile when both the solute and solvent are neutrally charged. This is demonstrated in Lein's work on gold-catalysed hydration reaction where the solution phase energy profile is qualitatively similar to the gas phase energy profile. ${ }^{117}$ On top of that, the PCM method is unable to account for any explicit interactions between the solvent and solute molecules that might be observed in the reaction processes. Therefore, in the current study, solvent molecules (methanol) are explicitly included in the system to account for such interactions and to provide a clear observation of how these interactions affect the energy profile of the reaction.

\subsection{Transition State Theory and reaction kinetics}

The Transition State Theory (TST) is a semi-classical theory that is used to understand how chemical reactions occur whereby the dynamics along the reaction coordinate is treated classically but the quantum nature is included through quantization of vibrational 
and rotational energy states. TST postulates that the rate of reaction is primarily determined by the rate of which an activated complex goes through a transition state, which is a saddle point on a potential energy surface. The activated complex is assumed to be in a quasi-equilibrium with the reactants. Products can be formed if the activated complex possesses enough kinetic energy to overcome an energy barrier of the reaction. It is assumed that the particles behave classically and collide with each other to gather enough energy to form the transition state that proceeds to forming products in the potential energy surface. Most experiments deal with large sample (macroscopic) while calculations often only involve relatively few particles (microscopic). A connection between the properties of macroscopic and microscopic systems can be provided by statistical mechanics - a branch in chemistry to study the average behaviour of a mechanical system using probability theory. A key feature in statistical mechanics is the partition function, $q$ which allows for the calculations of all macroscopic functions. The partition function can be written as a sum over all energy levels, multiplied with a degeneracy factor $g_{i}$ which indicates the number of states with the same energy $\varepsilon_{i}$,

$$
q=\sum_{i=\text { states }}^{\infty} g_{i} e^{-\varepsilon_{i} / k_{B} T}
$$

where $k_{B}$ is the Boltzmann's constant and $\mathrm{T}$ is the temperature $(298 \mathrm{~K})$. The calculation of partition function requires one to know all possible quantum states for a system and such rigorous approach is only possible for small system. For an isolated polyatomic molecule, the energy levels of a specific conformation can be calculated under the rigidrotor harmonic-oscillator approximation, where the translational, rotational, vibrational and electronic degree of freedoms are separable. An isolated molecule is of an ideal gas state and hence the partition function can be calculated exactly in the aforementioned approximation. The total partition function is made up of the product of the translational, rotational, vibrational and electronic partition functions.

$$
q=q_{\text {trans }} q_{\text {rot }} q_{\text {vib }} q_{\text {elec }}
$$




\subsubsection{Translational partition functions}

The quantum states of translational energy of a particular molecule can be determined by using a three-dimensional 'particle in a box' model which has a zero potential within the box itself but infinite potential outside the box. The solutions to the Schrödinger equation for this model are mostly sine and cosine functions. The total energy levels, $E_{n_{x} n_{y} n_{z}}$ in three-dimensional is the sum of the corresponding energy level in each direction, $\varepsilon_{n_{x}}, \varepsilon_{n_{y}}$ and $\varepsilon_{n_{z}}$ given by Equation 2.9.

$$
\begin{gathered}
\varepsilon_{n_{x}}=\frac{n_{x}^{2} h^{2}}{8 a^{2} m} \quad \varepsilon_{n_{y}}=\frac{n_{y}^{2} h^{2}}{8 b^{2} m} \quad \varepsilon_{n_{z}}=\frac{n_{z}^{2} h^{2}}{8 c^{2} m} \\
E_{n_{x} n_{y} n_{z}}=\frac{h^{2}}{8 M}\left(\frac{n_{x}^{2}}{a^{2}}+\frac{n_{y}^{2}}{b^{2}}+\frac{n_{z}^{2}}{c^{2}}\right)
\end{gathered}
$$

where, $\quad V=a \times b \times c=$ volume of the box

The energy levels are quantized but the energy difference between the levels is so small that it can be treated as continuous. The summation involved in the calculation of partition function can therefore be represented as an integral. Inserting the energy expression into the integral and performing the integration, the expression for $q_{\text {trans }}$ can be derived as follows,

$$
\begin{aligned}
q_{x}=\sum_{n_{x}=0}^{\infty} e^{-\varepsilon_{n_{x}} / k_{B} T} & \approx \int_{0}^{\infty} e^{-\varepsilon_{n_{x}} / k_{B} T} \mathrm{~d} n_{x} \\
q_{\text {trans }} & =q_{x} q_{y} q_{z} \\
q_{\text {trans }} & =\frac{V}{\Lambda^{3}} \\
\text { where, } \quad \Lambda & =\frac{h}{\sqrt{2 \pi m k_{B} T}}
\end{aligned}
$$

where, $m$ is the mass of a molecule, and $h$ is the Planck's constant. 


\subsubsection{Rotational partition functions}

In order to calculate the rotational partition function, we assume the rigid rotor approximation for the system. Within the rigid rotor approximation, the rotation of a molecule is assumed to occur in a fixed geometry. The simplest case for this model would be a linear molecule and the energy levels calculated from the Schrödinger equation are given in Equation 2.15,

$$
\begin{gathered}
\varepsilon_{J}=J(J+1) \frac{h^{2}}{8 \pi^{2} I} \\
I=\sum_{i=1}^{N} m_{i} d_{i}^{2}
\end{gathered}
$$

where $J$ is the rotational quantum number, $I$ is the moment of inertia, $m_{i}$ is the mass of atom $i$ and $d_{i}$ is the distance of atom $i$ from the centre of mass of the molecule.

Similar to the translational energy, the spacing between the rotational energy levels is very small compared to $k_{B} T$ and the summation in the calculation of partition function can thus be treated as an integral. Inserting the energy expression into the integral and performing the integration yields the expression of $q_{\text {rot }}$ for linear molecules (Equation 2.18).

$$
\begin{gathered}
q_{\text {rot }}=\sum_{J=0}^{\infty} e^{-\varepsilon_{J} / k_{B} T} \approx \int_{0}^{\infty} e^{-\varepsilon_{J} / k_{B} T} \mathrm{~d} J \\
q_{\text {rot }}=\frac{8 \pi^{2} I k_{B} T}{h^{2} \sigma}
\end{gathered}
$$

When the system is a non-linear polyatomic molecule (which is the case in this project), the calculation for the the moment of inertia becomes much complicated. The moment of inertia for such system is expressed in a $3 \times 3$ matrix.

$$
I=\left(\begin{array}{ccc}
\sum_{i} m_{i}\left(y_{i}^{2}+z_{i}^{2}\right) & -\sum_{i} m_{i} x_{i} y_{i} & -\sum_{i} m_{i} x_{i} z_{i} \\
-\sum_{i} m_{i} x_{i} y_{i} & \sum_{i} m_{i}\left(x_{i}^{2}+z_{i}^{2}\right) & -\sum_{i} m_{i} y_{i} z_{i} \\
-\sum_{i} m_{i} x_{i} z_{i} & -\sum_{i} m_{i} y_{i} z_{i} & \sum_{i} m_{i}\left(x_{i}^{2}+y_{i}^{2}\right)
\end{array}\right)
$$


The matrix can be diagonalized with the eigenvalues being the moments of inertia for rotation about the three axes and the eigenvectors are the principal axes of inertia. The rotational energy levels for a non-linear polyatomic molecule cannot be expressed in a simple form. A good approximation from classical mechanics is used and hence the rotational partition function, $q_{\mathrm{rot}}$ is derived,

$$
q_{\mathrm{rot}}=\frac{\pi^{1 / 2}}{\sigma}\left(\frac{8 \pi^{2} I_{x} k_{B} T}{h^{2}}\right)^{1 / 2}\left(\frac{8 \pi^{2} I_{y} k_{B} T}{h^{2}}\right)^{1 / 2}\left(\frac{8 \pi^{2} I_{z} k_{B} T}{h^{2}}\right)^{1 / 2}
$$

where, $I_{x, y, z}$ is the moment of inertia for rotation about the corresponding axis $(x, y$ or $z)$ and $\sigma$ is the rotational symmetry.

\subsubsection{Vibrational partition function}

In the lowest approximation, the molecular vibrations are assumed to follow those of a harmonic oscillator - a system that experiences a restoring force proportional to the displacement from the equilibrium position. We consider the simplest case of a diatomic molecule and the energy of each energy level calculated from the Schrödinger equation is expressed as,

$$
\varepsilon_{n}=\left(n+\frac{1}{2}\right) h v
$$

where, $v$ is the vibrational frequency of the diatomic molecule, which is dependent on the force constant and mass of the system. In contrast to the translational and rotational energy levels, the spacing between the vibrational energy levels is comparable to $k_{B} T$ at temperature $298 \mathrm{~K}$ and thus the summation in the expression of partition function cannot be treated as an integral.

$$
\begin{gathered}
q_{\mathrm{vib}}=\sum_{n=0}^{\infty} e^{-\varepsilon_{n} / k_{B} T}=e^{-h v / 2 k_{B} T}+e^{-3 h v / 2 k_{B} T}+e^{-5 h v / 2 k_{B} T}+\cdots \\
q_{\mathrm{vib}}=e^{-h v / 2 k_{B} T}\left(1+e^{-h v / k_{B} T}+e^{-2 h v / k_{B} T}+\cdots\right)
\end{gathered}
$$




$$
q_{\mathrm{vib}}=\frac{e^{-h v / 2 k_{B} T}}{1-e^{-h v / k_{B} T}}
$$

Taking the zero point energies as reference points, $q_{\text {vib }}$ for a diatomic molecule is expressed as shown.

$$
q_{\mathrm{vib}}=\frac{1}{1-e^{-h v / k_{B} T}}
$$

For a polyatomic molecule, it becomes very difficult to describe the molecular vibrations mathematically. However, a set of coordinates can be chosen so that each vibrational mode is considered to be independent of the others. This is called the normal coordinates. Thus, the vibrational energy levels in polyatomic molecules are expressed as a sum over all vibrational modes of the molecule.

$$
E_{\mathrm{vib}}=\sum_{i=1}^{n_{\mathrm{vib}}}\left(n_{i}+\frac{1}{2}\right) h v_{i}
$$

where, $n_{\mathrm{vib}}$ is the number of vibrational modes in a molecule ( $3 N-5$ for linear molecule and $3 N-6$ for non-linear molecule) and $v_{i}$ is the vibrational frequency of the $i^{\text {th }}$ vibrational mode. Since the energy can be expressed as a sum, the partition function (based on the diatomic expression) can be written as the product of all $n_{\text {vib }}$ vibrational partition functions.

$$
q_{v i b}=\prod_{i=1}^{n_{\mathrm{vib}}} \frac{1}{1-e^{-h v_{i} / k_{B} T}}
$$

\subsubsection{Electronic partition function}

The electronic partition function involves a sum over electronic quantum states which are the solutions for Schrödinger equation. These are the lowest (ground) state and all possible excited states. in general, the energy difference between the ground state and excited states is large compared to $k_{B} T$ at temperature $298 \mathrm{~K}$. As a result, only the first term of the partition function summation is considered important and the subsequent terms 
becomes negligible.

$$
q_{\text {elec }}=\sum_{i=0}^{\infty} g_{i} e^{-\varepsilon_{i} / k_{B} T}=g_{0} e^{-\varepsilon_{0} / k_{B} T}+g_{1} e^{-\varepsilon_{1} / k_{B} T}+\cdots \approx g_{0} e^{-\varepsilon_{0} / k_{B} T}
$$

If we define the reference point for the energy as the electronic energy of the reactant, the electronic partition functions for reactant and the transition state (TS) are as follows,

$$
\begin{gathered}
q_{\text {elec }}^{\text {react }}=g_{0} \\
q_{\text {elec }}^{\mathrm{TS}}=g_{0} e^{-\Delta E^{\ddagger} / k_{B} T}
\end{gathered}
$$

where, $\Delta E^{\ddagger}$ is the activation energy for the reaction and $g_{0}$ is usually 1 (non-degenerate).

\subsubsection{Rate constant}

Under these assumptions in TST, the expression to calculate the rate constant of individual elementary steps is as given in Equation 2.31, ${ }^{147}$

$$
k=\frac{k_{B} T}{h} \frac{q_{\mathrm{TS}}}{\prod q_{\text {react }}}
$$

We can further decompose Equation 2.31 into electronic and nuclear (translational, rotational and vibrational) partition functions to give,

$$
\begin{gathered}
k=\frac{k_{B} T}{h} \frac{q_{\mathrm{TS}}^{\text {nuc }}}{\prod q_{\mathrm{react}}^{\text {nuc }}} \frac{q_{\mathrm{TS}}^{\text {elec }}}{\prod q_{\mathrm{react}}^{\text {elec }}} \\
k=\frac{k_{B} T}{h} \frac{q_{\mathrm{TS}}^{\text {nuc }}}{\prod q_{\mathrm{react}}^{\text {nuc }}} e^{-\frac{E_{\mathrm{TS}}-\sum_{\text {react }}}{k_{B} T}}
\end{gathered}
$$

where $k$ is rate constant, $k_{B}$ is the Boltzmann's constant, $T$ is the temperature, $h$ is the Planck's constant, $q_{\mathrm{TS}}$ is the total partition function of transition state, $q_{\text {react }}$ is the total 
partition function of the reactant species, $E_{\mathrm{TS}}$ is the energy of transition state and $E_{\text {react }}$ is the energy of reactant.

Knowing the rate constants of each elementary step, the concentrations of each species throughout the mechanism can be calculated at any given time by solving the equations for the rate of each step simulataneously.

Considering a reaction of $\mathrm{A}+\mathrm{B} \stackrel{\mathrm{k}_{1}}{\rightarrow} \mathrm{C}$ and $\mathrm{C} \stackrel{\mathrm{k}_{2}}{\rightarrow} \mathrm{D}$, the rate of reactions can be expressed as:

$$
\begin{gathered}
\frac{\mathrm{d}[\mathrm{A}]}{\mathrm{d} t}=-k_{1}[\mathrm{~A}][\mathrm{B}] \\
\frac{\mathrm{d}[\mathrm{B}]}{\mathrm{d} t}=-k_{1}[\mathrm{~A}][\mathrm{B}] \\
\frac{\mathrm{d}[\mathrm{C}]}{\mathrm{d} t}=k_{1}[\mathrm{~A}][\mathrm{B}]-k_{2}[\mathrm{C}] \\
\frac{\mathrm{d}[\mathrm{D}]}{\mathrm{d} t}=k_{2}[\mathrm{C}]
\end{gathered}
$$

and the concentrations at any given time can be obtained by solving these equations simultaneously which will be further elaborated in Chapter 6.3. 


\section{Chapter 3}

\section{Results and Discussions (Reaction 1):}

\section{Mechanism of the Reaction of Propyne with Methanol Catalysed by $\mathrm{AuCl}_{3}$}

The reaction discussed in this section is given as follows:

$$
\mathrm{HC} \equiv \mathrm{CCH}_{3}+2 \mathrm{CH}_{3} \mathrm{OH} \stackrel{+\mathrm{AuCl}_{3}}{\longrightarrow} \mathrm{CH}_{3} \mathrm{C}\left(\mathrm{OCH}_{3}\right)_{2} \mathrm{CH}_{3}
$$

The goal in this chapter is to investigate the reaction mechanism of hydroalkoxylation of propyne and to compare it with the hydration reaction mechanism. The results are reported in two functionals, BP86 and PBE0. With PBE0 as the main functional, the values calculated by BP86 are reported in parentheses in the figures shown. The comparison between this mechanism and the one done in the past ${ }^{117}$ with water as nucleophile will be discussed based on BP86 results at the end of the section.

\subsection{Reaction sequence}

In the first step of the reaction, the $\mathrm{AuCl}_{3}$ catalyst $\left(\mathbf{1}_{\mathrm{s}}\right)$ coordinates to the propyne substrate. Two conformations were found for this activated complex due to the small rotational barrier about the $\mathrm{C}-\mathrm{Au}$ bond. The two conformations are shown in Figure 3.1 with $\mathrm{AuCl}_{3}$ having a perpendicular $\left(\mathbf{2}_{\mathrm{s}}^{\prime}\right)$ and in-plane $\left(\boldsymbol{2}_{\mathrm{s}}\right)$ orientation with respect to propyne. The in-plane orientation is energetically more favourable by $5.2 \mathrm{kcal} \mathrm{mol}^{-1}$. Structure $2_{\mathrm{s}}$ is stabilised by $37.9 \mathrm{kcal} \mathrm{mol}^{-1}$ with respect to $\mathrm{AuCl}_{3}$ and propyne. It should be noted that since the nucleophile has not yet attacked the activated complex, this 
structure is identical to the one found by Lein in a previous study where the nucleophile under consideration was water. ${ }^{117}$ In analogy to these previous results, the oxygennucleophile forms two hydrogen bonds with the activated complex $\boldsymbol{2}_{\mathrm{s}}$ to form $\mathbf{3}_{\mathrm{s}}$. In $\mathbf{3}_{\mathrm{s}}$, the methanol oxygen atom coordinates to the terminal hydrogen atom of propyne, while the methanol-OH-proton forms a hydrogen bond with one of the catalyst's chlorine atoms. This addition of methanol and the formation of two hydrogen bonds further stabilises the system by $5.4 \mathrm{kcal} \mathrm{mol}^{-1}$.
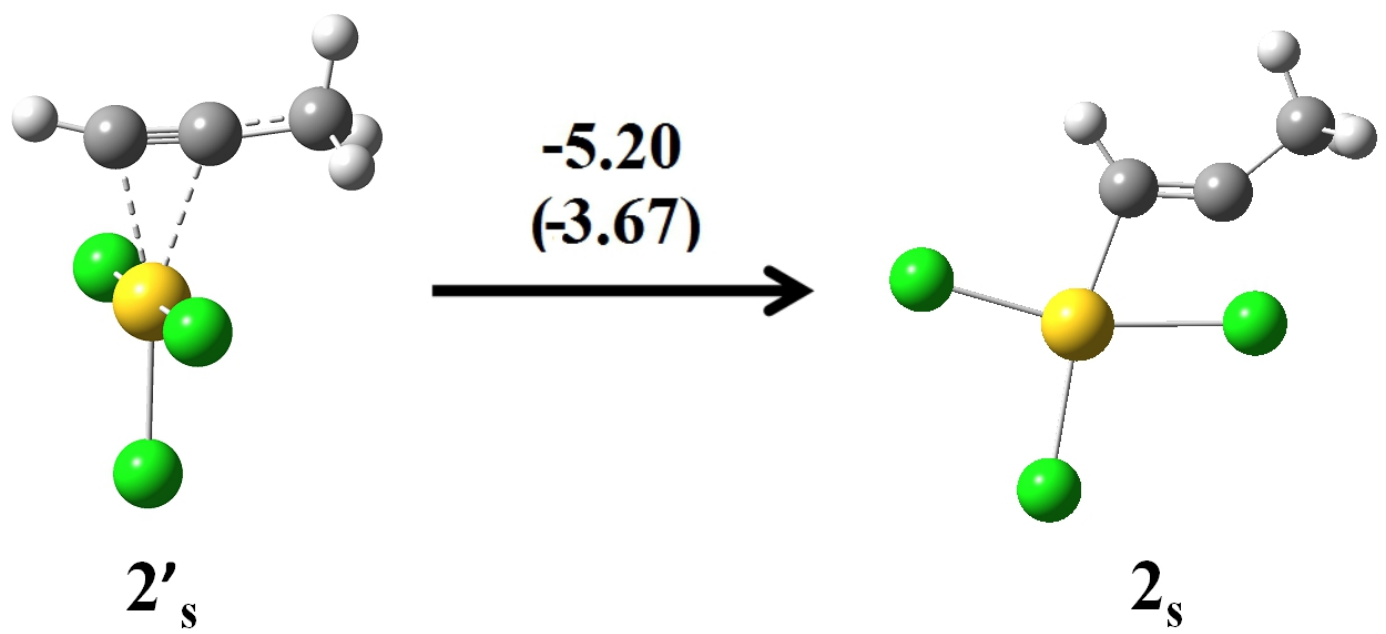

Figure 3.1 The perpendicular, $\mathbf{2}_{\mathrm{S}}^{\prime}$ and in-plane, $\boldsymbol{2}_{\mathrm{s}}$ conformations of $\mathrm{AuCl}_{3}$-propyne activated complex. All energetic changes from PBE0 and BP86 calculations are given in $\mathrm{kcal} \mathrm{mol}^{-1}$. Values for BP86 calculations are given in parentheses.

Next is the addition of a second methanol molecule which leads to an extension of the hydrogen bonding network. There are two possibilities where the methanol can be inserted, causing a split of the reaction pathway. The second methanol molecule can either insert itself between the catalyst and the first methanol molecule to give $\mathbf{4}_{\mathrm{s}}$, or between the first methanol molecule and the propyne substrate to give $\mathbf{4}_{\mathrm{s}}^{\prime}$. When $\mathbf{4}_{\mathrm{s}}$ and $\mathbf{4}_{\mathrm{s}}^{\prime}$ are compared, it becomes immediately obvious that only $\mathbf{4}_{\mathrm{s}}^{\prime}$ has a nucleophilic oxygen atom in close proximity $(2.34 \AA$ ) to a possible site of attack, while the reaction cannot proceed out of $\mathbf{4}_{\mathrm{s}}$ in the same fashion. 


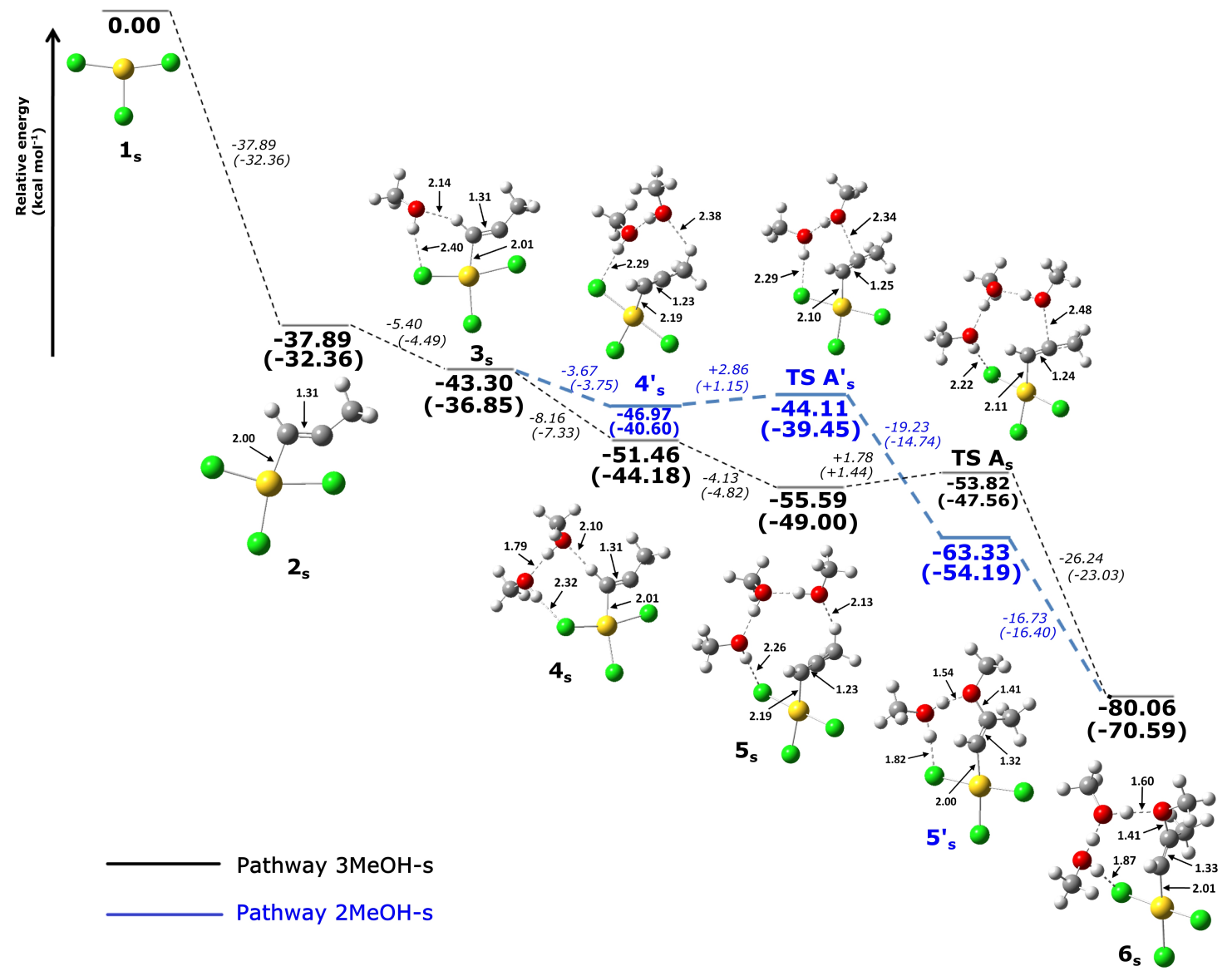

Figure 3.2 First part of the mechanism. Relative energies with respect to the starting materials of PBE0 calculations are given below structures $\mathbf{1}_{\mathrm{s}}$ to $\mathbf{6}_{\mathrm{s}}$ respectively while energetic changes with respect to the previous structures are given on the dotted lines that represent the reaction coordinate. Values for BP86 calculations are given in parentheses. The structural parameters are in $\AA$. All energies in $\mathrm{kcal} \mathrm{mol}^{-1}$.

\subsubsection{Pathway 3MeOH-s}

Because $\mathbf{4}_{\mathrm{s}}$ is not reactive with respect to nucleophilic addition to the propyne substrate, the addition of a third methanol molecule is necessary to yield $\mathbf{5}_{\mathrm{s}}$ which is now in a position to proceed with a nucleophilic attack. The activation barrier for the TS $\mathbf{A}_{\mathrm{s}}$ is $1.8 \mathrm{kcal} \mathrm{mol}^{-1}$ and the reaction yields the addition product $\mathbf{6}_{\mathrm{s}}$, which is substantially stabilised by $26.2 \mathrm{kcal} \mathrm{mol}^{-1}$ with respect to the transition state. As the reaction proceeds from $\mathbf{5}_{\mathrm{s}}$ to $\mathbf{6}_{\mathrm{s}}$, the distance between the attacking oxygen atom and the central carbon atom is found to be $2.48 \AA$ in $\mathbf{T S} \mathbf{A}_{\mathrm{s}}$ and decreases to $1.41 \AA$ when the product is formed. An 
increase from $1.24 \AA$ to $1.33 \AA$ in the $\mathrm{C}-\mathrm{C}$ multiple bond of the substrate was also seen. $\mathbf{6}_{\mathrm{s}}$ is an interesting intermediate. Firstly, it represents the point where the two branches of the reaction mechanism converge again. Secondly, and more importantly, it represents the stage of the mechanism where the initial nucleophilic attack has taken place, but the acidic $\mathrm{OH}$-proton of the attacking methanol has not migrated to the substrate yet.

\subsubsection{Pathway 2MeOH-s}

In contrast to the nucleophilic attack discussed in the previous section, the second methanol molecule can proceed with a nucleophilic attack straight after the formation of $\mathbf{4}_{\mathrm{s}}^{\prime}\left(\mathbf{4}_{\mathrm{s}}^{\prime}\right.$, $\mathbf{T S} \mathbf{A}_{\mathrm{s}}^{\prime}$ and $\mathbf{5}_{\mathrm{s}}^{\prime}$ in Figure 3.2 are highlighted in blue). The activation barrier for this process is also very low $\left(2.9 \mathrm{kcal} \mathrm{mol}^{-1}\right)$. The product of the nucleophilic attack $\mathbf{5}_{\mathrm{s}}^{\prime}$ is structurally similar to product $\mathbf{6}_{\mathrm{s}}$ of the nucleophilic attack discussed previously. However, hydrogen migration does not take place out of $\mathbf{5}_{\mathrm{s}}^{\prime}$. Hence, a third molecule of methanol is required to facilitate this last step of the first nucleophilic addition. This leads to the convergence of the two possible reactions paths at structure $\mathbf{6}_{\mathrm{s}}$.

\subsubsection{Hydrogen migration}

Hydrogen migration was found to proceed via two pathways. In the first pathway, the attacking methanol was deprotonated by the neighbouring solvent methanol and the two solvent molecules rearrange themselves to allow for the migrating hydrogen to be in close proximity $(1.77 \AA)$ to the terminal carbon atom for the migration to happen $\left(\mathbf{6} \mathbf{a}_{\mathrm{s}}\right)$. The new arrangement of solvent molecules was found to be $1.4 \mathrm{kcal} \mathrm{mol}^{-1}$ higher in energy. The hydrogen migration then proceeds with an activation barrier of only $1.5 \mathrm{kcal} \mathrm{mol}^{-1}$ to produce the hydrogen migration product $\mathbf{7} \mathbf{a}_{\mathrm{s}}$. The distance between the migrating hydrogen and the terminal carbon atom decreases from $1.77 \AA$ in $\mathbf{6 a}_{\mathrm{s}}$ to $1.52 \AA$ in $\mathbf{T S} \mathbf{B}_{\mathrm{s}}$ 
and finally attaching itself to the terminal carbon atom at $1.10 \AA$ in $\mathbf{7} \mathbf{a}_{\mathrm{s}}$. This is followed by an exothermic rearrangement step of $1.2 \mathrm{kcal} \mathrm{mol}^{-1}$ to form the enol ether $7_{\mathrm{s}}$ as a preparation for the upcoming nucleophilic attack.

The hydrogen migration by the other pathway (highlighted in grey in Figure 3.3) occurs immediately after $\mathbf{6}_{\mathrm{s}}$ without any rearrangement of solvents. The solvent molecules form a hydrogen bonding network $(\mathrm{O} \cdots \mathrm{H} \cdots \mathrm{O} \cdots \mathrm{H} \cdots)$ with the substrate and it becomes clear that the migration can be facilitated by the hydrogen bonds in the system. This network acts as a 'track' for the hydrogens to move from one methanol molecule to another, with the 'track' ending at the position of the terminal carbon atom. Within this network, the hydrogens migrate successfully to form $\mathbf{7}_{\mathrm{s}}^{\prime}$. However, the activation energy required for this step to occur is substantially higher $\left(9.6 \mathrm{kcal} \mathrm{mol}^{-1}\right)$ than the one for the first pathway.

The reason behind this can be seen in the structure of $\mathbf{6}_{\mathrm{s}}$. In $\mathbf{6}_{\mathrm{s}}$, there exists a $\mathrm{H} \cdots \mathrm{Cl}$ interaction which holds the solvent molecules in place. The interaction has to be broken in order to form the hydrogen bonding network that facilitates the hydrogen migration. Energy is required to break this interaction and thus results in a higher activation energy compared to the first migration pathway. The energy profile then goes energetically downwards by $21.8 \mathrm{kcal} \mathrm{mol}^{-1}$ to form structure $7^{\prime}{ }_{\mathrm{s}}$ where the two methanols are seen to roam further away from the substrate since there is no interaction that holds the solvent molecules in place. For the second nucleophilic attack to occur, the interaction is re-established to hold the reactants in place. This is an exothermic process of $2.3 \mathrm{kcal} \mathrm{mol}^{-1}$, forming the enol ether $7_{\mathrm{s}}$, which is the final product of the first nucleophilic attack. The two pathways converge at the formation of $\mathbf{7}_{\mathrm{s}}$. However, the migration would most likely proceed through the first pathway as it requires less activation energy than the second one. 


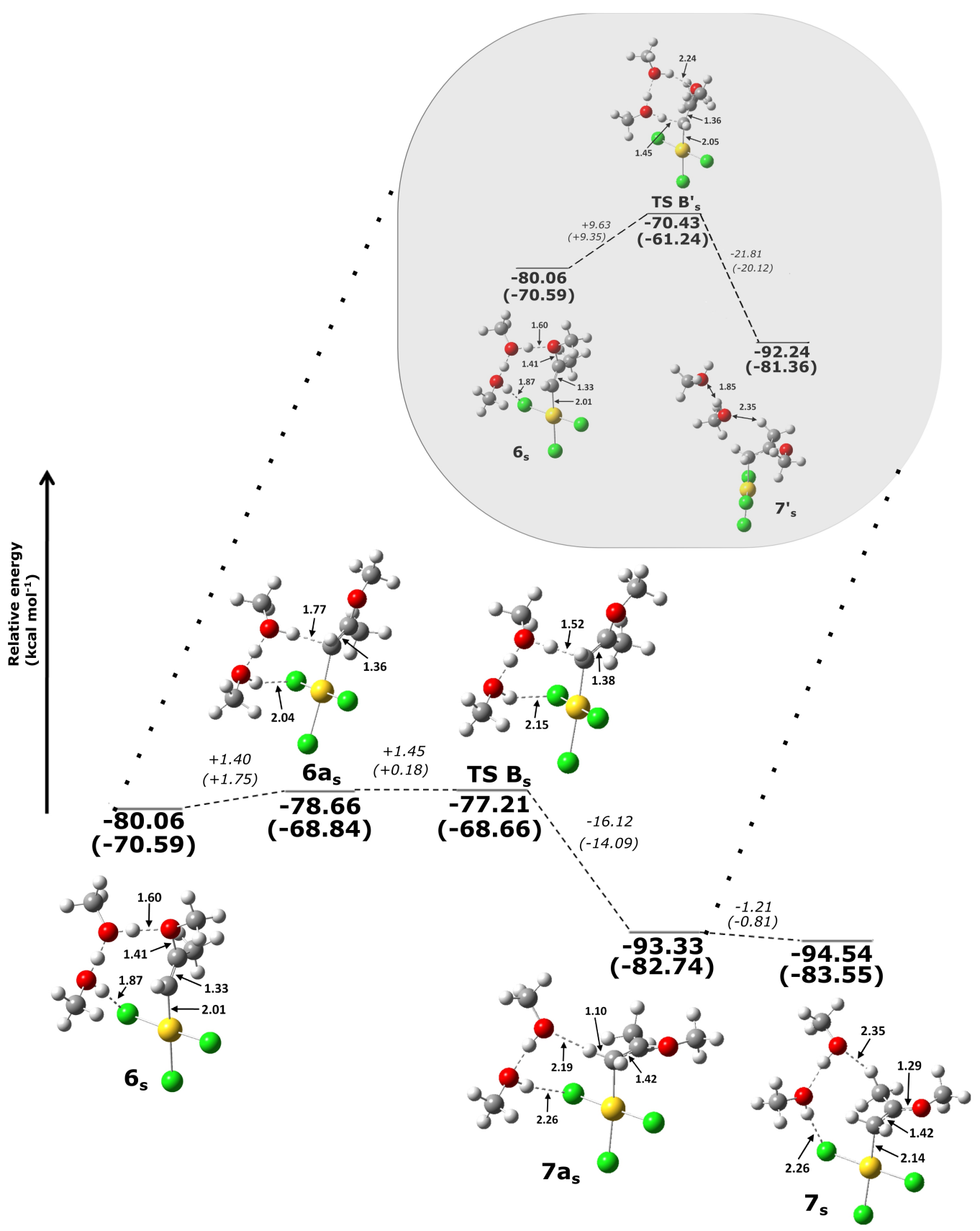

Figure 3.3 Second part of the mechanism. Relative energies with respect to the starting materials of PBE0 calculations are given below structures $\mathbf{6}_{\mathrm{s}}$ to $\mathbf{7}_{\mathrm{S}}$ respectively while energetic changes with respect to the previous structures are given on the dotted lines that represent the reaction coordinate. Values for BP86 calculations are given in parentheses. The structural parameters are in $\AA$. All energies in $\mathrm{kcal} \mathrm{mol}^{-1}$. 


\subsubsection{Second nucleophilic attack}

As can be seen in the structure of $\mathbf{7}_{\mathrm{s}}$, the second methanol molecule is now in a favourable orientation towards a second nucleophilic attack in analogy to Pathway $2 \mathrm{MeOH}-\mathrm{s}$. The transition state (TS $\mathbf{C}_{\mathrm{s}}$ ) for this step is somewhat higher in energy $\left(7.7 \mathrm{kcal} \mathrm{mol}^{-1}\right.$ with respect to reactants) than during the first attack. This is most likely due to the steric hindrance by the methoxy-group introduced to the substrate previously. $\mathbf{T S} \mathbf{C}_{\mathrm{s}}$ is a late transition state that is structurally very similar to product $\mathbf{8}_{\mathrm{s}}$, which is only $4.0 \mathrm{kcal} \mathrm{mol}^{-1}$ lower in energy than $\mathbf{T S} \mathbf{C}_{\mathrm{s}}$ (shown in Figure 3.4). Similarly to the first nucleophilic attack, the nucleophile draws near to the reaction site at about $2.18 \AA$ in $\mathbf{T S} \mathbf{C}_{\mathrm{s}}$ and the distance decreases to $1.45 \AA$ in $\mathbf{8}_{\mathrm{s}}$.

The hydrogen migration that finalises the reaction mechanism requires the largest activation energy (TS $\mathbf{D}_{\mathrm{s}}$ ) of $38.3 \mathrm{kcal} \mathrm{mol}^{-1}$ and leads to ketal $\mathbf{9}_{\mathrm{s}}$, the final product with the additional methanol molecule that facilitated the hydrogen migration in the previous step and the gold catalyst still weakly coordinated ( $2.50 \AA$ from the terminal carbon atom). Rather surprisingly it is this final step of the catalytic cycle that we predict to be rate determining for the reaction. The endothermic nature of this final hydrogen migration also comes as a surprise. In contrast to the enol-form of the ketone that is observed to be the product of the nucleophilic addition of water to propyne, ${ }^{117}$ the ketal that is formed in this reaction has no corresponding enol-form that would allow the gold catalyst to coordinate to. This causes the product $\mathbf{9}_{\mathrm{s}}$ to be more than $20 \mathrm{kcal} \mathrm{mol}^{-1}$ higher in energy than intermediate $\mathbf{8}_{\mathrm{s}}$. The overall most probable mechanism for the reaction between methanol and propyne is depicted in Figure 3.5. 


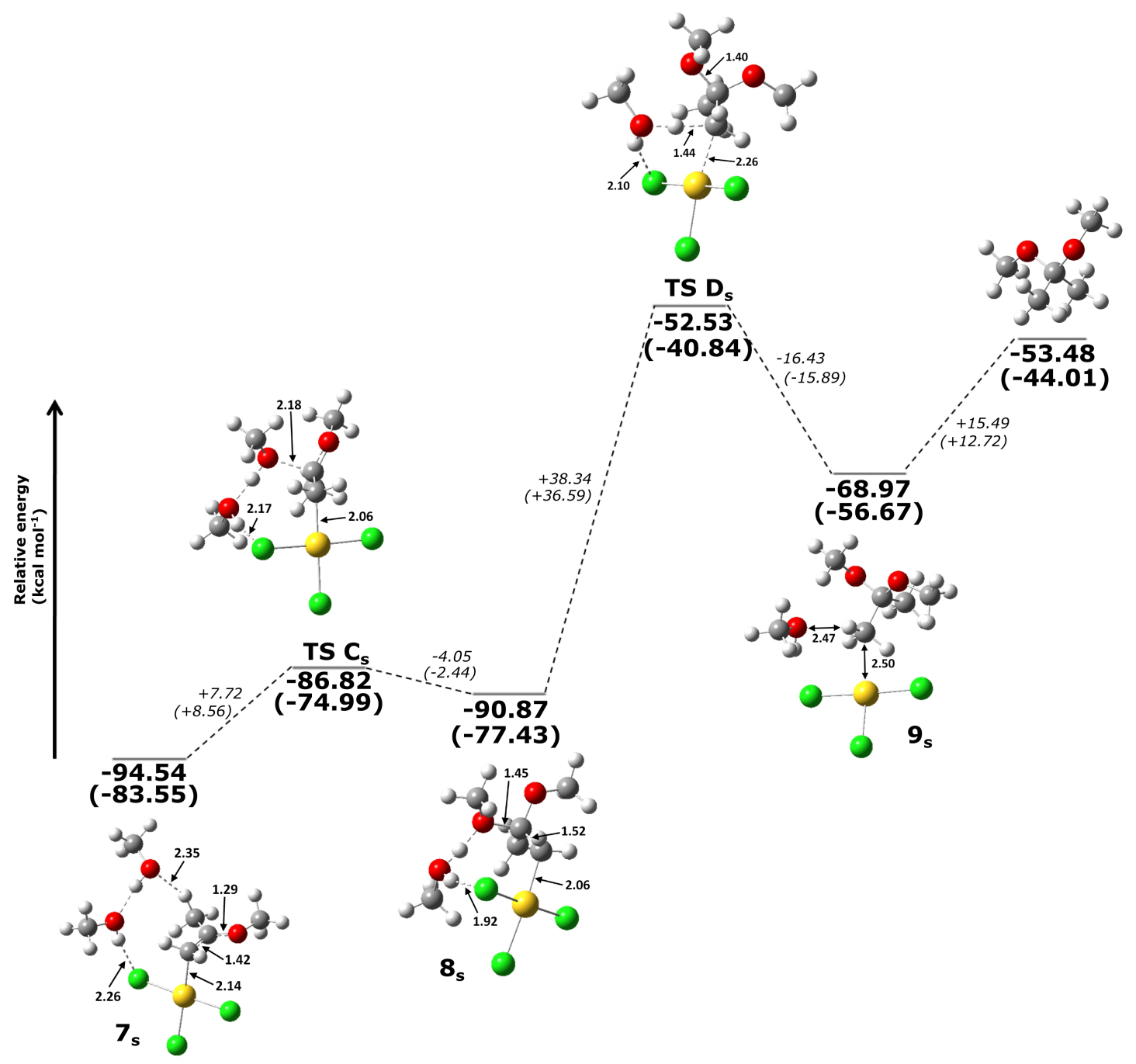

Figure 3.4 Third part of the mechanism. Relative energies with respect to the starting materials of PBE0 calculations are given below structures $\mathbf{7}_{\mathrm{S}}$ to the product respectively while energetic changes with respect to the previous structures are given on the dotted lines that represent the reaction coordinate. Values for BP86 calculations are given in parentheses. The structural parameters are in $\AA$. All energies in $\mathrm{kcal} \mathrm{mol}^{-1}$. 


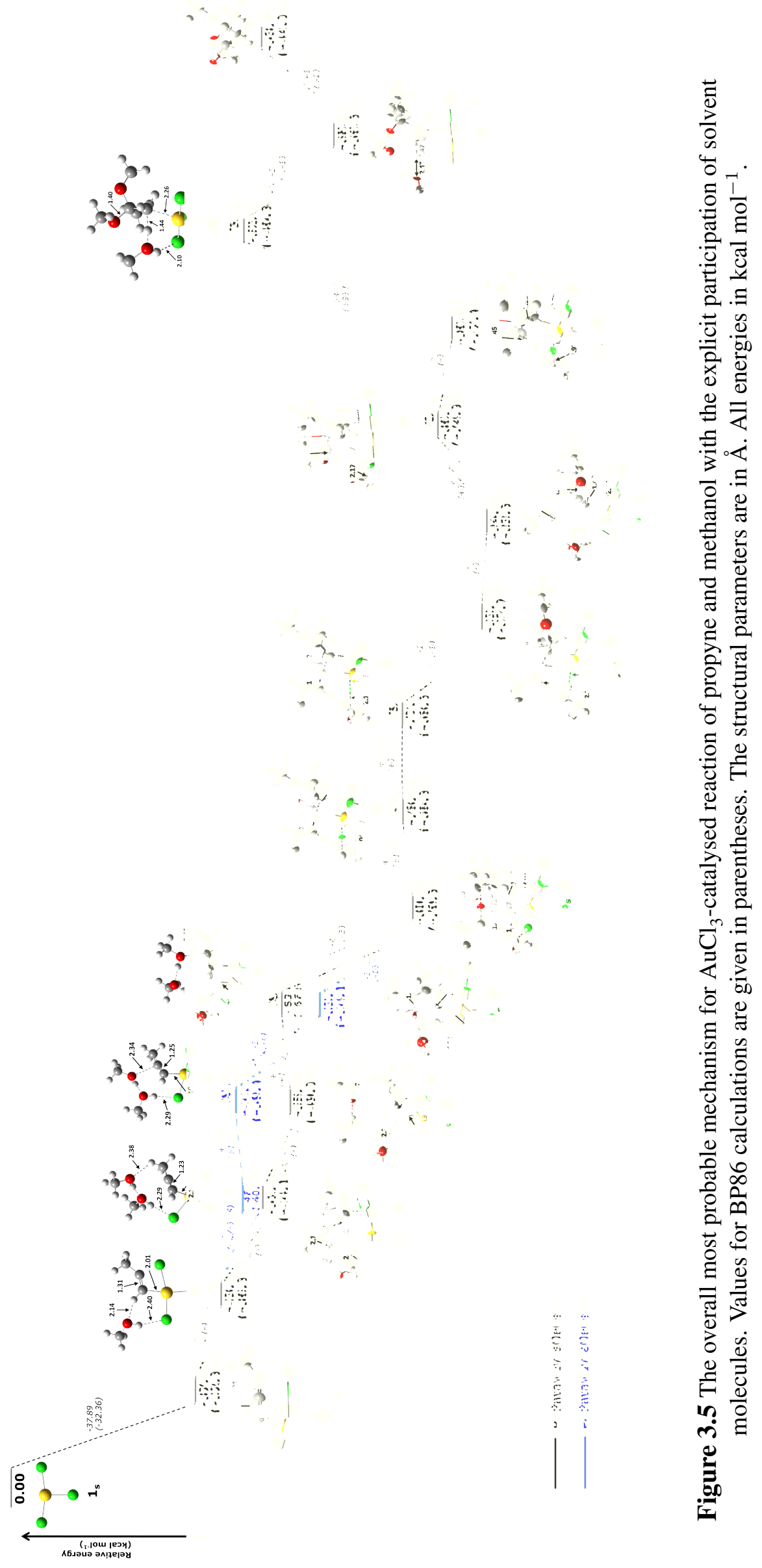




\subsubsection{Comparison between singlet and triplet states}

Next, the possibility of triplet spin state among the intermediates in the mechanism was investigated. In order to investigate whether the mechanism follows a pathway of singlet state intermediates or triplet states, we located the local minima of several key intermediates $\left(\mathbf{1}_{\mathrm{s}}, \mathbf{2}_{\mathrm{s}}, \mathbf{3}_{\mathrm{s}}\right.$ and $\left.\mathbf{7}_{\mathrm{s}}\right)$ in their triplet state. The results showed that the triplet state structures are significantly higher in energy than the singlet state structures (See Table 3.1). The results suggest that the stationary points remain as singlet states throughout the mechanism.

Table 3.1 Energy difference, $\Delta \mathrm{E}$ between triplet and singlet state structures computed at PBE0 and BP86 functional. All values are in $\mathrm{kcal} \mathrm{mol}^{-1}$.

\begin{tabular}{c|rr}
\hline \multirow{2}{*}{ Structure } & \multicolumn{2}{|c}{$\Delta \mathbf{E}$} \\
\cline { 2 - 3 } & PBE0 & BP86 \\
\hline $\mathbf{1}_{\mathrm{s}}$ & 9.8 & 6.3 \\
$\mathbf{2}_{\mathrm{s}}$ & 24.6 & 18.7 \\
$\mathbf{3}_{\mathrm{s}}$ & 25.2 & 19.8 \\
$\mathbf{7}_{\mathrm{s}}$ & 36.1 & 33.8 \\
\hline
\end{tabular}

\subsection{Comparison of $\mathrm{H}_{2} \mathrm{O}$ - and $\mathrm{CH}_{3} \mathrm{OH}$-nucleophilic addi- tion mechanisms}

The reaction mechanism was found to proceed slightly different when water is present in the reaction than for the methanol case. As mentioned previously in the Introduction (Chapter 1), the mechanism with water leads to the formation of a ketone product while the reaction with alcohol leads to ketal formation. The two mechanisms are rather straightforward, with the nucleophilic attack leading the reaction, followed by hydrogen migration. In the gold-catalysed hydration of alkynes, methanol is often used as the solvent in the presence of water in the reaction. It is of our interest to investigate how the two nucleophiles compete in the reaction. In a paper by Sicilia et al., ${ }^{116}$ it was 
reported that the nucleophilic attack by methanol would be more preferable than water which leads to the prediction that the ketone is formed from the enol ether intermediate. In contrast, Lein et al. ${ }^{117}$ predicted that the enol intermediate is formed in their solventassisted mechanism. Our findings from BP86 calculations showed that the nucleophilic attack by methanol requires about $1.2-1.4 \mathrm{kcal} \mathrm{mol}^{-1}$ (Figure 3.2) while the addition of water has an activation energy of only $0.2 \mathrm{kcal} \mathrm{mol}^{-1} .{ }^{117}$ The difference between these two predictions is the participation of explicitly included solvent molecules in the mechanism. The participation of solvent molecules decreases the barrier of both nucleophilic attacks but due to the stronger hydrogen bonding interactions among water molecules, the barrier for water addition is decreased even further than for its methanol counterparts which is the main reason for the discrepancy between the two predictions. However, it is strongly believed that the solvent-assisted mechanism provides a better representation of the real reaction as it shows the role of the solvents in assisting the reaction processes. Therefore, it can be concluded that the ketone is actually produced from a tautomerisation of an enol intermediate and not from an enol ether intermediate.

The hydrogen migration in the $\mathrm{H}_{2} \mathrm{O}$-mechanism was assisted by only one extra water molecule. However, in the $\mathrm{CH}_{3} \mathrm{OH}$-mechanism, the hydrogen migration step does not happen until two other methanol molecules come into play. The hydrogen migration for both reactions has a small activation barrier, with the $\mathrm{H}_{2} \mathrm{O}$-mechanism having a higher barrier $\left(1.3 \mathrm{kcal} \mathrm{mol}^{-1}\right)$ than the the $\mathrm{CH}_{3} \mathrm{OH}$-mechanism $\left(0.2 \mathrm{kcal} \mathrm{mol}^{-1}\right)$. Again, this step demonstrates the importance of solvent molecules' participation in the mechanism. The main difference between these two mechanisms is that deprotonation and migration of the hydrogen are concerted in the $\mathrm{H}_{2} \mathrm{O}$-mechanism. In contrast, the attacking methanol is first deprotonated by nearby solvent molecule in the $\mathrm{CH}_{3} \mathrm{OH}$-mechanism, forming a metastable structure in the preceding step which enables the hydrogen migration to occur. 


\subsection{Conclusions}

The results describe the reaction mechanism of the gold-catalysed ketal formation with an acetylene derivative as starting material. The individual steps highlight the importance of hydrogen bonding networks to facilitate the two nucleophilic attacks as well as for the migrations of the two hydrogen atoms after the addition steps. We predict that both nucleophilic attacks proceed fast over low activation barriers. Particularly the first attack appears to proceed almost without a barrier, irrespective of whether two or three solvent molecules are involved in the hydrogen bonding network that facilitates and directs the reaction. The second nucleophilic attack is calculated to be somewhat slower. The two pathways for the first hydrogen migration are able to clearly show how the solvent molecules actively facilitate the mechanism. Both the large barrier and the relative destabilisation of the product can be explained by the lack of a suitable coordination site for the remaining catalyst molecule. The exact nucleophile concentration dependence of the kinetics is unlikely to have been observed before because the reaction in question is usually carried out in a fashion where the nucleophile also serves as the solvent. However, using the calculated rate constants of the reaction, a theoretical prediction of how the concentration of intermediates changes over time can be made and this will be discussed in more details in Chapter 6.3. On that note, we would also like to encourage a thorough experimental investigation into the overall rate at which this reaction proceeds. 


\section{Chapter 4}

\section{Results and Discussions (Reaction 2):}

\section{Mechanism of the Reaction of 4,4-dimethylpent-2-yne with Methanol Catalysed by $\mathrm{AuCl}_{3}$}

To investigate the extent of which the hydrogen bonding network assists the mechanism, all the hydrogens in propyne from the first reaction (Chapter 3) are replaced by methyl groups and the reactions are given below.

$$
\begin{aligned}
& \mathrm{CH}_{3} \mathrm{C} \equiv \mathrm{CC}\left(\mathrm{CH}_{3}\right)_{3}+2 \mathrm{CH}_{3} \mathrm{OH} \stackrel{+\mathrm{AuCl}_{3}}{\longrightarrow} \mathrm{CH}_{3} \mathrm{CH}_{2} \mathrm{C}\left(\mathrm{OCH}_{3}\right)_{2} \mathrm{C}\left(\mathrm{CH}_{3}\right)_{3} \\
& \mathrm{CH}_{3} \mathrm{C} \equiv \mathrm{CC}\left(\mathrm{CH}_{3}\right)_{3}+2 \mathrm{CH}_{3} \mathrm{OH} \stackrel{+\mathrm{AuCl}_{3}}{\longrightarrow} \mathrm{CH}_{3} \mathrm{C}\left(\mathrm{OCH}_{3}\right)_{2} \mathrm{CH}_{2} \mathrm{C}\left(\mathrm{CH}_{3}\right)_{3}
\end{aligned}
$$

The bulkier substrate is 4,4-dimethylpent-2-yne and to ease the discusssion, the numbering of some carbon atoms that will be discussed is as shown in Figure 4.1.

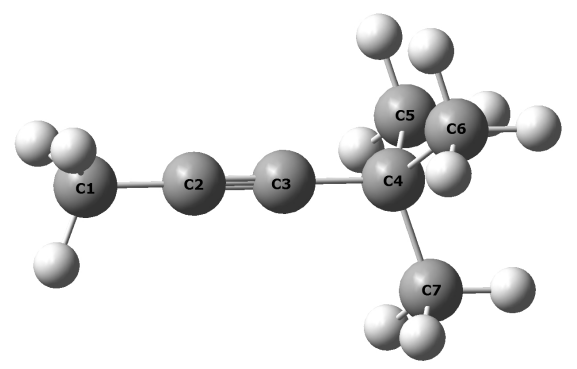

Figure 4.1 The numbering of carbon atoms in the reactant.

\subsection{Reaction sequence}

In the first step of the reaction, the $\mathrm{AuCl}_{3}$ catalyst $\mathbf{1}_{\mathrm{m}}$ coordinates with the heptyne reactant to form an activated complex. This activated complex was found to have two 
conformations of perpendicular $\left(\mathbf{2}_{\mathrm{m}}\right)$ and in-plane $\left(\mathbf{2}_{\mathrm{m}}^{\prime}\right.$ and $\left.\mathbf{2}^{\prime \prime}{ }_{\mathrm{m}}\right)$ orientation, similar to the ones found in Reaction 1 (Chapter 3). For the perpendicular orientation, it was observed that the catalyst resides symmetrically in between $\mathrm{C} 2$ and $\mathrm{C} 3$ while the in-plane conformations show a different behaviour. As for the in-plane structures, the catalyst was found to coordinate either at $\mathrm{C} 2$ or at $\mathrm{C} 3$ as shown in Figure 4.2. The perpendicular oriented activated complex was found to be $10.5 \mathrm{kcal} \mathrm{mol}^{-1}$ more stable than the inplane, $\mathbf{2}^{\prime}{ }_{\mathrm{m}}$ structure (where the catalyst is coordinated to the $\mathrm{C} 2$ atom) and $3.8 \mathrm{kcal} \mathrm{mol}^{-1}$ more stable than the in-plane, $\mathbf{2}^{\prime \prime}{ }_{\mathrm{m}}$ orientation (where the catalyst is coordinated to the $\mathrm{C} 3$ atom). This perpendicular orientation displays bonding in $\eta^{2}$ fashion with the $\pi$ system of the substrate. Following the formation of the more stable conformer $\left(\mathbf{2}_{\mathrm{m}}\right)$, the energy profile goes energetically downwards by $37.0 \mathrm{kcal} \mathrm{mol}^{-1}$ from $\mathbf{1}_{\mathrm{m}}$.
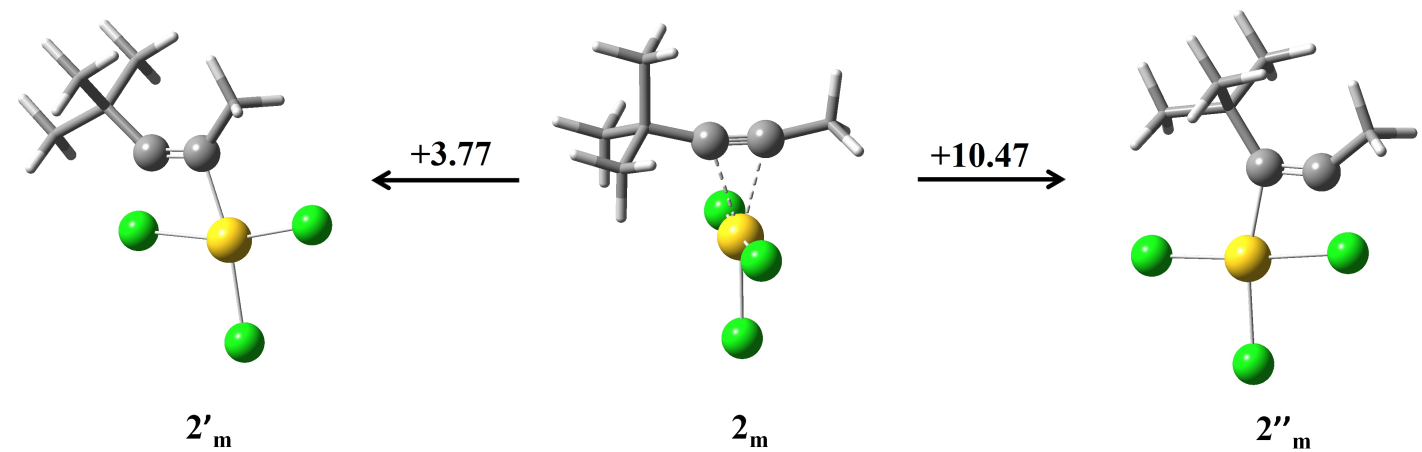

Figure 4.2 The perpendicular, $\mathbf{2}_{\mathrm{m}}$ and in-plane conformations, $\mathbf{2}_{\mathrm{m}}^{\prime}$ and $\mathbf{2}^{\prime \prime}{ }_{\mathrm{m}}$ of $\mathrm{AuCl}_{3}$-heptyne activated complex. The energetic changes from PBE0 calculations are given in $\mathrm{kcal} \mathrm{mol}^{-1}$.

The first methanol then enters the mechanism in the following step, establishing an interaction with the activated complex $\mathbf{2}_{\mathrm{m}}$ to form $\mathbf{3}_{\mathrm{m}}$. The interaction formed further stabilises the system by $6.1 \mathrm{kcal} \mathrm{mol}^{-1}$. It becomes obvious that another methanol is needed for the reaction to occur since the first methanol is not positioned for nucleophilic attack in $\mathbf{3}_{\mathrm{m}}$. Besides that, it has been stressed before that participation of an extra solvent molecule is needed to lower the activation barrier of the nucleophilic attack. The second nucleophile comes into scene to form $\mathbf{4}_{\mathrm{m}}$ which has an extended hydrogen bonding network between the two oxygen-nucleophiles. The addition of the second methanol stabilises the system by $8.3 \mathrm{kcal} \mathrm{mol}^{-1}$. The extended network allows the 
second methanol to be positioned well for a nucleophilic attack. However, since the substrate is an internal alkyne, it suffers from a regioselectivity problem due to the two possible reaction sites at $\mathrm{C} 2$ and $\mathrm{C} 3$. This causes the reaction to split into two mechanisms that lead to different products.

\subsubsection{Mechanism 1, M1}

In this section, the mechanism in which the nucleophilic attacks occur at the $\mathrm{C} 3$ atom will be discussed and denoted as Mechanism 1, M1. This mechanism represents one of the possible reaction pathways in the hydroalkoxylation of internal heptyne and the feasibility of this pathway will be elaborated further at the end of the chapter.

\section{M1-Pathway 2MeOH-m}

The second methanol is in position for nucleophilic attack at the $\mathrm{C} 3$ atom in $\mathbf{4}_{\mathrm{m}}$ and this represents the beginning of Mechanism 1, M1. The attack proceeds with an activation

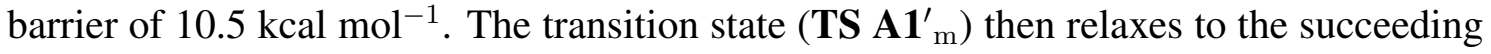
minimum $\mathbf{5 - 1}{ }_{\mathrm{m}}^{\prime}$ energetically downwards by $10.7 \mathrm{kcal} \mathrm{mol}^{-1}$. As the reaction proceeds, the distance between the nucleophilic-O and $\mathrm{C} 3$ decreases from $2.07 \AA$ in $\mathbf{T S ~} \mathbf{A} \mathbf{1}_{\mathrm{m}}^{\prime}$ to $1.43 \AA$ in the product. Following the formation of $\mathbf{5 - 1}_{\mathrm{m}}^{\prime}$, the hydrogen migrates from the coordinated methanol to the $\mathrm{C} 2$ atom with an activation energy of only $0.2 \mathrm{kcal} \mathrm{mol}^{-1}$. The extra solvent methanol forms a hydrogen bond with the migrating hydrogen and guides the atom into close proximity to the possible reaction site $\left(1.48 \AA\right.$ in $\left.\mathbf{T S ~ B 1}{ }_{\mathrm{m}}^{\prime}\right)$. The

hydrogen then coordinates to $\mathrm{C} 2$ at $1.09 \AA$ and forms the product $\mathbf{6 - \mathbf { 1 } ^ { \prime }}{ }_{\mathrm{m}}$, completing the first nucleophilic attack in this pathway. This pathway is highlighted in blue in Figure 4.3. 


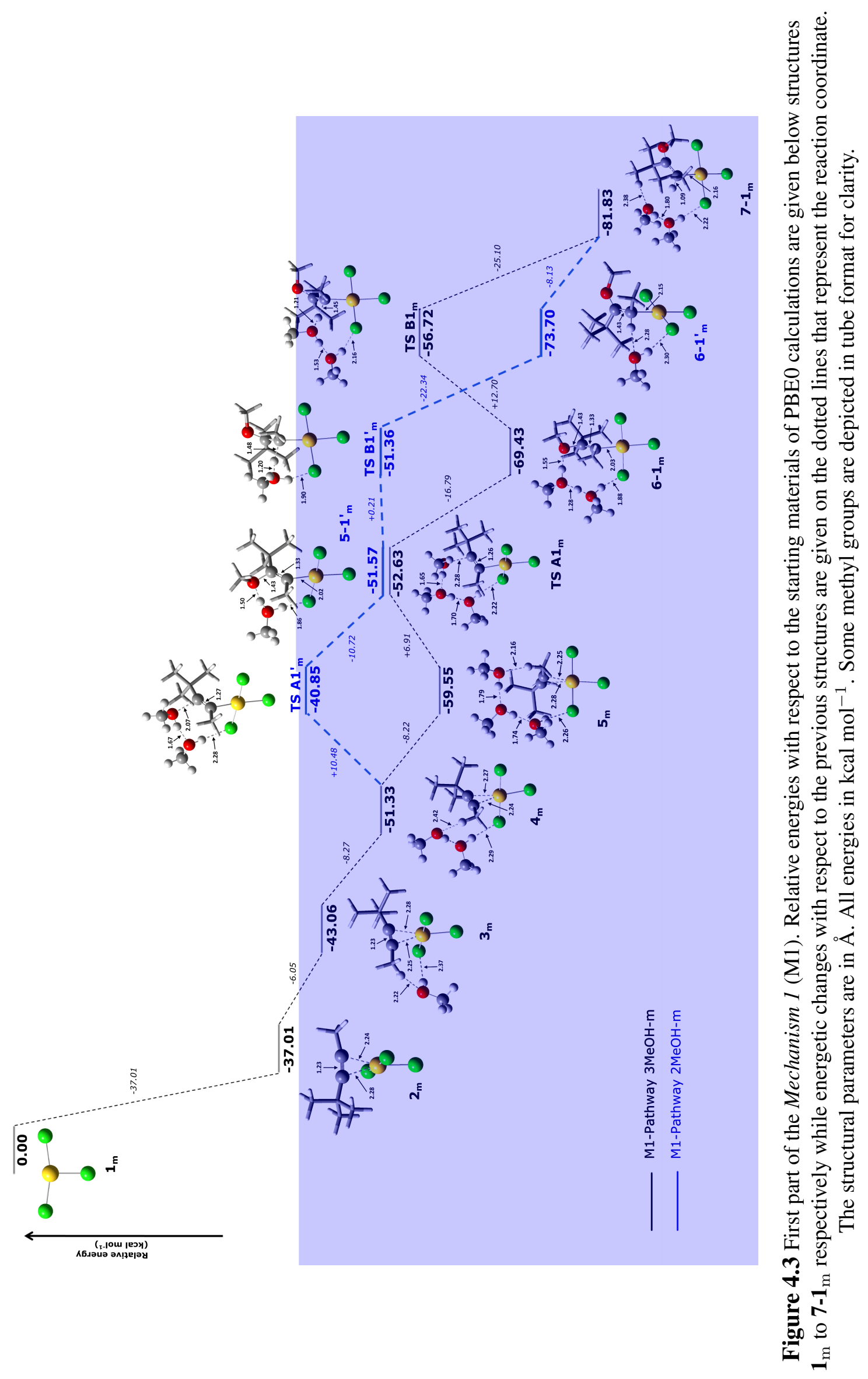




\section{M1-Pathway 3MeOH-m}

Similar to the Reaction 1 (Chapter 3), the formation of $\mathbf{4}_{\mathrm{m}}$ can also lead to an alternative pathway (Figure 4.3) where the nucleophile does not attack the substrate until the third methanol is added into the mechanism, forming $\mathbf{5}_{\mathrm{m}}$. With the third methanol in position, the nucleophilic attack proceeds with an activation energy of $6.9 \mathrm{kcal} \mathrm{mol}^{-1}\left(\mathbf{T S ~ A} \mathbf{1}_{\mathrm{m}}\right)$. This nucleophilic attack resembles the one in M1-Pathway $2 \mathrm{MeOH}-\mathrm{m}$ but with an extra participating solvent molecule. The energy profile then goes energetically downwards by $16.8 \mathrm{kcal} \mathrm{mol}^{-1}$ from $\mathbf{T S ~} \mathbf{A} \mathbf{1}_{\mathrm{m}}$ to form $\mathbf{6}-\mathbf{1}_{\mathrm{m}}$. The mechanism is followed by a hydrogen migration after the nucleophilic attack. With the presence of explicit solvent molecules in the mechanism, the hydrogen migrates from the coordinated methanol to the $\mathrm{C} 2$ atom. From the structure of $\mathbf{6 - 1} \mathbf{1}_{\mathrm{m}}$, it was seen that the solvent molecules have two roles in assisting the migration. One of them acts as the deprotonating agent that actively transfers the hydrogen to $\mathrm{C} 2$ while the other solvent molecule acts as an 'anchor' through hydrogen bonding with the chloro ligand in the catalyst $(\mathrm{H} \cdots \mathrm{Cl})$. This 'anchor' holds the other solvent molecule in place for a successful migration. The hydrogen migration involves overcoming a barrier of $12.7 \mathrm{kcal} \mathrm{mol}^{-1}\left(\mathbf{T S ~ B 1} \mathbf{m}_{\mathrm{m}}\right)$. The system then goes energetically downwards by $25.1 \mathrm{kcal} \mathrm{mol}^{-1}$, forming the end product of the first nucleophilic attack, $7-1_{m}$.

\section{Second nucleophilic attack of M1}

In M1-Pathway 2MeOH-m, there was only one methanol present in the mechanism that acted as the nucleophile for the second addition. Based on our preliminary results, the mechanism is always accompanied by at least one additional methanol that acts as a solvent to assist the nucleophilic attack. Hence, the third methanol is added to $\mathbf{6 - 1}{ }_{\mathrm{m}}$ that results in the formation of $\mathbf{7 - \mathbf { 1 } _ { \mathrm { m } }}$. This also leads to the convergence of the two

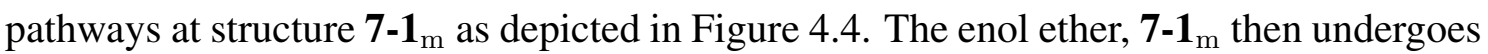


another nucleophilic attack by methanol in analogy to the first nucleophilic addition. The activation energy for this step is significantly higher, with $\mathbf{T S} \mathbf{C} \mathbf{1}_{\mathrm{m}}$ sitting on a $24.1 \mathrm{kcal} \mathrm{mol}^{-1}$ energy barrier with respect to its preceding minimum. The minimum that follows $\left(\mathbf{8 - 1} \mathbf{1}_{\mathrm{m}}\right)$ is only $0.2 \mathrm{kcal} \mathrm{mol}^{-1}$ lower in energy with respect to $\mathbf{T S} \mathbf{C} \mathbf{1}_{\mathrm{m}}$ and the similarity in the structure of $\mathbf{8}-\mathbf{1}_{\mathrm{m}}$ and $\mathbf{T S} \mathbf{C} \mathbf{1}_{\mathrm{m}}$ indicates that the transition state is a late one for this step. A point worthy of note is that this step is endothermic by $23.8 \mathrm{kcal} \mathrm{mol}^{-1}$. The nucleophile was found to be approximately $1.66 \AA$ away from the reaction site and this distance decreases to $1.59 \AA$ in $\mathbf{8 - 1}$. As can be seen in the structure of $\mathbf{8 - 1} \mathbf{1}_{\mathrm{m}}$, the solvent molecule is already in place to deprotonate the attacking methanol nucleophile. The hydrogen migration immediately follows after the nucleophilic attack

with its transition state TS D1 $\mathbf{m}_{\mathrm{m}}$ sitting on a $35.4 \mathrm{kcal} \mathrm{mol}^{-1}$ barrier. The energy curve then goes downwards by $27.3 \mathrm{kcal} \mathrm{mol}^{-1}$ to form the final product of the reaction. Similar to the first reaction discussed in Chapter 3, the rate determining step is expected to be the last hydrogen migration as it requires the largest activation energy. With the $\mathrm{C} 2$ atom being saturated after the hydrogen migration, the catalyst has no place to coordinate in the substrate leading to dissociation from the product and a large activation barrier. The final ketal product can already be seen in structure $\mathbf{9 - 1} \mathbf{m}_{\mathrm{m}}$ and with the elimination of the gold catalyst and excess solvent molecule, the cycle restarts with another heptyne substrate in the reaction.

\subsubsection{Mechanism 2, M2}

In this section, the mechanism in which the nucleophilic attacks occur at the $\mathrm{C} 2$ atom will be discussed and denoted as Mechanism 2, M2. This mechanism represents an alternative reaction pathway for the hydroalkoxylation of the internal alkyne under study. The feasibility of this mechanism will be compared to M1 and discussed in more details at the end of this chapter. 


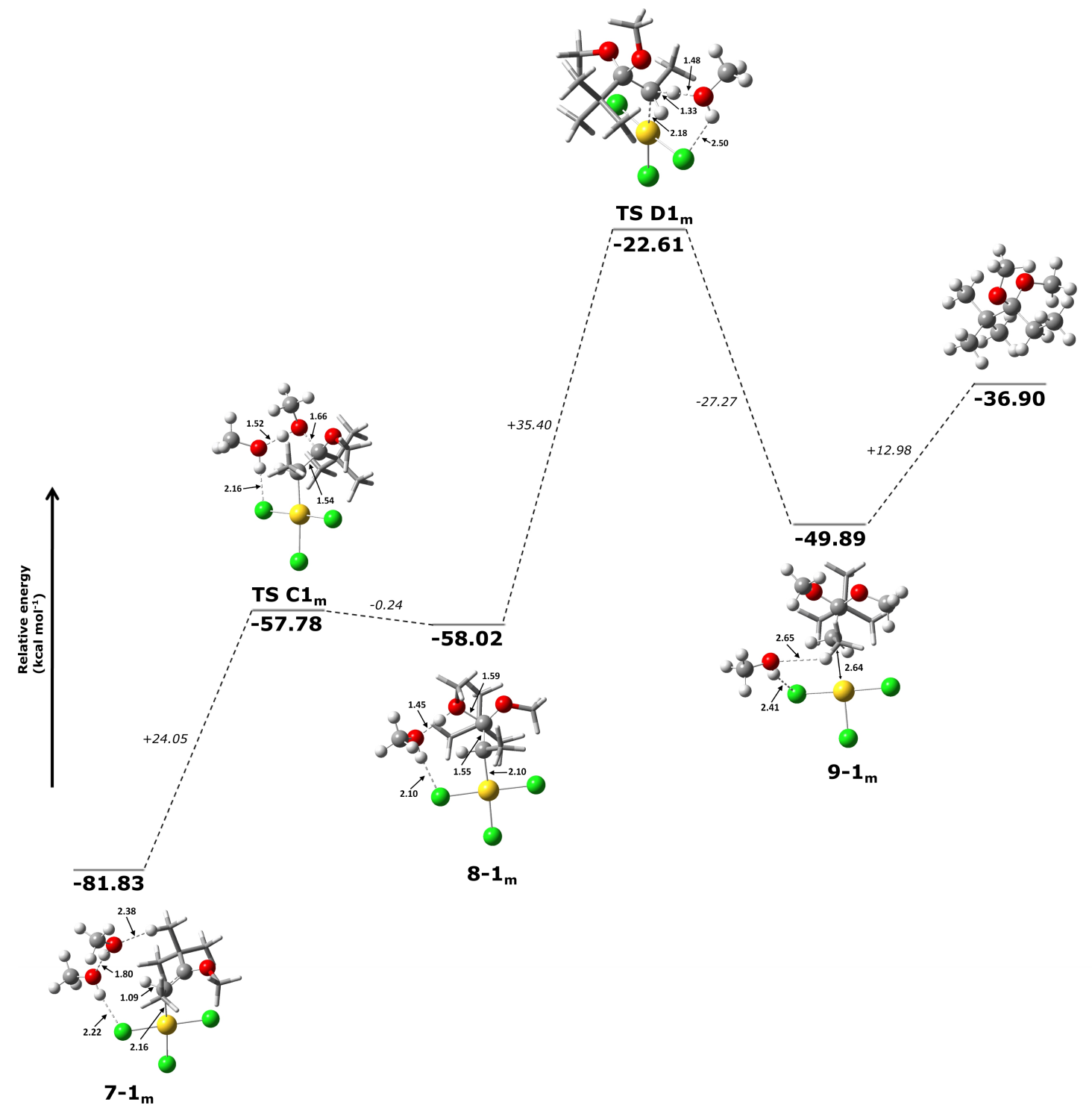

Figure 4.4 Second part of the Mechanism 1 (M1). Relative energies with respect to the starting materials of PBE0 calculations are given below structures $\mathbf{7 - 1} \mathrm{m}$ to the product respectively while energetic changes with respect to the previous structures are given on the dotted lines that represent the reaction coordinate. The structural parameters are in $\AA$. All energies in $\mathrm{kcal} \mathrm{mol}^{-1}$. Some methyl groups are depicted in tube format for clarity.

\section{M2-Pathway 2MeOH-m}

The oxygen-nucleophile can also choose to attack $\mathrm{C} 2$ atom in $\mathbf{4}_{\mathrm{m}}$ and this leads to Mechanism 2, M2. The pathway in M2 is similar to M1-Pathway 2MeOH-m. The nucleophile attacks the substrate with an activation barrier of $10.7 \mathrm{kcal} \mathrm{mol}^{-1}$. The energy profile then goes energetically downwards by $11.5 \mathrm{kcal} \mathrm{mol}^{-1}$ to form $\mathbf{5 - 2}_{\mathrm{m}}{ }_{\mathrm{m}}$. The 
distance between the nucleophilic-O and C2 decreases from $1.99 \AA$ in $\mathbf{T S} \mathbf{A 2}^{\prime}{ }_{\mathrm{m}}$ to $1.43 \AA$ in the $\mathbf{5 - 2}{ }_{\mathrm{m}}^{\prime}$ as the reaction proceeds. The hydrogen migration follows immediately after the nucleophilic attack with a barrier of $1.6 \mathrm{kcal} \mathrm{mol}^{-1}$. The migration occurs with the participation of the additional solvent methanol that brings the migrating hydrogen close to the reaction site $(1.57 \AA)$. The step is complete with the formation of the product of the first nucleophilic attack, $\mathbf{6 - 2}{ }_{\mathrm{m}}^{\prime}$ and the newly formed $\mathrm{C}-\mathrm{H}$ bond length was found to be $1.09 \AA$. This pathway is highlighted in blue in Figure 4.5. 


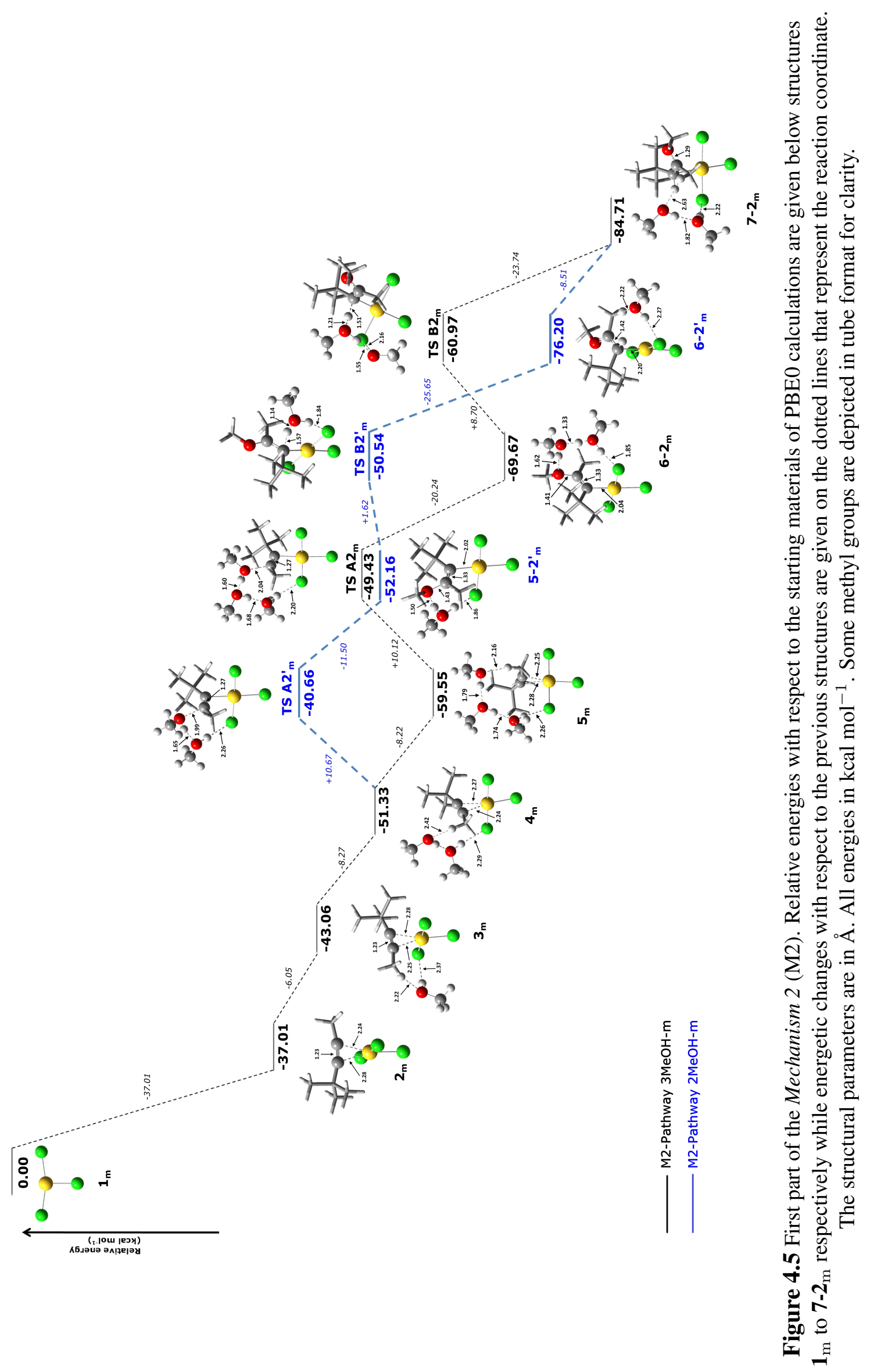




\section{M2-Pathway 3MeOH-m}

The mechanism can also take an alternative pathway which has two assisting solvent molecules. With the inclusion of the third methanol molecule, the mechanism diverges into M2-Pathway $3 \mathrm{MeOH}-\mathrm{m}$ as shown in Figure 4.5. The third methanol is in position for the nucleophilic attack and the step requires an activation energy of $10.1 \mathrm{kcal} \mathrm{mol}^{-1}$ with the nucleophilic-O at $2.04 \AA$ away from $\mathrm{C} 2$ atom as depicted in $\mathbf{T S} \mathbf{A} \mathbf{2}_{\mathrm{m}}$. The product, 6-2 $2_{\mathrm{m}}$ that formed is $20.2 \mathrm{kcal} \mathrm{mol}^{-1}$ lower in energy than $\mathbf{T S} \mathbf{A} \boldsymbol{2}_{\mathrm{m}}$. The mechanism is followed by hydrogen migration, assisted by the participating solvent methanols from the coordinated methanol to the $\mathrm{C} 3$ atom. The two roles of the solvent molecules seen in M1 is also apparent in M2. Similarly, one of the assisting solvent methanol (anchor) holds the other solvent molecule in position to deprotonate the hydrogen from the enol ether oxygen atom. In a concerted process, the solvent molecules direct the hydrogen to the $\mathrm{C} 3$ atom to complete the migration. The transition state for this step (TS B2 $2_{\mathrm{m}}$ ) sits on top of a $8.7 \mathrm{kcal} \mathrm{mol}^{-1}$ potential energy hill. The system then goes energetically downwards by $23.7 \mathrm{kcal} \mathrm{mol}^{-1}$, forming the end product of the first nucleophilic attack, $\mathbf{7 - 2} \mathrm{m}$.

\section{Second nucleophilic attack of M2}

For the second nucleophilic attack to proceed from M2-Pathway $2 \mathrm{MeOH}-\mathrm{m}$, an additional

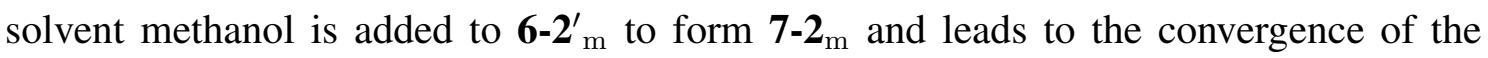
two pathways at this point as depicted in Figure 4.6. The enol ether, $\mathbf{7 - 2} \mathrm{m}$ undergoes a structural rearrangement to form intermediate $\mathbf{7 - 2} \mathbf{a}_{\mathrm{m}}$ before the nucleophilic attack occurs. This intermediate is $0.9 \mathrm{kcal} \mathrm{mol}^{-1}$ higher in energy and it was found that the hydrogen bonding network is formed with the axial chloro ligand to hold the nucleophile in place for the next step. With the methanol being in close proximity to the $\mathrm{C} 2$ atom, the nucleophilic attack occurs with an activation barrier of $9.9 \mathrm{kcal} \mathrm{mol}^{-1}$. The process is represented by transition state $\mathbf{T S} \mathbf{C} \mathbf{2}_{\mathrm{m}}$ whereby the attacking nucleophile is about 
$1.96 \AA$ from the reaction site. The mechanism then goes energetically downwards by $3.2 \mathrm{kcal} \mathrm{mol}^{-1}$, producing intermediate $\mathbf{8 - 2} \mathrm{m}$. A point worthy of note is that this step is endothermic in nature, with $\mathbf{8 - 2}$ being $6.7 \mathrm{kcal} \mathrm{mol}^{-1}$ higher in energy than $\mathbf{7 - 2} \mathrm{m}$. Structure $\mathbf{8 - 2}$ m represents the starting point of the second hydrogen migration. The solvent molecule is in place to deprotonate the coordinated methanol and initiates the migration process. The hydrogen migration requires a very high activation barrier of $53.3 \mathrm{kcal} \mathrm{mol}^{-1}$, as represented by its transition state TS $\mathbf{D 2}{ }_{\mathrm{m}}$, whereby the hydrogen is $1.53 \AA$ away from the reaction site. The energy profile then goes energetically downwards by $34.3 \mathrm{kcal} \mathrm{mol}^{-1}$ to form the final product of the reaction. Similar to Reaction 1 discussed in Chapter 3, the rate determining step based on the calculation is the last hydrogen migration as it requires the largest activation energy. With the $\mathrm{C} 3$ atom being saturated after the hydrogen migration, the catalyst has no place to coordinate in the substrate leading to dissociation from the product and hence the high activation energy. The final ketal product can already be seen in structure $\mathbf{9 - 2}$. Following the elimination of the gold catalyst and excess solvent molecule, the product is formed and the catalytic cycle repeats itself with the gold catalyst coordinating to another heptyne substrate. 


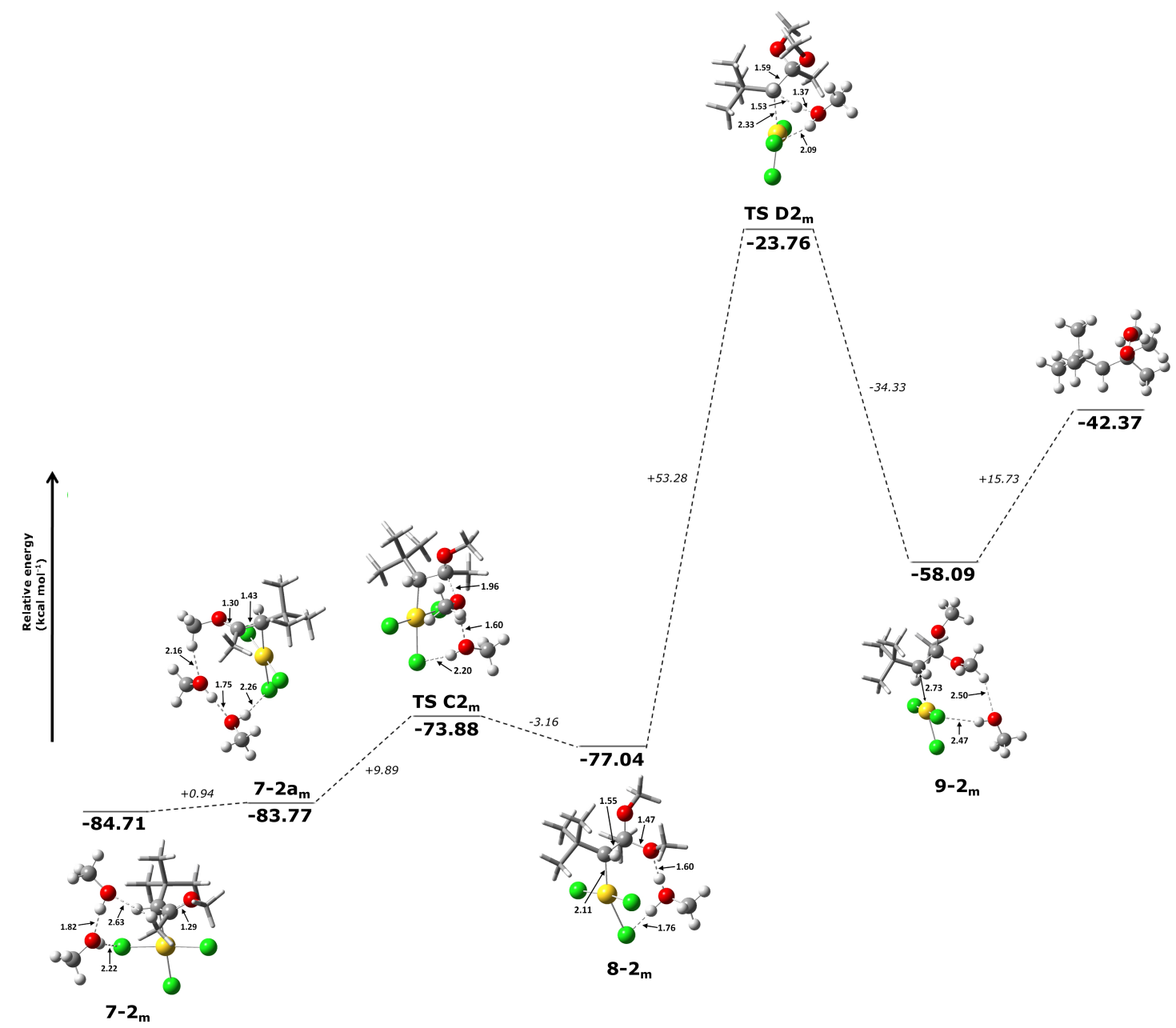

Figure 4.6 Second part of the Mechanism 2 (M2). Relative energies with respect to the starting materials of PBE0 calculations are given below structures $\mathbf{7 - 2}$ m to the product respectively while energetic changes with respect to the previous structures are given on the dotted lines that represent the reaction coordinate. The structural parameters are in $\AA$. All energies in $\mathrm{kcal} \mathrm{mol}^{-1}$. Some methyl groups are depicted in tube format for clarity.

\subsubsection{Comparison between singlet and triplet states}

The feasibility of the reactions following a triplet state pathway was investigated. In order to determine whether the mechanisms follow a pathway of singlet state intermediates or triplet states, we located the local minima of several key intermediates $\left(\mathbf{1}_{\mathrm{m}}, \mathbf{2}_{\mathrm{m}}, \mathbf{3}_{\mathrm{m}}, \mathbf{7 - \mathbf { 1 } _ { \mathrm { m } }}\right.$ and $\mathbf{7 - 2} \mathrm{m}$ ) in their triplet state. The results showed that the triplet state structures are significantly higher in energy than the singlet state structures (See Table 4.1). The results further substantiate our assumption that the reaction follows the singlet state mechanism. 
Table 4.1 Energy difference, $\Delta \mathrm{E}$ between triplet and singlet state structures computed at PBE0 functional. All values are in $\mathrm{kcal} \mathrm{mol}^{-1}$.

\begin{tabular}{c|c}
\hline Structure & $\Delta \mathbf{E}$ \\
\hline $\mathbf{1}_{\mathrm{m}}$ & 9.81 \\
$\mathbf{2}_{\mathrm{m}}$ & 39.31 \\
$\mathbf{3}_{\mathrm{m}}$ & 38.74 \\
$\mathbf{7 - 1}_{\mathrm{m}}$ & 38.27 \\
$\mathbf{7 - 2}_{\mathrm{m}}$ & 38.02 \\
\hline
\end{tabular}

\subsection{Comparison of the two mechanisms, M1 and M2}

Both mechanisms are very similar and differ only at which position the nucleophiles are added to. The point at which the reaction diverges into two mechanisms is when the first nucleophilic addition takes place. It was observed that the activation barrier for the first nucleophilic attack in M1 is lower than that in M2. The addition is more favourable by $3.2 \mathrm{kcal} \mathrm{mol}^{-1}$ in Pathway $3 \mathrm{MeOH}-\mathrm{m}$. The reason behind this can be found in the NBO analysis of the bonding orbital between the electrophilic $\mathrm{C}$ atom and nucleophilic $\mathrm{O}$ atom in $\mathbf{T S ~} \mathbf{A} \mathbf{1}_{\mathrm{m}}$ and $\mathbf{T S} \mathbf{A} \boldsymbol{2}_{\mathrm{m}}$. In $\mathbf{T S} \mathbf{A} \mathbf{1}_{\mathrm{m}}$, the energetic difference between the individual atomic orbitals that form the molecular bonding orbital is lower compared to that in TS $\mathbf{A} \boldsymbol{2}_{\mathrm{m}}$, which is $197.5 \mathrm{kcal} \mathrm{mol}^{-1}$ and $234.3 \mathrm{kcal} \mathrm{mol}^{-1}$ respectively. This means that the atomic orbitals that make up the $\mathrm{C}-\mathrm{O}$ molecular bonding orbital in $\mathbf{T S ~} \mathbf{A} \mathbf{1}_{\mathrm{m}}$ have a good energetic match and better interaction between the atomic orbitals.

As for the second nucleophilic addition, M1 suffers a significantly higher activation barrier than in M2. This is due to the steric hindrance introduced by the methoxy group following the first nucleophilic addition and the adjacent bulky tertiary butyl group, making it difficult for the second methanol to approach the reaction site (C3 atom) in M1. The difference between the activation barriers of the first and second nucleophilic attack is also more apparent in M1, which is $17.1 \mathrm{kcal} \mathrm{mol}^{-1}$ but almost insignificant in M2 $\left(0.2 \mathrm{kcal} \mathrm{mol}^{-1}\right)$. From the results, it can be concluded that the feasibility of the first nucleophilic attack is mainly governed by electronic effects while steric effects become 
the dominant factor in the second nucleophilic attack.

Judging from the activation barrier of the rate limiting step, one would expect the reaction to follow M1 as it has a lower activation barrier. However, the ketal product formed in M2 is more stable by $5.5 \mathrm{kcal} \mathrm{mol}^{-1}$ than the product formed in M1. The two methoxy groups in the product of M1 are attached to the carbon atom adjacent to the bulky tertiary butyl group which leads to a larger steric hindrance in the structure. Therefore, in comparison, we predict that M1 is kinetically more favourable while M2 is thermodynamically more favourable. At high reaction temperatures where the reaction is supplied with more energy, the reaction would most likely follow M2 to produce the corresponding ketal product, 2,2-dimethoxy-4,4-dimetylpentane. On the other hand, under low reaction temperature where the molecules do not possess enough energy to overcome the barrier of M2, the reaction is predicted to follow M1 and produce the ketal, 3,3-dimethoxy-4,4dimethylpentane. The overall mechanisms of M1 and M2 are depicted in Figure 4.7 and Figure 4.8 respectively. 


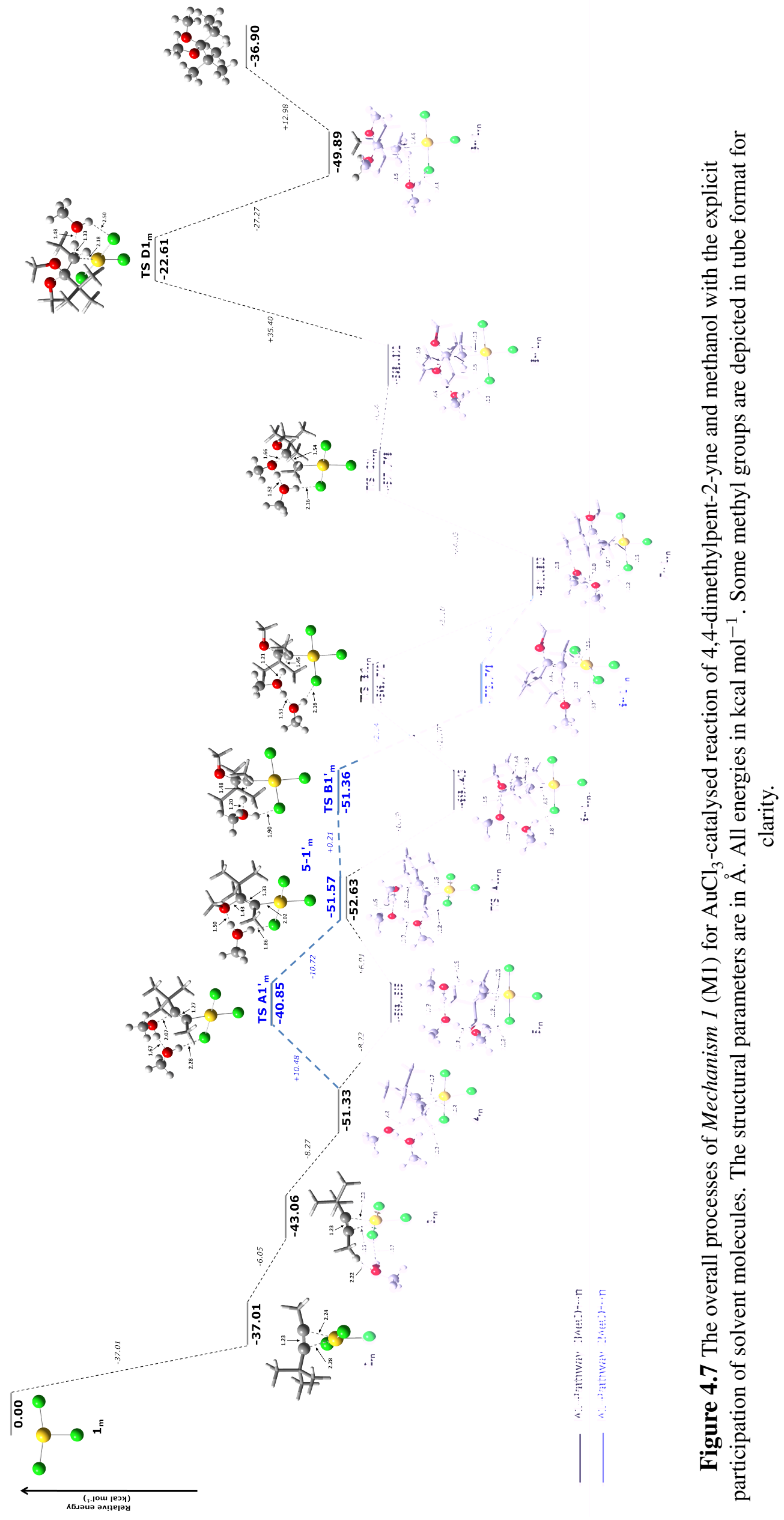




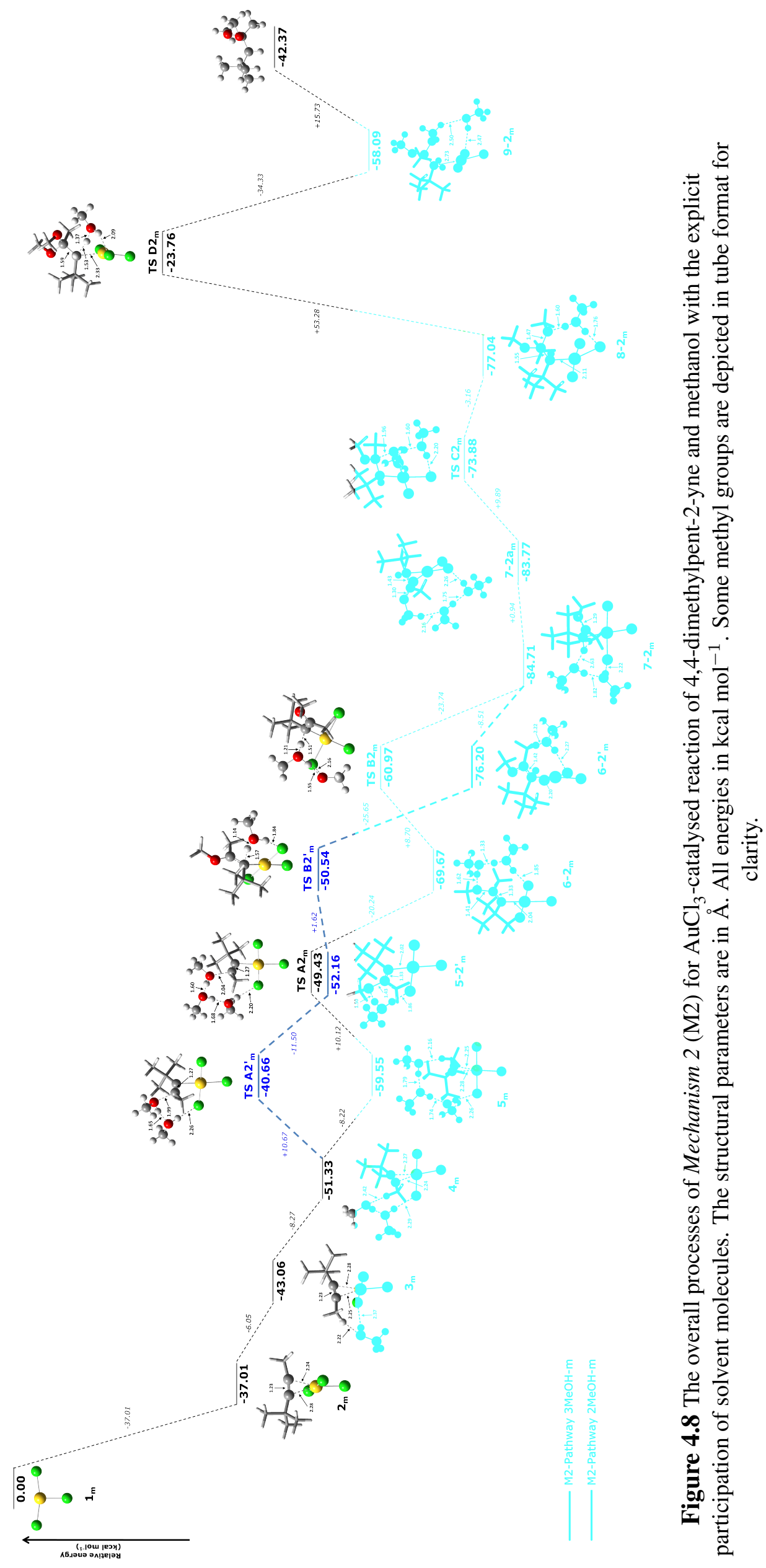




\subsection{Conclusions}

The results describe the reaction mechanism of the gold-catalysed ketal formation with an internal alkyne as the starting material. The individual steps demonstrate again the importance of hydrogen bonding networks to facilitate nucleophilic attacks and hydrogen migrations even though the bulky groups in the substrate limit the formation of hydrogen bonds with the substrate. We predict that the reaction proceeds to form a mixture of products, depending on which carbon atom the nucleophiles are added to. One of the mechanisms found in this reaction is kinetically more favourable while the other is thermodynamically more favourable, indicating that the reaction can follow either way depending on the reaction conditions. If we look at the mechanisms individually, they are very similar. The first nucleophilic attack appears to proceed at a higher rate than the second nucleophilic attack and further investigation showed that the first attack is dominated by electronic effects while the second nucleophilic attack is dominated by steric effects. Both mechanisms showed that the last hydrogen migration step is predicted to be the rate determining step for the reaction. This can be explained by the lack of a coordination site for the catalyst molecule in the substrate which leads to the catalyst dissociating from the substrate and a larger activation barrier. The two mechanisms demonstrate the complications of reactions involving internal alkynes which stem from the regioselectivity issue associated with the substrate. The main problem for such reactions is the lack of control in the mechanism due to the number of individual processes that have similar activation energies, which could result in a mixture of products that are difficult to isolate. 


\section{Chapter 5}

\section{Results and Discussions (Reaction 3):}

\section{Mechanism of the Reaction of Phenylacetylene with Methanol Catalysed by $\mathrm{Au}\left(\mathrm{C}_{6} \mathbf{F}_{5}\right) \mathrm{Cl}_{2}$}

The reaction discussed in this chapter follows the reaction that has been done experimentally by Laguna and coworkers ${ }^{25}$ and the reaction is as follows.

$$
\mathrm{HC} \equiv \mathrm{C}\left(\mathrm{C}_{6} \mathrm{H}_{5}\right)+2 \mathrm{CH}_{3} \mathrm{OH} \stackrel{+\mathrm{Au}\left(\mathrm{C}_{6} \mathrm{~F}_{5}\right) \mathrm{Cl}_{2}}{\longrightarrow} \mathrm{CH}_{3} \mathrm{C}\left(\mathrm{OCH}_{3}\right)_{2}\left(\mathrm{C}_{6} \mathrm{H}_{5}\right)
$$

The goal of our calculations was to give some insights on the reaction and compare with the experimental results. The stationary points except transition states in this mechanism were located using PBE0/aug-cc-pVTZ level of theory. Due to the large size of the system in this reaction, the calculations to locate transition states in this reaction become highly demanding and not feasible with our computer resources. To alleviate this problem, the transition states are instead located using the corresponding double- $\zeta$ basis set while their energies are calculated in the triple- $\zeta$ quality (PBE0/aug-cc-pVTZ//PBE0/aug-cc-pVDZ). However, the use of two different methods in the same study is computationally inconsistent. In order to justify the use of this approach, the energies of the local minima calculated using the two methods were compared. The values are tabulated in Table 5.1. It can be seen that the difference in energy between the two methods is within chemical accuracy of $1 \mathrm{kcal} \mathrm{mol}^{-1}$ for all the local minima in the mechanism. This indicates that the methods are comparable and it is not expected that the use of the two methods in the same study does cause a big deviation to the energetical landscape of the mechanism. Another point worthy of note is that the system's geometries optimised at the double- $\zeta$ basis set are closely similar to those calculated in the triple- $\zeta$ quality, indicating that the 
structural determination at double- $\zeta$ quality is comparable to its triple- $\zeta$ counterpart.

Table 5.1 The energetical difference $\left(\mathrm{kcal} \mathrm{mol}^{-1}\right)$ between the two methods (PBE0/aug-cc-pVTZ and PBE0/aug-cc-pVTZ//PBE0/aug-cc-pVDZ) used in the calculations.

\begin{tabular}{cccc}
\hline $\begin{array}{c}\text { Local } \\
\text { minima }\end{array}$ & $\Delta \mathbf{E}$ & $\begin{array}{c}\text { Local } \\
\text { minima }\end{array}$ & $\Delta \mathbf{E}$ \\
\hline $\mathbf{2}_{\mathrm{L}}$ & 0.87 & $\mathbf{6}_{\mathrm{L}}$ & 0.88 \\
$\mathbf{3}_{\mathrm{L}}$ & 0.90 & $\mathbf{6 a}_{\mathrm{L}}$ & 0.96 \\
$\mathbf{4}_{\mathrm{L}}$ & 0.98 & $\mathbf{7}_{\mathrm{L}}$ & 1.00 \\
$\mathbf{4}_{\mathrm{L}}^{\prime}$ & 1.02 & $\mathbf{7 a}_{\mathrm{L}}$ & 0.91 \\
$\mathbf{5}_{\mathrm{L}}^{\prime}$ & 0.84 & $\mathbf{8}_{\mathrm{L}}$ & 1.03 \\
$\mathbf{5}_{\mathrm{L}}$ & 1.03 & $\mathbf{9}_{\mathrm{L}}$ & 0.93 \\
\hline
\end{tabular}

\subsection{Reaction sequence}

The reaction starts with $\mathrm{Au}\left(\mathrm{C}_{6} \mathrm{~F}_{5}\right) \mathrm{Cl}_{2}$ catalyst $\left(\mathbf{1}_{\mathrm{L}}\right)$ coordinating to the phenylacetylene substrate to form an activated complex. The two conformations; perpendicular, $\mathbf{2}_{\mathrm{L}}$ and in-plane, $\mathbf{2}_{\mathrm{L}}^{\prime}$ orientation of the activated complex were investigated. As seen in Figure 5.1, the structure of $2_{\mathrm{L}}$ shows that the equatorial $\mathrm{Cl}-\mathrm{Au}-\mathrm{Cl}$ bonds of the catalyst are perpendicular to $\mathrm{C}-\mathrm{C}-\mathrm{C}$ bonds in the substrate while both these bonds are parallel in $\mathbf{2}_{\mathrm{L}}^{\prime}$. Structure $\mathbf{2}_{\mathrm{L}}$ is $9.9 \mathrm{kcal} \mathrm{mol}^{-1}$ more stable than $\mathbf{2}_{\mathrm{L}}^{\prime}$. Therefore, the activated complex is most likely to be formed in the perpendicular orientation and the system is stabilised by $22.1 \mathrm{kcal} \mathrm{mol}^{-1}$ with the formation of $\mathbf{2}_{\mathrm{L}}$. In analogy to the previous results, methanol enters the mechanism and forms two hydrogen bonds with the activated complex $\mathbf{2}_{\mathrm{L}}$ to form $\mathbf{3}_{\mathrm{L}}$. In $\mathbf{3}_{\mathrm{L}}$, the methanol oxygen atom coordinates to the terminal hydrogen atom of phenylacetylene, while the methanol-OH-proton forms a hydrogen bond with one of the catalyst's chlorine atoms. This step further stabilises the mechanism by $5.9 \mathrm{kcal} \mathrm{mol}^{-1}$.

In the next step, the reaction sequence diverges into two separate possible pathways. Further addition of another methanol molecule extends the hydrogen bonding network in the system. The mechanism is further stabilised by $10.8 \mathrm{kcal} \mathrm{mol}^{-1}$ with the formation 


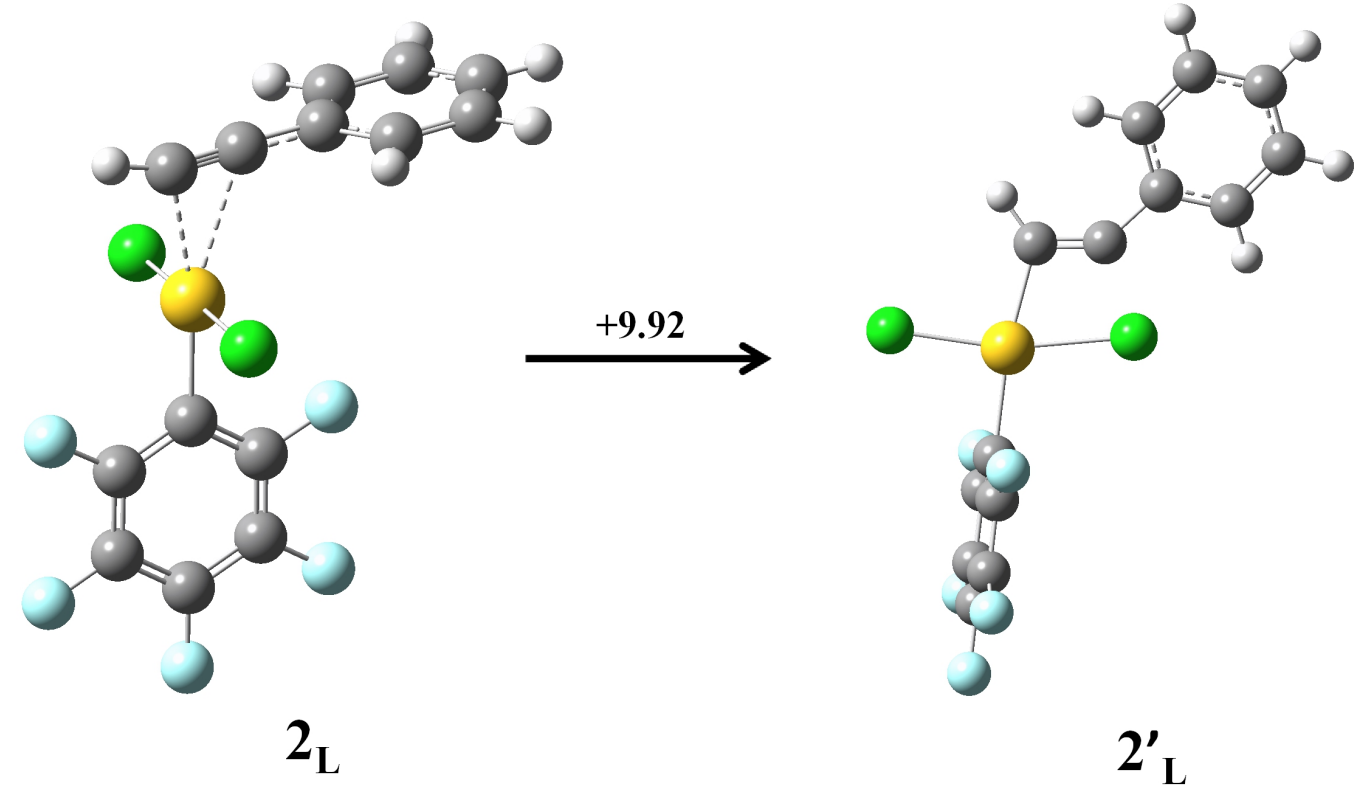

Figure 5.1 The perpendicular, $\mathbf{2}_{\mathrm{L}}$ and in-plane conformations, $\mathbf{2}_{\mathrm{L}}^{\prime}$ of $\mathrm{Au}\left(\mathrm{C}_{6} \mathrm{~F}_{5}\right) \mathrm{Cl}_{2}$-phenylacetylene activated complex. The energetic change from PBE0 calculation is given in $\mathrm{kcal} \mathrm{mol}^{-1}$.

of $\mathbf{4}_{\mathrm{L}}$ in one pathway while the other pathway is stabilised by $7.1 \mathrm{kcal} \mathrm{mol}^{-1}$ with the formation of $\mathbf{4}_{\mathrm{L}}^{\prime}$. Analogous to the reaction in Chapter 3, the $\mathbf{4}_{\mathrm{L}}^{\prime}$ structure has a methanol in position for nucleophilic attack while $\mathbf{4}_{\mathrm{L}}$ does not. From this point, the mechanism can either go through a nucleophilic attack or an extension of the hydrogen bonding network with further addition of a third methanol.

\subsubsection{Pathway 3MeOH-L}

In this pathway, the addition of a third methanol molecule yields $\mathbf{5}_{\mathrm{L}}$ which further stabilises the system by $4.9 \mathrm{kcal} \mathrm{mol}^{-1}$. The third methanol is now in position for nucleophilic attack. The activation barrier for $\mathbf{T S} \mathbf{A}_{\mathrm{L}}$ is $4.0 \mathrm{kcal} \mathrm{mol}^{-1}$ and the reaction yields the addition product $\mathbf{6}_{\mathrm{L}}$, which is $19.2 \mathrm{kcal} \mathrm{mol}^{-1}$ lower in energy with respect to the preceding stationary point. As the reaction proceeds from $\mathbf{5}_{\mathrm{L}}$ to $\mathbf{6}_{\mathrm{L}}$, the distance between the attacking oxygen atom and the central carbon atom decreases from $2.26 \AA$ in TS $\mathbf{A}_{\mathrm{L}}$ to $1.41 \AA$ in $\mathbf{6}_{\mathrm{L}}$. An increase of the $\mathrm{C}-\mathrm{C}$ bond length of the alkyne substrate from 


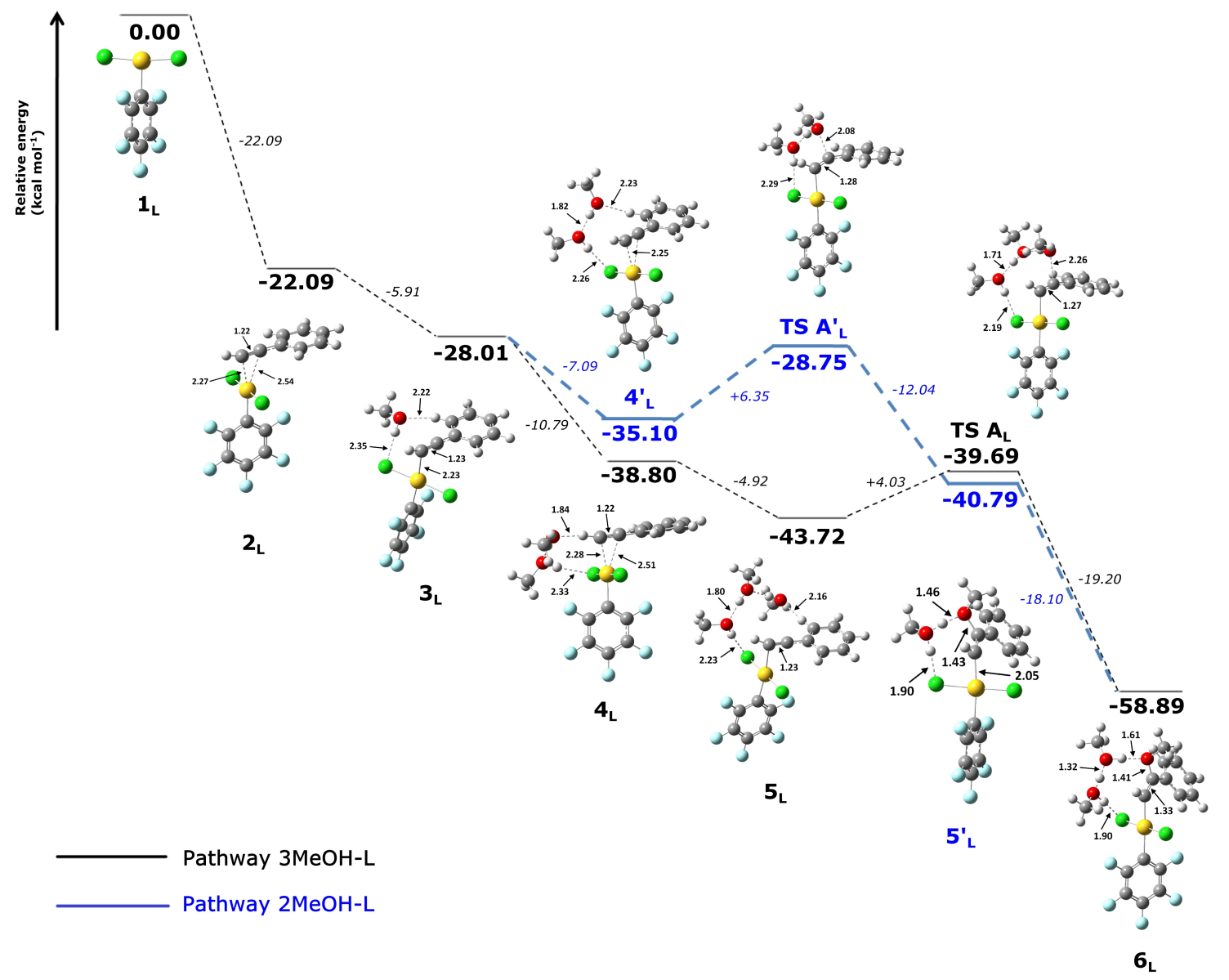

Figure 5.2 First part of the mechanism. Relative energies with respect to the starting materials from PBE0 calculations are given below structures $\mathbf{1}_{\mathrm{L}}$ to $\mathbf{6}_{\mathrm{L}}$ respectively while energetic changes with respect to the previous structures are given on the dotted lines that represent the reaction coordinate. The structural parameters are in $\AA$. All energies in $\mathrm{kcal} \mathrm{mol}^{-1}$.

$1.23 \AA$ to $1.33 \AA$ was also seen. $\mathbf{6}_{\mathrm{L}}$ intermediate represents the point in the mechanism where the two pathways converge again. More importanntly, it represents the stage of the mechanism where the initial nucleophilic attack has taken place, but the acidic $\mathrm{OH}$-proton of the attacking methanol has not migrated to the terminal carbon yet.

\subsubsection{Pathway 2MeOH-L}

In an alternative pathway (different from the one discussed in Section 5.1.1), the second methanol molecule can proceed with a nucleophilic attack straight after the 
formation of $\mathbf{4}_{\mathrm{L}}^{\prime}$ (this pathway is highlighted in blue in Figure 5.2). Similar to the results from Pathway $3 \mathrm{MeOH}-\mathrm{L}$, the activation barrier for this process is also very low $\left(6.4 \mathrm{kcal} \mathrm{mol}^{-1}\right)$. The geometry of the addition product $\mathbf{5}_{\mathrm{L}}^{\prime}$ is very similar to product $\mathbf{6}_{\mathrm{L}}$ discussed previously. Hydrogen migration does not take place out of $\mathbf{5}_{\mathrm{L}}^{\prime}$ as well. Hence, a third molecule of methanol is required to facilitate this last step of the first nucleophilic addition. As a result, the two possible reactions pathways converge at the formation of structure $6_{\mathrm{L}}$.

\subsubsection{Hydrogen migration and second nucleophilic attack}

Similar to the terminal alkyne in Chapter 3, the system undergoes structural rearrangement before the hydrogen migration occurs. The rearrangement of the methanols in $\mathbf{6}_{\mathrm{L}}$ causes the formation of $\mathbf{6} \mathbf{a}_{\mathrm{L}}$, which is $3.9 \mathrm{kcal} \mathrm{mol}^{-1}$ higher in energy. With the solvent methanols in position, the hydrogen migration proceeds with an activation barrier of only $1.4 \mathrm{kcal} \mathrm{mol}^{-1}$. This step demonstrates that the methoxy group, following the nucleophilic attack is deprotonated first before the hydrogen migrates to the terminal carbon. The mechanism then goes energetically downwards by $27.7 \mathrm{kcal} \mathrm{mol}^{-1}$ from the potential energy hill, forming $\mathbf{7} \mathbf{a}_{\mathrm{L}}$. The distance between the migrating hydrogen atom and terminal carbon atom decreases from $1.52 \AA$ in $\mathbf{T S} \mathbf{B}_{\mathrm{L}}$ to $1.08 \AA$ in $\mathbf{7} \mathbf{a}_{\mathrm{L}}$. As can be seen in the structure of $\mathbf{7} \mathbf{a}_{\mathrm{L}}$, the second methanol molecule is not in position for the second nucleophilic attack. The system then undergoes another structural rearrangement in preparation for the next nucleophilic attack to form $7_{\mathrm{L}}$, which is only $0.5 \mathrm{kcal} \mathrm{mol}^{-1}$ higher in energy than the preceding minimum. The transition state $\mathbf{T S} \mathbf{C}_{\mathrm{L}}$ sits at a $21.8 \mathrm{kcal} \mathrm{mol}^{-1}$ activation barrier with respect to the reactant. The second nucleophilic attack is somewhat higher in energy by $17.7 \mathrm{kcal} \mathrm{mol}^{-1}$ than the first attack. This is most likely due to the steric hindrance by the phenyl group along with the methoxy group introduced to the substrate earlier from the first nucleophilic attack. TS $\mathbf{C}_{\mathrm{L}}$ appears to be a late transition state that is structurally very similar to product $\mathbf{8}$, which is only 
$2.3 \mathrm{kcal} \mathrm{mol}^{-1}$ energetically more stable than $\mathbf{T S} \mathbf{C}_{\mathrm{L}}$ (shown in Figure 5.3). Similarly to the first nucleophilic attack, the nucleophile draws near to the reaction site at about $1.72 \AA$ in $\mathbf{T S} \mathbf{C}_{\mathrm{L}}$ and the distance decreases to $1.54 \AA$ as it forms $\mathrm{C}-\mathrm{O}$ bond in $\mathbf{8}_{\mathrm{L}}$. The hydrogen migration that finalises the reaction mechanism requires the largest activation energy $\left(\mathbf{T S} \mathbf{D}_{\mathrm{L}}\right.$ ) of $28.5 \mathrm{kcal} \mathrm{mol}^{-1}$, leading to the formation of $\mathbf{9}_{\mathrm{L}}$. The final ketal product is visible in structure $9_{\mathrm{L}}$, along with the solvent methanol molecule that facilitated the hydrogen migration in the previous step and the gold catalyst which is still weakly coordinated to the terminal carbon atom $(2.72 \AA)$. This final step of the catalytic cycle is predicted to be rate determining for the reaction. It is mainly due to the fact that there is a lack of coordination sites for the gold catalyst, leading to dissociation from the substrate at the same time as the hydrogen migrates. 


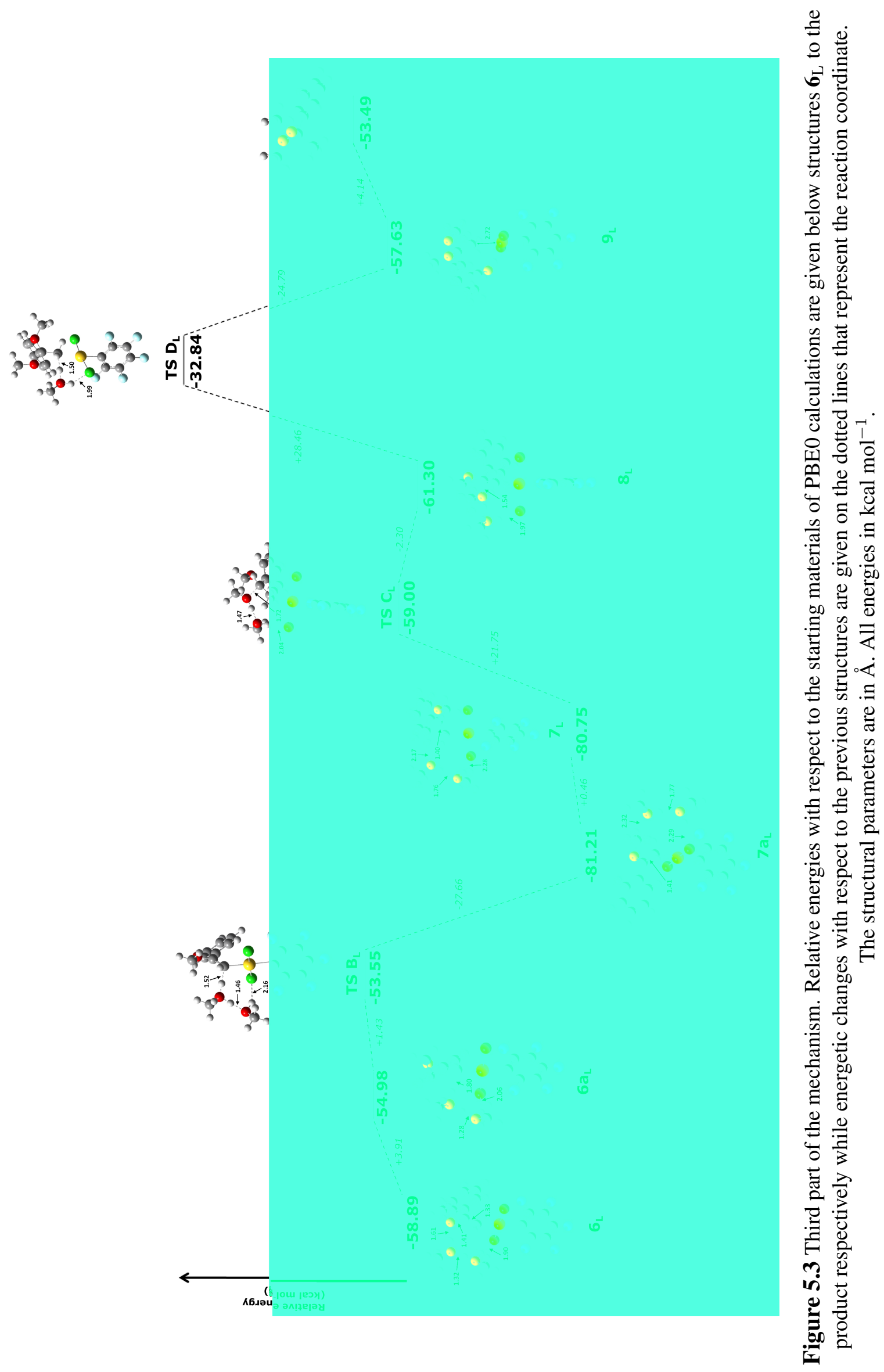


A point worthy of note is that the final step is endothermic in nature, where structure $\mathbf{9}_{\mathrm{L}}$ is $3.7 \mathrm{kcal} \mathrm{mol}^{-1}$ higher in energy than intermediate $\mathbf{8}_{\mathrm{L}}$. The final ketal product is released from the catalytic cycle and the cycle restarts itself with the elimination of the extra solvent methanol and gold catalyst in an endothermic process of only $4.1 \mathrm{kcal} \mathrm{mol}^{-1}$. However, the calculated Gibbs free energy change of this last step is actually exothermic by $18.0 \mathrm{kcal} \mathrm{mol}^{-1}$ which explains the feasibility of this catalytic system in experimental work. ${ }^{25}$ The thermodynamic properties of this reaction will be discussed in more details in Section 6.2. Both the activation barrier and the release of the final product from the catalytic cycle are energetically more favourable compared to the ones found in Chapter 3. This could be due to a stronger interaction between the terminal carbon and the migrating hydrogen. In Reaction 1 (Chapter 3), the NBO charges on the carbon atom was calculated as -0.84 a.u. while the migrating hydrogen possesses +0.46 a.u. charge. In this reaction, the NBO charges is -0.89 a.u. on the carbon and +0.49 a.u. on the migrating hydrogen. This implies that the attraction forces between the terminal carbon and migrating hydrogen is stronger in this reaction, promoting the formation of $\mathrm{C}-\mathrm{H}$ bond. The overall most probable mechanism for the reaction between methanol and phenylacetylene is depicted in Figure 5.4. 


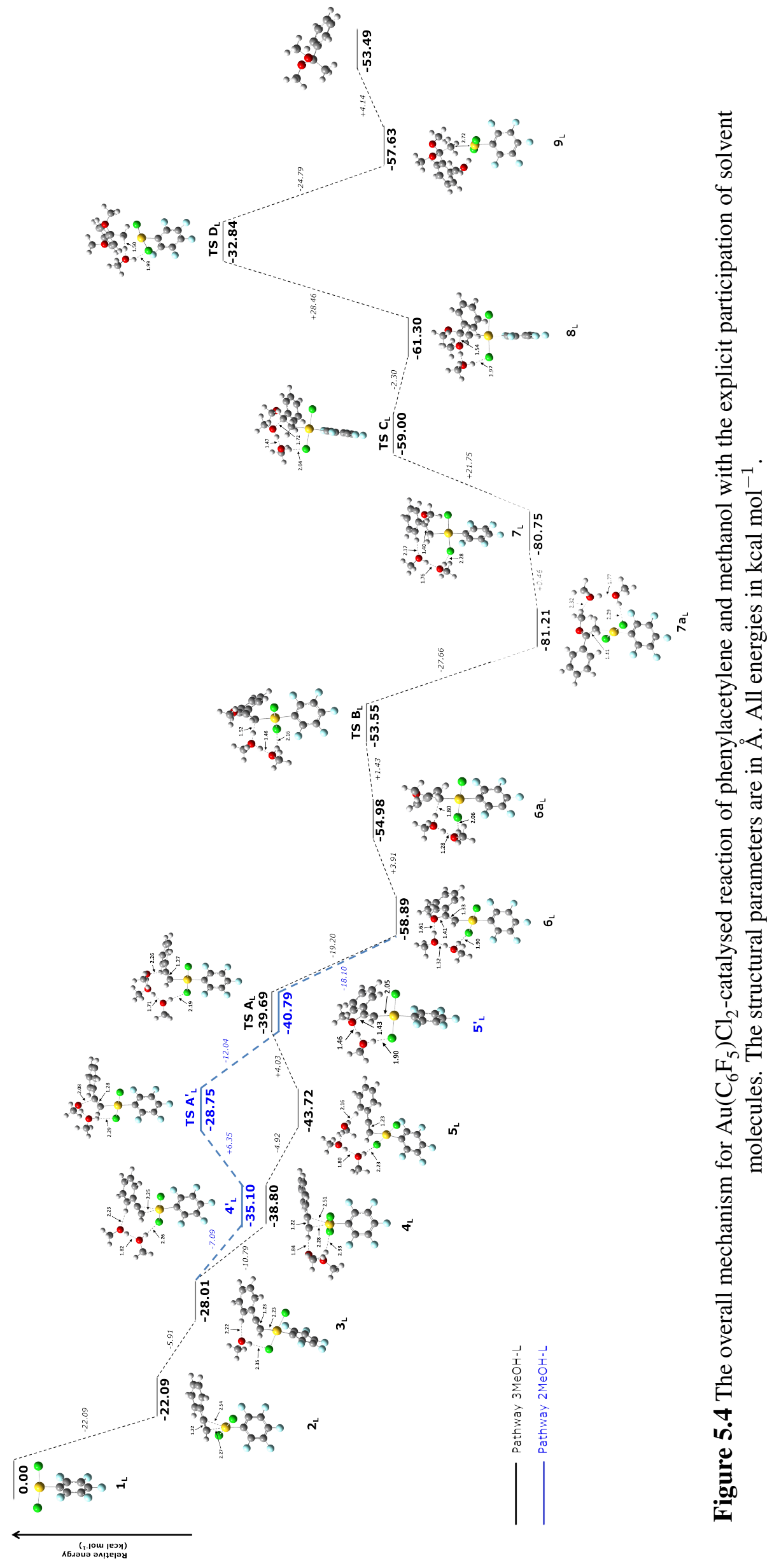




\subsection{Conclusions}

The results describe the reaction mechanism of the gold-catalysed ketal formation with phenylacetylene and methanol as starting materials. Each individual step in the mechanism highlights the importance of hydrogen bonding networks to facilitate the nucleophilic attacks as well as for the migrations of the hydrogen atoms after each addition step. Both nucleophilic attacks were predicted to proceed fast over low activation barriers with the first one having a lower activation barrier than the second one. This is due to the steric hindrance introduced by the methoxy group that was added in the first nucleophilic attack. The first hydrogen migration was found to occur in two steps; the deprotonation of methoxy group followed by the migration to the terminal carbon, and has a very low activation barrier. The second hydrogen migration on the other hand, requires a large activation energy and is predicted to be the rate determining step for the mechanism. The lack of a suitable coordination site for the remaining catalyst molecule may be the reason for this high activation barrier and relative destabilisation of the product. The entropic contribution to the system in the last step causes the Gibbs free energy to shift downwards significantly (discussed in Chapter 6.2). As a result, the release of the ketal product becomes a spontaneous process which contributes to the feasibility of this catalytic system in experimental work. ${ }^{25}$ 


\section{Chapter 6}

\section{Discussions: Comparison of the Three Reactions}

Three different reactions were investigated using the same level of theory as discussed in the previous chapters (Chapter 3-5). With the results obtained from mechanisms involving different substrates, comparison can be made to find out the factors that affect the gold-catalysed reaction mechanism for all the reactions under study.

\subsection{A comparison between terminal and internal alkynes}

Reaction 1 discussed in Chapter 3 is the reaction between propyne and methanol with $\mathrm{AuCl}_{3}$ as the catalyst. This is a representation of a reaction involving terminal alkynes. Reaction 2, on the other hand, is the reaction involving 4,4-dimethylpent-2-yne and methanol catalysed by $\mathrm{AuCl}_{3}$ (Chapter 4), which represents gold-catalysed transformations of internal alkynes. As a whole, both reactions proceed in similar processes which are double nucleophilic additions and followed by hydrogen migrations to complete the catalytic cycle. However, all individual steps in Reaction 1 have lower activation barriers compared to the corresponding steps in Reaction 2. This shows that internal alkynes do not react as effectively as terminal alkynes which is in agreement with previous experimental data. Another difference found between the two reactions is the hydrogen migration step found in Pathway $2 \mathrm{MeOH}$ that was observed in Reaction 2 but not in Reation 1. In general, the PES of such systems is flat due to the low energetic differences between the stationary points that are dominated by noncovalent interactions. Because of that, the transition state for the hydrogen migration step in Pathway $2 \mathrm{MeOH}$ of Reaction 1 could not be located due to its really flat energy surface, though it is suspected that the migration step should exist in that mechanism. It is also because of the higher activation 
barriers in Reaction 2 that its energy landscape is expected to be rougher compared to the energy surface of Reaction 1, which makes it possible to locate the hydrogen migration transition state (TS B1 ${ }_{\mathrm{m}}$ and $\mathbf{T S} \mathbf{B 2}{ }_{\mathrm{m}}{ }$ ) in Pathway $2 \mathrm{MeOH}$.

Another difference can be seen in how the catalyst coordinates to the substrate. Two conformations were found when the activated complex is formed - the in-plane and perpendicular orientations. The perpendicular orientation is more favoured in Reaction 2 while the in-plane orientation is more favoured in Reaction 1. In the perpendicular orientation, the catalyst is coordinated to the substrate in an $\eta^{2}$ fashion. On the other hand, the catalyst is found to coordinate to one of the unsaturated $\mathrm{C}$ atom in the in-plane orientation. Since the catalyst withdraws electron density from the substrate, it is important for the partial positive charge on the substrate to be stabilised by electron donating group(s). However, terminal alkynes lack this property. Therefore, the partial positive charge has to be stabilised by nearby chloro ligands of the catalyst, which can only be done in an in-plane orientation instead of the perpendicular orientation. This is evidently found in the NBO analysis of both orientations, which showed that the natural charge on the second carbon atom of propyne is reduced from 0.13 a.u. in the perpendicular orientation compared to -0.0034 a.u. in the in-plane orientation. Furthermore, a bonding orbital between the carbon atom and chloro ligand was found in the analysis, showing that this interaction stabilises the positive charge. This explains why the activated complex favours the in-plane orientation in Reaction 1.

In this study, two catalysts were used in the mechanistic investigations of the three reactions, which are $\mathrm{AuCl}_{3}$ and $\mathrm{Au}\left(\mathrm{C}_{6} \mathrm{~F}_{5}\right) \mathrm{Cl}_{2}$. If the two reactions of terminal alkynes (Reaction 1 and 3) were compared, it is shown that the activation barrier of the rate determining step in Reaction 3 is lower compared to that in Reaction 1. This suggests that the catalyst $\mathrm{Au}\left(\mathrm{C}_{6} \mathrm{~F}_{5}\right) \mathrm{Cl}_{2}$ is catalytically more active than $\mathrm{AuCl}_{3}$. However, the different alkynes used in these reactions can also affect the reactivity and the effects of the catalyst cannot be directly observed from the reactions. In light of this, we investigate 
the electronic properties of the activated complexes formed by the two catalysts with propyne and phenylacetylene. These properties were then compared between the two activated complexes formed from the same alkyne to study the effects of the catalysts. The NBO charge on the gold centre is +0.61 a.u. in the naked $\mathrm{AuCl}_{3}$ and +0.67 a.u. in $\mathrm{Au}\left(\mathrm{C}_{6} \mathrm{~F}_{5}\right) \mathrm{Cl}_{2}$, indicating that $\mathrm{C}_{6} \mathrm{~F}_{5}$ ligand is slightly more electron-withdrawing than $\mathrm{Cl}$ ligand. In principle, a more electrophilic metal centre withdraws more electron density from the $\pi$-bond, resulting in a larger decrease of charge on the metal and a larger increase of the charge on the reaction site compared to the less electrophilic metal centre.

When the activated complexes were formed, however, the charge on $\mathrm{AuCl}_{3}$ was observed to decrease more than $\mathrm{Au}\left(\mathrm{C}_{6} \mathrm{~F}_{5}\right) \mathrm{Cl}_{2}$. On top of that, the charge on the electrophilic-C atom is more positive when $\mathrm{AuCl}_{3}$ forms an activated complex with the alkynes under study. To confirm this, we tested the second nucleophilic attack of Reaction 3 when catalysed by $\mathrm{AuCl}_{3}$ and $\mathrm{Au}\left(\mathrm{C}_{6} \mathrm{~F}_{5}\right) \mathrm{Cl}_{2}$. It was found that this step has a lower activation energy when catalysed by $\mathrm{AuCl}_{3}$ by about $5 \mathrm{kcal} \mathrm{mol}^{-1}$. This suggests that while $\mathrm{Au}\left(\mathrm{C}_{6} \mathrm{~F}_{5}\right) \mathrm{Cl}_{2}$ is more electron-withdrawing, $\mathrm{AuCl}_{3}$ is catalytically more active, even though the effects are not very pronounced. A closer look at the NBO analysis of these complexes reveals that there exist metal- $\pi$ interactions with the pentafluorophenyl ring in $\mathrm{Au}\left(\mathrm{C}_{6} \mathrm{~F}_{5}\right) \mathrm{Cl}_{2}$, from the donor- $\pi$ bonds in the aromatic ring to the metal centre. This reduces the Lewis acidity of the catalyst and hence results in a less activated reaction. On a side note, it was also observed that the activated complexes of propyne and phenylacetylene prefer the in-plane orientation when complexed with $\mathrm{AuCl}_{3}$ while perpendicular orientation is preferred in $\mathrm{Au}\left(\mathrm{C}_{6} \mathrm{~F}_{5}\right) \mathrm{Cl}_{2}$ complexes. It is suspected that the $\mathrm{AuCl}_{3}$ catalyst, having higher Lewis acidity, has lower tendency for backbonding and adopt a $\sigma$-bonding interaction with alkynes (in-plane orientation). On the other hand, $\mathrm{Au}\left(\mathrm{C}_{6} \mathrm{~F}_{5}\right) \mathrm{Cl}_{2}$ has a higher tendency for backbonding that could strengthen the $\pi$ interaction with the alkyne and hence adopt the perpendicular orientation. This explains the higher stability of the in-plane structure in Reaction 1 and the more stable structure in Reaction 3 even though both reactants in these reactions are internal alkynes. 


\subsection{Thermodynamics of the reactions}

In order to relate the theoretical results with experimental data, the thermodynamics of the reactions in this work were taken into consideration. The three reactions under study have overall negative Gibbs free energy change, $\Delta G_{r}$ which means that the double nucleophilic addition of methanol to alkynes to form ketal product is spontaneous (Table 6.1). The Gibbs free energy changes for the internal alkynes (Reaction 2-M1 and M2) are less negative compared to the other reactions that were investigated. These values again justify the fact that internal alkynes do not react as effectively as terminal alkynes. Both Reaction 1 and 3 are very exergonic - highly negative Gibbs free energy change. In fact, Reaction 3 was carried out experimentally and was reported to proceed successfully. ${ }^{25}$

Table 6.1 Overall Gibbs free energy changes, $\Delta \mathrm{G}_{\mathrm{r}}$ of all reactions under study computed with PBE0 functional. All values are in $\mathrm{kcal} \mathrm{mol}^{-1}$.

\begin{tabular}{clr}
\hline & \multicolumn{1}{c}{ Reaction } & $\Delta \mathbf{G}_{\mathrm{r}}$ \\
\hline $\mathbf{1}$ & $\mathrm{HC} \equiv \mathrm{CCH}_{3}+2 \mathrm{CH}_{3} \mathrm{OH} \stackrel{+\mathrm{AuCl}_{3}}{\longrightarrow} \mathrm{CH}_{3} \mathrm{C}\left(\mathrm{OCH}_{3}\right)_{2} \mathrm{CH}_{3}$ & -24.26 \\
2-M1 & $\mathrm{CH}_{3} \mathrm{C} \equiv \mathrm{CC}\left(\mathrm{CH}_{3}\right)_{3}+2 \mathrm{CH}_{3} \mathrm{OH} \stackrel{+\mathrm{AuCl}_{3}}{\longrightarrow} \mathrm{CH}_{3} \mathrm{CH}_{2} \mathrm{C}\left(\mathrm{OCH}_{3}\right)_{2} \mathrm{C}\left(\mathrm{CH}_{3}\right)_{3}$ & -5.39 \\
$\mathbf{2 - M 2}$ & $\mathrm{CH}_{3} \mathrm{C} \equiv \mathrm{CC}\left(\mathrm{CH}_{3}\right)_{3}+2 \mathrm{CH}_{3} \mathrm{OH} \stackrel{+\mathrm{AuCl}_{3}}{\longrightarrow} \mathrm{CH}_{3} \mathrm{C}\left(\mathrm{OCH}_{3}\right)_{2} \mathrm{CH}_{2} \mathrm{C}\left(\mathrm{CH}_{3}\right)_{3}$ & -11.19 \\
$\mathbf{3}$ & $\mathrm{HC} \equiv \mathrm{C}\left(\mathrm{C}_{6} \mathrm{H}_{5}\right)+2 \mathrm{CH}_{3} \mathrm{OH} \stackrel{+\mathrm{Au}_{6}\left(\mathrm{C}_{5} \mathrm{~F}_{5}\right) \mathrm{Cl}_{2}}{\longrightarrow} \mathrm{CH}_{3} \mathrm{C}\left(\mathrm{OCH}_{3}\right)_{2}\left(\mathrm{C}_{6} \mathrm{H}_{5}\right)$ & -23.77 \\
\hline
\end{tabular}

Besides the overall Gibbs free energy change, it is also important to look at the feasibility of the last step of the reaction, which is the release of the final product by eliminating the catalyst and solvent molecule. Looking at just the (internal) energy of the system, this step is endothermic for the three reactions under study. To get a better representation of experimental work, the entropic contribution to the system is considered and the Gibbs free energy changes, $\Delta \mathrm{G}_{\text {release }}$ for this step were calculated and the values are tabulated in Table 6.2. By taking account of the entropic contribution, this step is shown to be spontaneous (negative Gibbs free energy change) across all the three reactions in this work. The release of the final ketal product is highly spontaneous for Reaction 3 as shown by the very negative Gibbs free energy change. The spontaneity of this step shows 
how readily the catalyst leaves the system to coordinate with another substrate and the catalytic cycle repeats itself.

Table 6.2 The energetic differences, $\Delta \mathrm{E}$ and Gibbs free energy changes, $\Delta \mathrm{G}_{\text {release }}$ for the release product by elimination of catalyst and extra solvent molecule (last step of reaction) computed with PBE0 functional. All values are in $\mathrm{kcal} \mathrm{mol}^{-1}$.

\begin{tabular}{|c|c|c|c|}
\hline & Reaction & $\Delta \mathbf{E}$ & $\Delta \mathbf{G}_{\text {release }}$ \\
\hline 1 & $\mathbf{9}_{\mathrm{s}} \longrightarrow \mathrm{CH}_{3} \mathrm{C}\left(\mathrm{OCH}_{3}\right)_{2} \mathrm{CH}_{3}+\mathrm{AuCl}_{3}+\mathrm{CH}_{3} \mathrm{OH}$ & +15.49 & -6.73 \\
\hline 2-M1 & $\begin{array}{l}\text { 9-1 } \mathrm{m} \longrightarrow \mathrm{CH}_{3} \mathrm{CH}_{2} \mathrm{C}\left(\mathrm{OCH}_{3}\right)_{2} \mathrm{C}\left(\mathrm{CH}_{3}\right)_{3}+\mathrm{AuCl}_{3}+ \\
\mathrm{CH}_{3} \mathrm{OH}\end{array}$ & +12.98 & -9.77 \\
\hline 2-M2 & $\begin{array}{l}\text { 9-2 } \mathrm{m} \longrightarrow \mathrm{CH}_{3} \mathrm{C}\left(\mathrm{OCH}_{3}\right)_{2} \mathrm{CH}_{2} \mathrm{C}\left(\mathrm{CH}_{3}\right)_{3}+\mathrm{AuCl}_{3}+ \\
\mathrm{CH}_{3} \mathrm{OH}\end{array}$ & +15.73 & -6.96 \\
\hline 3 & $\begin{array}{l}\mathbf{9}_{\mathrm{L}} \longrightarrow \mathrm{CH}_{3} \mathrm{C}\left(\mathrm{OCH}_{3}\right)_{2}\left(\mathrm{C}_{6} \mathrm{H}_{5}\right)+\mathrm{Au}\left(\mathrm{C}_{6} \mathrm{~F}_{5}\right) \mathrm{Cl}_{2}+ \\
\mathrm{CH}_{3} \mathrm{OH}\end{array}$ & +4.14 & -18.00 \\
\hline
\end{tabular}

Some $\mathrm{Au}(\mathrm{III})$ catalytic systems investigated experimentally (such as $\left.\mathrm{BzPPh} \mathrm{PPu}_{3}\left[\mathrm{C}_{6} \mathrm{~F}_{5}\right) \mathrm{Cl}_{3}\right]$ and $\mathrm{BzPPh}_{3}\left[\mathrm{Au}\left(\right.\right.$ mesityl) $\left.\mathrm{Cl}_{3}\right]$, where $\mathrm{Bz}$ stands for benzyl group) have shown difficulties converting the alkyne substrate to the ketal product successfully and result in a mixture of enol ether (termed as molecule 7 in all mechanisms) and ketal products (termed as molecule 9 in all reactions). ${ }^{25}$ This means that there is a competing pathway that prevent the formation of ketal product sucessfully. This is due to the chemoselectivity issue that is associated to alkynes. From the mechanisms that were investigated in this theoretical study, the reason for such observation is that the enol ether is energetically more stable compared to the ketal product. It is located in a deep potential well and the formation of this stable intermediate is thermodynamically favourable. In addition to that, the second nucleophilic attack that forms the ketal product often requires a high activation barrier. To get a better insight on this, the Gibbs free energy change, $\Delta G_{\text {enol }}$ for the formation of enol ether from the same substrate was calculated and tabulated in Table 6.3. The values show that the formation of enol ether is spontaneous.

The release of the enol ether product by elimination of catalyst and extra solvent molecules was also investigated. The energetic difference, $\Delta \mathrm{E}$ and Gibbs free energy change, $\Delta \mathrm{G}_{\text {enol-rel }}$ for the release of enol ether intermediate were calculated and the 
Table 6.3 Gibbs free energy changes, $\Delta \mathrm{G}_{\text {enol }}$ for the formation of enol ether computed with PBE0 functional. All values are in $\mathrm{kcal} \mathrm{mol}^{-1}$.

\begin{tabular}{clr}
\hline & \multicolumn{1}{c}{ Reaction } & $\Delta \mathbf{G}_{\text {enol }}$ \\
\hline $\mathbf{1}$ & $\mathrm{CH} \equiv \mathrm{CCH}_{3}+\mathrm{CH}_{3} \mathrm{OH} \stackrel{+\mathrm{AuCl}_{3}}{\longrightarrow} \mathrm{CH}_{2}=\mathrm{C}\left(\mathrm{OCH}_{3}\right) \mathrm{CH}_{3}$ & -18.93 \\
2-M1 & $\mathrm{CH}_{3} \mathrm{C} \equiv \mathrm{CC}\left(\mathrm{CH}_{3}\right)_{3}+\mathrm{CH}_{3} \mathrm{OH} \stackrel{+\mathrm{AuCl}_{3}}{\longrightarrow} \mathrm{CH}_{3} \mathrm{CH}=\mathrm{C}\left(\mathrm{OCH}_{3}\right) \mathrm{C}\left(\mathrm{CH}_{3}\right)_{3}$ & -9.66 \\
2-M2 & $\left.\mathrm{CH}_{3} \mathrm{C} \equiv \mathrm{CC}\left(\mathrm{CH}_{3}\right)_{3}+\mathrm{CH}_{3} \mathrm{OH} \stackrel{+\mathrm{AuCl}_{3}}{\longrightarrow} \mathrm{CH}_{3} \mathrm{C}\left(\mathrm{OCH}_{3}\right)=\mathrm{CHC}_{2} \mathrm{CH}_{3}\right)_{3}$ & -10.86 \\
$\mathbf{3}$ & $\mathrm{CH} \equiv \mathrm{C}\left(\mathrm{C}_{6} \mathrm{H}_{5}\right)+\mathrm{CH}_{3} \mathrm{OH} \stackrel{\left.+\mathrm{Au}_{6} \mathrm{C}_{5}\right) \mathrm{Cl}_{2}}{\longrightarrow} \mathrm{CH}_{2}=\mathrm{C}\left(\mathrm{OCH}_{3}\right)\left(\mathrm{C}_{6} \mathrm{H}_{5}\right)$ & -21.39 \\
\hline
\end{tabular}

values are shown in Table 6.4. The energetic differences for this alternative reaction are way too high for the reaction to be even feasible. In fact, even taking the entropic contribution into account, the Gibbs free energy change was seen to be very positive for the reactions considered in this work except for Reaction 3 and this mean that the release of enol ether from the catalytic cycle as an alternative product is not spontaneous.

Table 6.4 The energetic differences, $\Delta \mathrm{E}$ and Gibbs free energy changes, $\Delta \mathrm{G}_{\text {enol-rel }}$ for the release of the enol ether intermediate by elimination of catalyst and extra solvent molecule computed with PBE0 functional. All values are in $\mathrm{kcal} \mathrm{mol}^{-1}$.

\begin{tabular}{|c|c|c|c|}
\hline & Reaction & $\Delta \mathbf{E}$ & $\Delta \mathbf{G}_{\text {enol-rel }}$ \\
\hline 1 & $\mathbf{7}_{\mathrm{s}} \longrightarrow \mathrm{CH}_{2}=\mathrm{C}\left(\mathrm{OCH}_{3}\right) \mathrm{CH}_{3}+\mathrm{AuCl}_{3}+2 \mathrm{CH}_{3} \mathrm{OH}$ & +60.96 & +26.64 \\
\hline 2-M1 & $\begin{array}{l}\mathbf{7 - 1} \mathbf{m}_{\mathrm{m}} \longrightarrow \mathrm{CH}_{3} \mathrm{CH}=\mathrm{C}\left(\mathrm{OCH}_{3}\right) \mathrm{C}\left(\mathrm{CH}_{3}\right)_{3}+\mathrm{AuCl}_{3}+ \\
2 \mathrm{CH}_{3} \mathrm{OH}\end{array}$ & +55.85 & +20.07 \\
\hline 2-M2 & $\begin{array}{l}\mathbf{7 - 2} \mathrm{m}_{\mathrm{m}} \longrightarrow \mathrm{CH}_{3} \mathrm{C}\left(\mathrm{OCH}_{3}\right)=\mathrm{CHC}\left(\mathrm{CH}_{3}\right)_{3}+\mathrm{AuCl}_{3}+ \\
2 \mathrm{CH}_{3} \mathrm{OH}\end{array}$ & +57.71 & +22.25 \\
\hline 3 & $\begin{array}{l}7_{\mathrm{L}} \longrightarrow \mathrm{CH}_{2}=\mathrm{C}\left(\mathrm{OCH}_{3}\right)\left(\mathrm{C}_{6} \mathrm{H}_{5}\right)+\mathrm{Au}\left(\mathrm{C}_{6} \mathrm{~F}_{5}\right) \mathrm{Cl}_{2}+ \\
2 \mathrm{CH}_{3} \mathrm{OH}\end{array}$ & +43.96 & +7.49 \\
\hline
\end{tabular}

As a summary, the outcome of the reaction can be predicted from the thermodynamic quantities calculated. Both Reaction 1 and 3 are very exergonic, indicating that the formation of the ketal product is highly favourable. The possibility of the mechanism terminating with the formation of the enol ether intermediate was also considered. Both reactions have highly negative $\Delta \mathrm{G}_{\text {enol }}\left(-18.9 \mathrm{kcal} \mathrm{mol}^{-1}\right.$ and $\left.-21.4 \mathrm{kcal} \mathrm{mol}^{-1}\right)$ but the release of enol ether from the catalytic cycle was found to be highly not spontaneous $\left(\Delta \mathrm{G}_{\text {enol-rel }}=+26.6 \mathrm{kcal} \mathrm{mol}^{-1}\right)$ for Reaction 1 . Therefore, it is expected that Reaction 1 
would only produce the ketal as the final product, experimentally. The $\Delta \mathrm{G}_{\text {enol-rel }}$ calculated in Reaction 3, on the other hand, is surprisingly low $\left(+7.5 \mathrm{kcal} \mathrm{mol}^{-1}\right)$ compared to the other reactions. The possibility of termination at the enol ether intermediate cannot be completely disregarded. In fact, this reaction was previously conducted experimentally by Laguna and coworkers ${ }^{25}$ and it was reported that the reaction yields a 1:1 mixture of ketal and enol ether products. This is in line with the calculated

values of $\Delta \mathrm{G}_{\mathrm{r}}\left(-23.8 \mathrm{kcal} \mathrm{mol}^{-1}\right)$ and $\Delta \mathrm{G}_{\mathrm{enol}}\left(-21.4 \mathrm{kcal} \mathrm{mol}^{-1}\right)$ for Reaction 3 which are very similar, indicating that the competing reaction channels are equally dominant, resulting in a mixture of products.

In the case of Reaction 2-M1, the calculated Gibbs free energy change for both the formation of enol ether and ketal products are feasible but the enol ether formation is predicted to be more favourable. In contrast, the release of ketal from the catalytic cycle is calculated to be more favourable than enol ether. Under these circumstances, it is difficult to predict in confidence how the reaction would go experimentally but it is expected that a mixture of products would be the outcome. The mixture of products could be a result of a kinetically controlled environment. The second nucleophilic attack to form the ketal requires a high activation barrier in M1. Although the Gibbs free energy change, $\Delta \mathrm{G}_{\text {enol-rel }}$ for the release of enol ether as the product is highly not feasible, this energy requirement might be compensated by the energy release when a free catalyst coordinates with another substrate to repeat the cycle. As for Reaction 2-M2, the calculated thermodynamic quantities show a similar pattern as Reaction 1, implying that the reaction would most likely yield only the ketal product experimentally.

\subsection{Reaction kinetics}

The kinetics of the reactions were studied. Using the information obtained from the mechanisms, the rate constants for each individual step in all the reaction mechanisms 
under study were calculated based on the assumptions and formulas in Chapter 2.5. The values are tabulated in Tables 6.5-6.8. Using the values of the calculated rate constants, we are able to produce plots of concentrations against time using the Mathematica 10 software package ${ }^{148}$ by solving the differential equations of the concentration of each species simultaneously. Here, we show an example of how the differential equations are derived for Reaction 1.

$$
3_{\mathrm{s}} \longrightarrow 4_{\mathrm{s}} \longrightarrow 5_{\mathrm{s}} \stackrel{\mathrm{k}_{\mathrm{A}}}{\longrightarrow} 6_{\mathrm{s}} \longrightarrow 6 \mathrm{a}_{\mathrm{s}} \stackrel{\mathrm{k}_{\mathrm{B}}}{\longrightarrow} 7 \mathrm{a}_{\mathrm{s}} \longrightarrow 7_{\mathrm{s}} \stackrel{\mathrm{k}_{\mathrm{C}}}{\longrightarrow} 8_{\mathrm{s}} \stackrel{\mathrm{k}_{\mathrm{D}}}{\longrightarrow} 9_{\mathrm{s}}
$$

and at the same time,

$$
\begin{aligned}
& 3_{\mathrm{s}} \longrightarrow 4^{\prime}{ }_{\mathrm{s}} \stackrel{\mathrm{k}_{\mathrm{A}^{\prime}}}{\longrightarrow} 5^{\prime}{ }_{\mathrm{s}} \longrightarrow 6_{\mathrm{s}} \stackrel{\mathrm{k}_{\mathrm{B}^{\prime}}}{\longrightarrow} 7^{\prime}{ }_{\mathrm{s}} \longrightarrow 7_{\mathrm{s}} \stackrel{\mathrm{k}_{\mathrm{C}}}{\longrightarrow} 8_{\mathrm{s}} \stackrel{\mathrm{k}_{\mathrm{D}}}{\longrightarrow} 9_{\mathrm{s}} \\
& \frac{\mathrm{d}\left[3_{\mathrm{s}}\right]}{\mathrm{d} t}=-k_{\mathrm{A}^{\prime}}\left[4_{\mathrm{s}}^{\prime}\right]-k_{\mathrm{A}}\left[5_{\mathrm{s}}\right] \\
& \frac{\mathrm{d}\left[6_{\mathrm{s}}\right]}{\mathrm{d} t}=k_{\mathrm{A}^{\prime}}\left[4_{\mathrm{s}}^{\prime}\right]+k_{\mathrm{A}}\left[5_{\mathrm{s}}\right]-k_{\mathrm{B}}\left[6_{\mathrm{s}}\right]-k_{\mathrm{B}^{\prime}}\left[6_{\mathrm{s}}\right] \\
& \frac{\mathrm{d}\left[7_{\mathrm{s}}\right]}{\mathrm{d} t}=k_{\mathrm{B}}\left[6_{\mathrm{s}}\right]+k_{\mathrm{B}^{\prime}}\left[6_{\mathrm{s}}\right]-k_{\mathrm{C}}\left[7_{\mathrm{s}}\right] \\
& \frac{\mathrm{d}\left[8_{\mathrm{s}}\right]}{\mathrm{d} t}=k_{\mathrm{C}}\left[7_{\mathrm{s}}\right]-k_{\mathrm{D}}\left[8_{\mathrm{s}}\right] \\
& \frac{\mathrm{d}\left[9_{\mathrm{s}}\right]}{\mathrm{d} t}=k_{\mathrm{D}}\left[8_{\mathrm{s}}\right]
\end{aligned}
$$

A few assumptions were made to plot the graphs.

1) The 'barrierless' transformations in the mechanism, e.g. $\mathbf{7} \mathbf{a}_{\mathrm{s}}$ to $\mathbf{7}_{\mathrm{s}}$ or $\mathbf{3}_{\mathrm{s}}$ to $\mathbf{4}_{\mathrm{s}}$ are assumed to proceed with $100 \%$ conversion. This means that the concentrations of the preceding and succeeding minimum are the same. This also applies to minima such as $\mathbf{4}_{\mathrm{s}}^{\prime}$ and $\mathbf{5}_{\mathrm{s}}$ where they both have the same concentration as $\mathbf{3}_{\mathrm{s}}$. These two minima represents the point just before the first nucleophilic attack. In other words, this assumption also groups the minima according to their position/role in the mechanism, i.e. the point before/after nucleophilic attack/hydrogen migration. 
2) The values of rate constants calculated are in a wide range. Using these values, it is difficult to visualize all the curves on the same axes and this fails to show a good projection of concentration with respect to time. In order to rectify this problem without compromising the data, a set of values is used to relatively represent the calculated rate constants in the plots. The value of 1 is assigned to the highest rate constant of a reaction while the value of 0.001 is assigned to the lowest rate constant of that particular reaction. This creates a relative scale with respect to the range of rate constant values, in which the position of the rate constants in the scale can be determined with respect to the maximum and minimum. As a result, values in the range of 0.001 to 1 can be assigned to each rate constant based on that scale. The calculations are explained in details in Appendix A.

Table 6.5 Rate constant values, $k\left(\mathrm{~s}^{-1}\right)$ and the corresponding relative values, $k^{\prime}\left(\mathrm{s}^{-1}\right)$ for Reaction 1.

\begin{tabular}{lll}
\hline Steps & $k$ & $k^{\prime}$ \\
\hline $\mathrm{A}$ & $1.545 \times 10^{9}$ & 0.6 \\
$\mathrm{~A}^{\prime}$ & $9.854 \times 10^{8}$ & 0.6 \\
$\mathrm{~B}$ & $1.004 \times 10^{11}$ & 1 \\
$\mathrm{~B}^{\prime}$ & $2.313 \times 10^{5}$ & 0.2 \\
$\mathrm{C}$ & $5.451 \times 10^{3}$ & 0.1 \\
$\mathrm{D}$ & $1.181 \times 10^{-15}$ & 0.001 \\
\hline
\end{tabular}

Table 6.6 Rate constant values, $k\left(\mathrm{~s}^{-1}\right)$ and the corresponding relative values, $k^{\prime}\left(\mathrm{s}^{-1}\right)$ for Reaction 2-M1.

\begin{tabular}{lll}
\hline Steps & $k$ & $k^{\prime}$ \\
\hline $\mathrm{A}$ & $9.068 \times 10^{4}$ & 0.1 \\
$\mathrm{~A}^{\prime}$ & $4.558 \times 10^{2}$ & 0.06 \\
$\mathrm{~B}$ & $1.159 \times 10^{3}$ & 0.07 \\
$\mathrm{~B}^{\prime}$ & $2.910 \times 10^{12}$ & 1 \\
$\mathrm{C}$ & $5.059 \times 10^{-10}$ & 0.002 \\
$\mathrm{D}$ & $3.190 \times 10^{-13}$ & 0.001 \\
\hline
\end{tabular}


Table 6.7 Rate constant values, $k\left(\mathrm{~s}^{-1}\right)$ and the corresponding relative values, $k^{\prime}\left(\mathrm{s}^{-1}\right)$ for Reaction 2-M2.

\begin{tabular}{lll}
\hline Steps & $k$ & $k^{\prime}$ \\
\hline $\mathrm{A}$ & $1.971 \times 10^{2}$ & 0.2 \\
$\mathrm{~A}^{\prime}$ & $1.177 \times 10^{2}$ & 0.2 \\
$\mathrm{~B}$ & $7.025 \times 10^{5}$ & 0.3 \\
$\mathrm{~B}^{\prime}$ & $3.619 \times 10^{11}$ & 1 \\
$\mathrm{C}$ & $4.906 \times 10^{2}$ & 0.2 \\
$\mathrm{D}$ & $1.276 \times 10^{-26}$ & 0.001 \\
\hline
\end{tabular}

Table 6.8 Rate constant values, $k\left(\mathrm{~s}^{-1}\right)$ and the corresponding relative values, $k^{\prime}\left(\mathrm{s}^{-1}\right)$ for Reaction 3.

\begin{tabular}{lll}
\hline Steps & $k$ & $k^{\prime}$ \\
\hline $\mathrm{A}$ & $8.687 \times 10^{7}$ & 0.3 \\
$\mathrm{~A}^{\prime}$ & $1.634 \times 10^{5}$ & 0.1 \\
$\mathrm{~B}$ & $3.106 \times 10^{11}$ & 1 \\
$\mathrm{C}$ & $3.053 \times 10^{-07}$ & 0.002 \\
$\mathrm{D}^{\mathrm{a}}$ & $2.893 \times 10^{-09}$ & 0.001 \\
\hline \multicolumn{3}{c}{ a } \\
\multicolumn{3}{c}{ The $q_{\text {vib }}$ for this step is calculated } \\
at double- $\zeta$ quality.
\end{tabular}

The graphs in Figure 6.1-Figure 6.4 represent projections of how the concentration of each species changes with respect to time, which can be used to predict experimental outcomes. From the plots of all the reactions, it can be seen that the concentrations of the species (molecules 4, $\mathbf{5}$ and $\mathbf{6}$ ) before the second nucleophilic attack decrease significantly in a short amount of time, indicating that the rate of consumption is very high. This also means that it would be difficult to detect these intermediates in experiments due to their short life span. From the thermodynamics results in Section 6.2, the data shows that Reaction 1 and Reaction 2-M2 would most likely proceed without a mixture of enol ether (7) and ketal (9) products. This is supported by the Arrhenius plots of these reactions. It can be seen that the concentration of enol ether has already started to decrease in the earlier stage of Reaction 1 and Reaction 2-M2, indicated by the yellow line in the smaller graphs in Figure 6.1 and 6.3. This means that the second nucleophilic attacks in these two reactions proceed at a higher rate and the reactions would be complete with the formation of ketal. 
On the other hand, the consumption of enol ether in Reaction 2-M1 and Reaction 3 is shown to proceed at a lower rate as depicted in Figure 6.2 and 6.4. This indicates that the enol ether is consumed at a relatively lower rate and there is a possibility for the catalyst to be deactivated before all of the enol ether is converted to ketal product, hence resulting in a mixture of products. This is also in agreement with our claim from the thermodynamic perspectives that both Reaction 2-M1 and Reaction 3 would most likely result in a mixture of enol ether and ketal products. It would be interesting to compare the experimental rate of deactivation of catalyst against the rate at which the second nucleophilic attack proceeds. From this, the reaction can be controlled so that it can reach full completion to form ketal as the only product. This leads us back to the root of most catalyst designs which is the stability of catalyst, discussed in Chapter 1.3. A stable catalyst has a longer lifetime and thus, be able to continue catalysing the reaction to its full completion.

In all of the graphs plotted, the ketal products (represented by the blue lines) are formed at a very slow rate mainly due to the high activation barrier for the last migration step. Increasing the temperature of reaction can increase the rate of formation of ketal but this also increases the possibility of the release of enol ether and render the reaction incomplete. This can be seen from the values in Table 6.4 which will decrease in higher temperature as the entropic effects increase. The better approach instead is to develop a more active catalyst that can reduce the activation barrier of the rate determining step. 


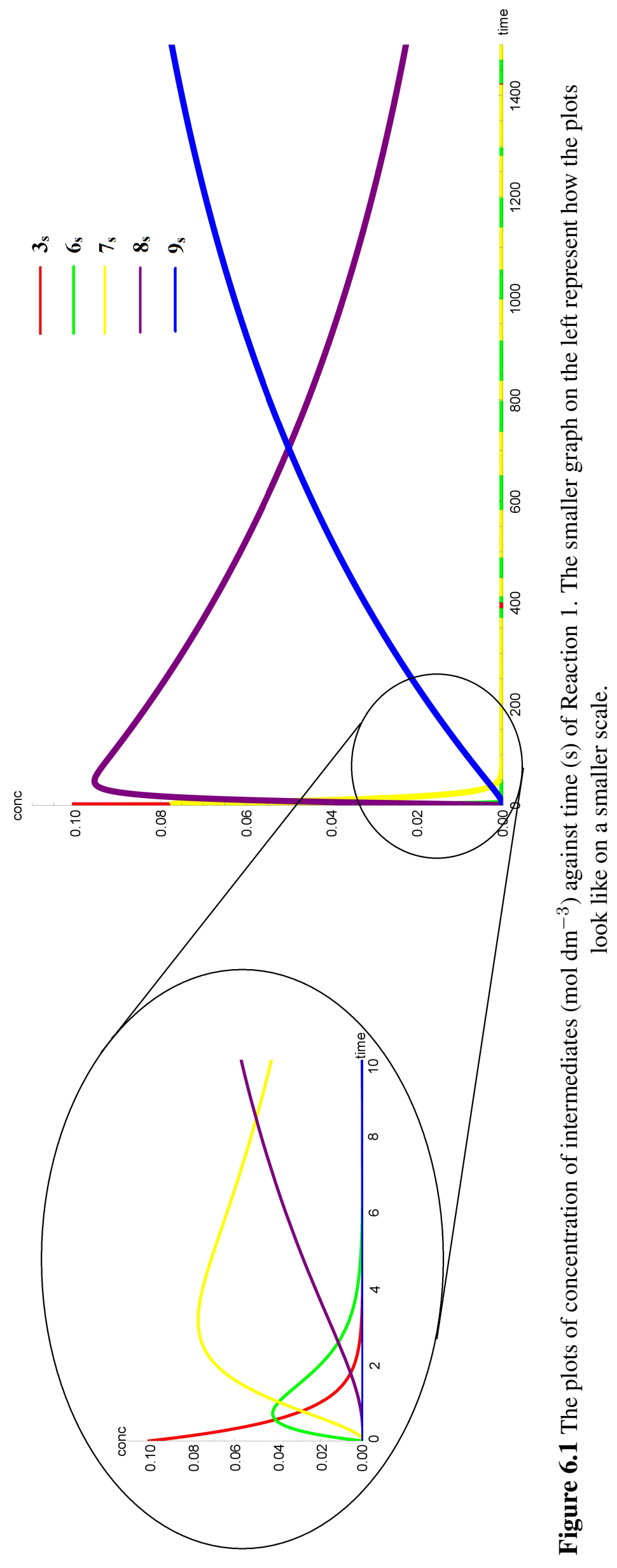




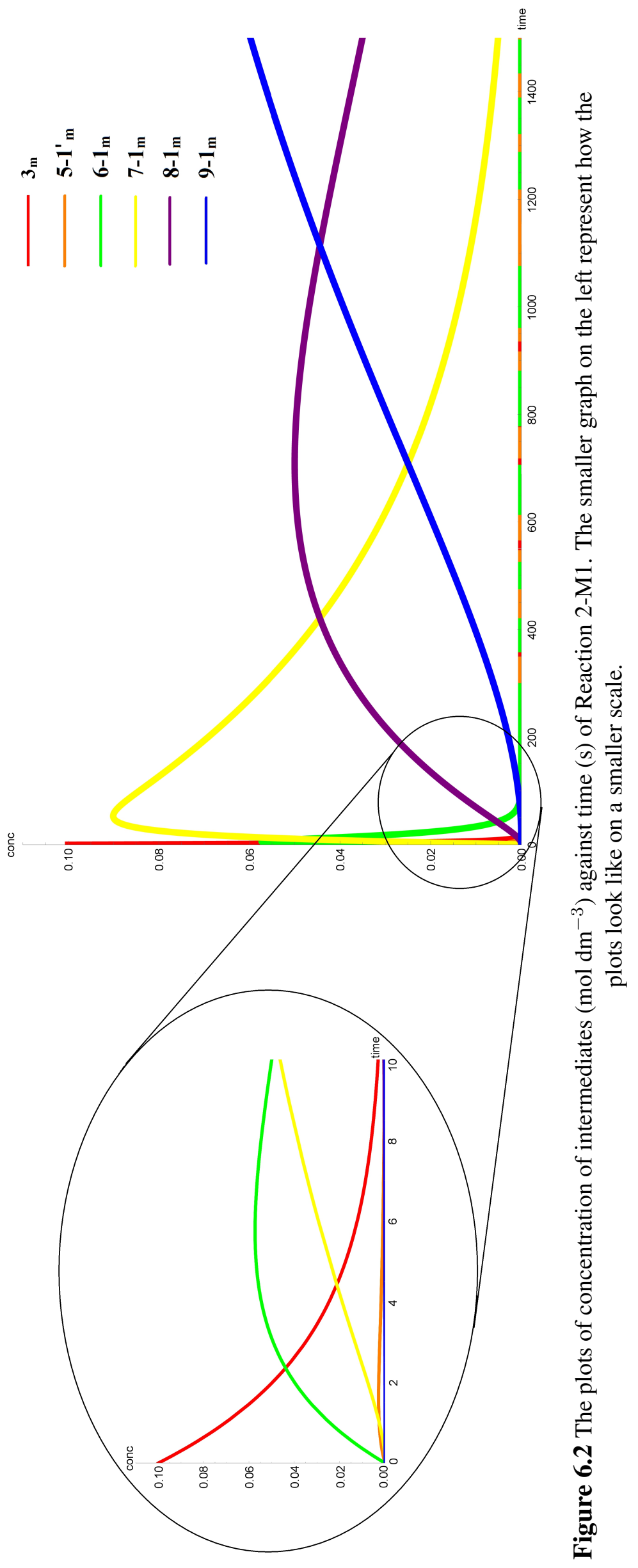




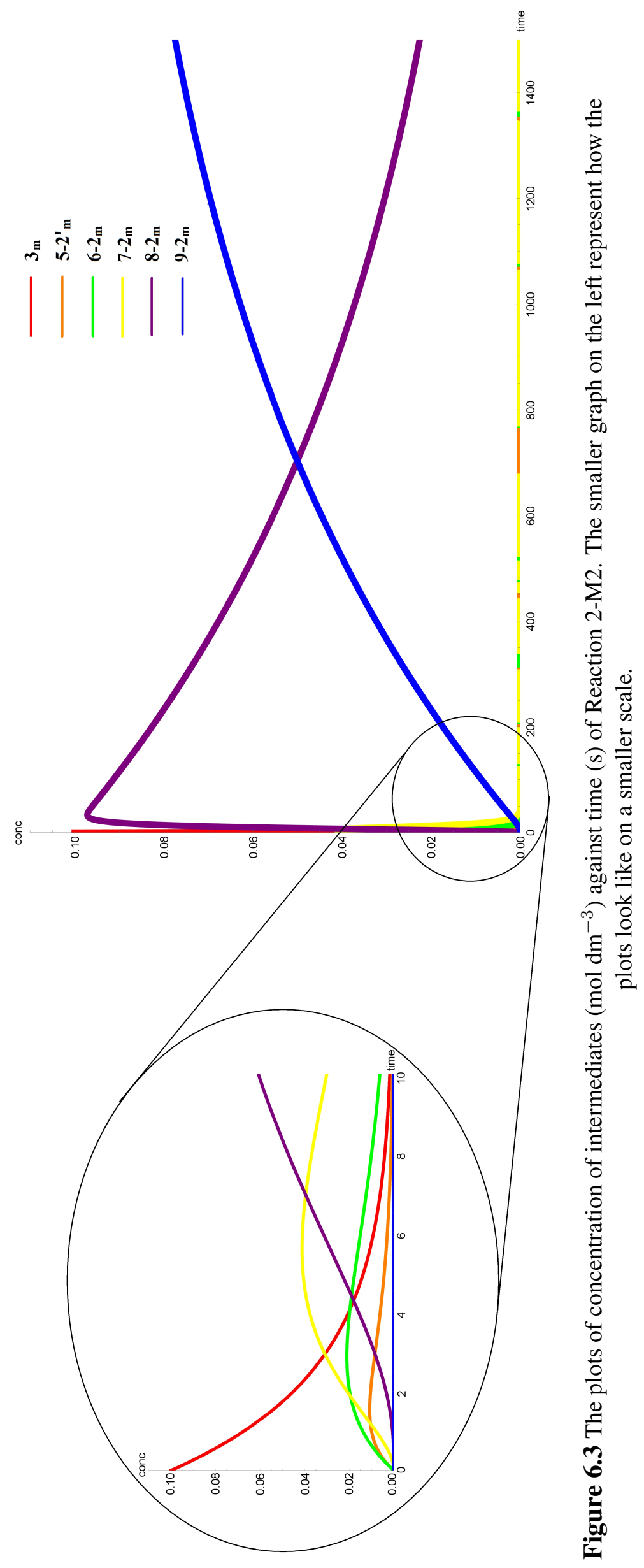




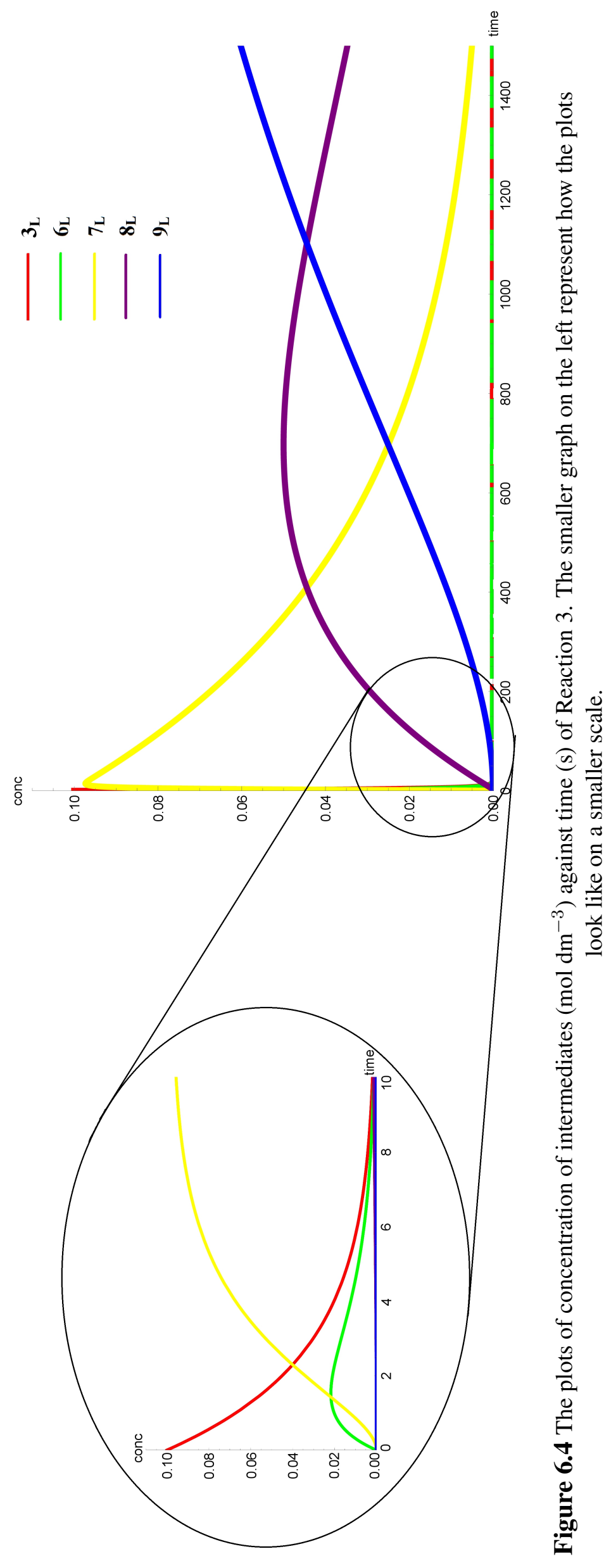




\section{Chapter 7}

\section{Summary and Outlook}

The study provides a detailed and thorough mechanism for the Au(III)-catalysed hydroalkoxylation of various alkynes. The mechanisms found for all reactions under study show the importance of hydrogen bonding network and the participation of solvent molecules in each step leading to the formation of products. The rate determining step was found to be the last hydrogen migration for the reactions studied. In Reaction 1 (Chapter 3), two hydrogen migration steps were found. In one of the hydrogen migration, the solvents form a 'track' within the hydrogen bonding network to transfer the hydrogen from one solvent to another until it finally reaches the terminal carbon. The other migration step, which requires lower activation barrier, involves a deprotonation from the methoxy group before the migration begins. These two steps can be clearly seen in Figure 3.3.

Next, the study extends to the mechanistic investigation involving internal alkyne (Chapter 4). A comparison between internal and terminal alkynes was made to give more insight into the different behaviours of substrates in the reactions. Terminal alkynes were experimentally determined to proceed faster in the reaction compared to internal alkynes and this is in good agreement with our results. This is mainly due to the lower activation barriers of each individual step in the mechanism. The complexity of reactions involving internal alkynes was also demonstrated in the study. As a result of the regioselectivity issue in asymmetric internal alkynes, the reaction yields a mixture of products. In light of this, it was found that one of the mechanisms is kinetically more favourable while the other is thermodynamically more favourable.

For the last reaction model (Chapter 5), we attempt to test if an experimentally determined system would follow the same mechanism found for the first two reactions. As it turns 
out, this reaction also follows the same straightforward processes, which begins with the first nucleophilic attack, followed by a hydrogen migration to complete the process and these steps are repeated in the second nucleophilic attack. The effect of different ligands in the catalyst was also investigated and the results show that ligands with higher Lewis acidity improve the reactivity of the catalyst. In an effort to predict the experimental outcome of the reaction, the thermodynamics and kinetics of the reactions were studied. Reaction 1 and Reaction 2-M2 were predicted to proceed to full completion with the formation of ketal as the only product. On the other hand, Reaction 2-M1 and Reaction 3 were predicted to yield a mixture of products of enol ether and ketal.

Several adjustments can be implemented to improve the current theoretical method of this study. One of the ways is to include dispersion correction in the method to better account for the weak interactions especially the hydrogen bonding network in the system. DFT is infamously known to perform poorly in calculating dispersion forces between molecules at large distances. However, for the systems in the current work, the hydrogen bonding network is small and at close distances with each other, hence we would still expect the functional without dispersion correction to perform relatively well in the calculations. Another way to improve the results is to perform the calculations in solution phase using the PCM model as discussed in Chapter 2. In a highly charged reaction model, the PCM approach would yield a significant improvement to the energies and consequently produce a corrected energy landscape of the reaction mechanism. However, the system and solvent used in the current study are neutrally charged and the PCM approach in this case often fails to show the significance of the solvent effects. Instead, the solvent effects are taken into account by explicitly including the solvent molecules in the calculations.

With the information obtained in this work, we hope to be able to improve future synthetic processes of $\mathrm{Au}(\mathrm{III})$-catalysed reactions. Participation of solvent molecules is important in the mechanism and thus, it is important to choose a suitable protic solvent to improve the hydrogen bonding network in the system and assists the reaction more effectively. 
For example, the use of $t$-butanol could help the reaction as it is a good base to assist the hydrogen migration. At the same time, bulky tertiary alcohols do not react in this reaction which means that $t$-butanol would only act as the assisting solvent molecule without reacting with the substrate to prevent a mixture of products. Most experimental works showed that most successful Au(III) catalysts bear NHC-ligands, halide ligands and phosphorus-containing ligands. It would be interesting to see how O-contaning ligands fare with the other ligands as they have the potential to form a better hydrogen bonding with the solvents. At the same time, the design of catalysts should also maintain the Lewis acidity of the catalyst to ensure a good activation of the $\mathrm{C}-\mathrm{C}$ triple bonds of alkynes. Ligands with electron deficient central atoms such as boron-centered molecules $\left(\mathrm{BH}_{3}\right.$, $\mathrm{BF}_{3}$, etc.) are useful in increasing the Lewis acidity of the complex. 


\section{Appendix A}

\section{Calculation of relative rate constant values, $k^{\prime}$}

The values of rate constants of each elementary step in the reactions studied are in a wide range. For example, the results for Reaction 1 vary over four orders of magnitude (See Table 6.5). Due to the variation in these values, it becomes impossible to visualize all Arrhenius plots on the same axis if the absolute rate constant values are used. In fact, transition state theory which is adopted in this study, is known to be less successful in calculating the absolute reaction rate constants as it requires the precise knowledge of the potential energy surfaces. Instead, we are more interested in the relative rate constants to see how fast one elementary step progresses with respect to the other elementary steps in the reaction. In this case, we answer the question with "Step A is 10 times faster than Step B" as opposed to "Step A is 20 minutes faster than Step B". In order to account for the large range of values and to project the results in the way we are interested in, the set of rate constants is represented on a logarithmic scale as depicted in the figure below.

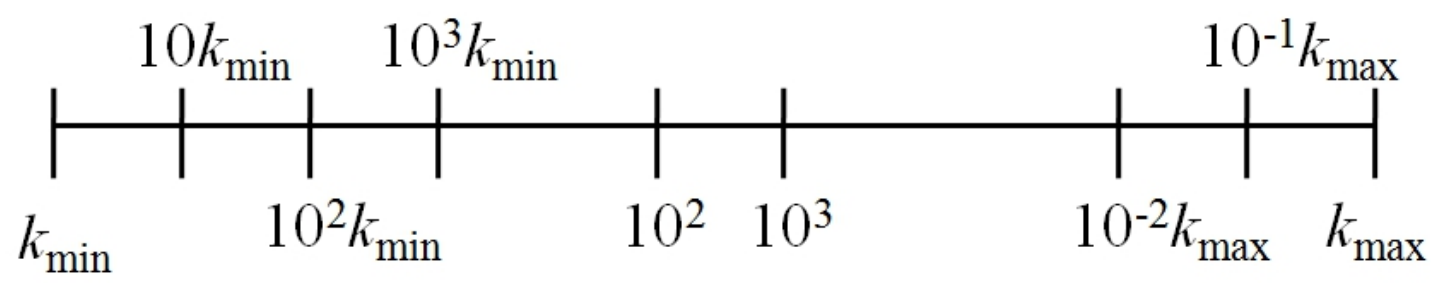

Figure A.1 Rate constant values represented on a logarithmic scale with $k_{\min }$ being the lowest value while $k_{\max }$ being the largest value in the set.

In order to plot the Arrhenius curves on the same axes and at the same time maintain a good representation of the calculated 'absolute' rate constants, a number of steps is followed to get a good projection of the 'absolute' values on a smaller scale which is termed as $k^{\prime}$ in the discussion (Chapter 6.3). The values used in the following examples are calculated for Reaction 1 (See Table 6.5). The procedures are as follows: 
1) A set of $k^{\prime}$ values are required and should be of small values so that all the curves can fit into the axes. The pre-determined range is from 0.001 to 1 .

2) The $k_{\min }$ and $k_{\max }$ values are identified and they are automatically assigned the $k^{\prime}$ values of 0.001 and 1 , respectively. E.g. the value $1.181 \times 10^{-15}$ is the smallest in the group and is assigned $k^{\prime}$ value of 0.001 while $1.004 \times 10^{11}$, being the largest value in the set is assigned the value 1 .

\begin{tabular}{ll}
\hline Steps & $k$ \\
\hline $\mathrm{A}$ & $1.545 \times 10^{9}$ \\
$\mathrm{~A}^{\prime}$ & $9.854 \times 10^{8}$ \\
$\mathrm{~B}$ & $1.004 \times 10^{11} \longrightarrow k_{\max }$ \\
$\mathrm{B}^{\prime}$ & $2.313 \times 10^{5}$ \\
$\mathrm{C}$ & $5.451 \times 10^{3}$ \\
$\mathrm{D}$ & $1.181 \times 10^{-15} \longrightarrow k_{\text {min }}$ \\
\hline
\end{tabular}

Figure A.2 The assignment of $k_{\min }$ and $k_{\max }$.

3) In the following step, the $k_{\min }$ and $k_{\max }$ values are converted to their corresponding logarithmic values and the range is determined as $d_{k}$. The same applies to $k^{\prime}$ and the range is determined as $d_{k^{\prime}}$.

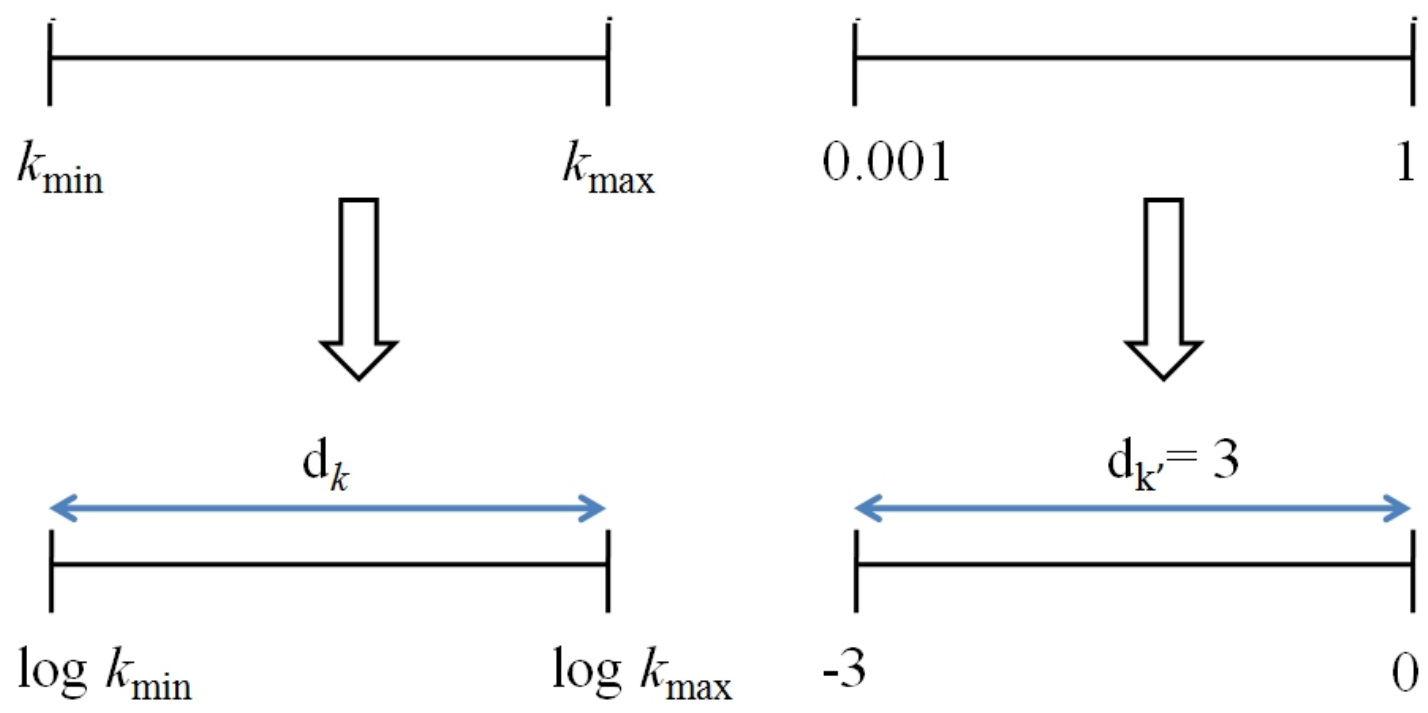

Figure A.3 The range of the rate constants, $k$ and relative rate constants, $k^{\prime}$ on logarithmic scale.

4) Lastly, the positions of the other rate constants are located on the original logarithmic 
scale and they are projected onto the smaller scale. The corresponding relative values, $k^{\prime}$ can then be identified using the formula given. E.g. for the $k$ value of $1.545 \times 10^{9}$, the corresponding $k^{\prime}$ value was calculated to be 0.6 after substituting all the terms in the formula.

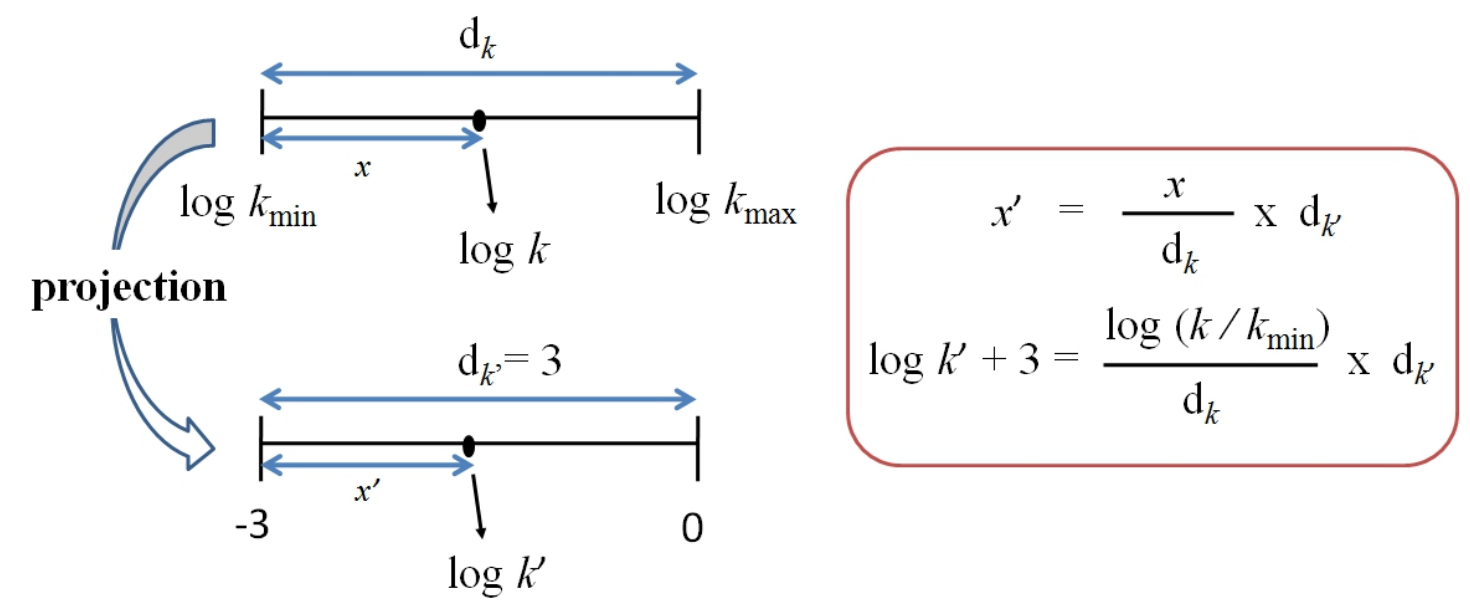

Figure A.4 The $k$ values are projected on the smaller scale and $k^{\prime}$ values are calculated. 


\section{Bibliography}

1. Kadota, J.; Komori, S.; Fukumoto, Y.; Murai, S. J. Org. Chem. 1999, 64, 75237527.

2. Kataoka, Y.; Matsumoto, O.; Tani, K. Organometallics 1996, 15, 5246-5249.

3. Dub, P. A.; Béthegnies, A.; Poli, R. Organometallics 2012, 31, 294-305.

4. Chianese, A. R.; Lee, S. J.; Gagné, M. R. Angew. Chem. Int. Ed. 2007, 46, 40424059.

5. Cao, P.; Cabrera, J.; Padilla, R.; Serra, D.; Rominger, F.; Limbach, M. Organometallics 2012, 31, 921-929.

6. Nakamura, H.; Iwama, H.; Yamamoto, Y. J. Am. Chem. Soc. 1996, 118, 6641-6647.

7. Dub, P. A.; Rodriguez-Zubiri, M.; Daran, J.-C.; Brunet, J.-J.; Poli, R. Organometallics 2009, 28, 4764-4777.

8. Wang, X.; Widenhoefer, R. A. Organometallics 2004, 23, 1649-1651.

9. Brunet, J.-J.; Chu, N.-C.; Rodriguez-Zubiri, M. Eur. J. Inorg. Chem. 2007, 2007, 4711-4722.

10. Brunet, J.-J.; Cadena, M.; Chu, N. C.; Diallo, O.; Jacob, K.; Mothes, E. Organometallics 2004, 23, 1264-1268.

11. Johns, A. M.; Utsunomiya, M.; Incarvito, C. D.; Hartwig, J. F. J. Am. Chem. Soc. 2006, 128, 1828-1839.

12. Yang, M.; Zhang, X.; Lu, X. Org. Lett. 2007, 9, 5131-5133.

13. Trost, B. M.; Simas, A. B. C.; Plietker, B.; Jäkel, C.; Xie, J. Chem-Eur. J. 2005, 11, 7075-7082.

14. Takaya, J.; Iwasawa, N. J. Am. Chem. Soc. 2008, 130, 15254-15255.

15. Jonasson, C.; Horváth, A.; Bäckvall, J.-E. J. Am. Chem. Soc. 2000, 122, 9600-9609.

16. Kadnikov, D. V.; Larock, R. C. J. Org. Chem. 2003, 68, 9423-9432.

17. Solé, D.; Serrano, O. Angew. Chem. Int. Ed. 2007, 46, 7270-7272.

18. Chiusoli, G. P.; Costa, M.; Pallini, L.; Terenghi, G. Transition Met. Chem. 1982, 7, 304-306.

19. Sharma, R. K.; Shoulders, B. A.; Gardner, P. D. J. Org. Chem. 1967, 32, 241-244.

20. Ravindar, K.; Sridhar Reddy, M.; Deslongchamps, P. Org. Lett. 2011, 13, 31783181 . 
21. Nishizawa, M.; Imagawa, H.; Yamamoto, H. Org. Biomol. Chem. 2010, 8, 511-521.

22. Hutchings, G. J. J. Catal. 1985, 96, 292-295.

23. Mizushima, E.; Sato, K.; Hayashi, T.; Tanaka, M. Angew. Chem. Int. Ed. 2002, 41, 4563-4565.

24. Marion, N.; Ramón, R. S.; Nolan, S. P. J. Am. Chem. Soc. 2009, 131, 448-449.

25. Casado, R.; Contel, M.; Laguna, M.; Romero, P.; Sanz, S. J. Am. Chem. Soc. 2003, $125,11925-11935$.

26. Fukuda, Y.; Utimoto, K. J. Org. Chem. 1991, 56, 3729-3731.

27. Fukuda, Y.; Utimoto, K. Bull. Chem. Soc. Jpn. 1991, 64, 2013-2015.

28. Teles, J. H.; Brode, S.; Chabanas, M. Angew. Chem. Int. Ed. 1998, 37, 1415-1418.

29. Roithová, J.; Hrušák, J.; Schröder, D.; Schwarz, H. Inorg. Chim. Acta 2005, 358, 4287-4292.

30. Kovács, G.; Lledós, A.; Ujaque, G. Organometallics 2010, 29, 5919-5926.

31. Kovács, G.; Lledós, A.; Ujaque, G. Angew. Chem. Int. Ed. 2011, 50, 11147-11151.

32. Liu, X.-Y.; Guo, Z.; Dong, S. S.; Li, X.-H.; Che, C.-M. Chem-Eur. J. 2011, 17, 12932-12945.

33. Patil, N. T.; Lakshmi, P. G. V. V.; Singh, V. Eur. J. Org. Chem. 2010, 2010, 47194731 .

34. Li, H.; Widenhoefer, R. A. Org. Lett. 2009, 11, 2671-2674.

35. Chary, B. C.; Kim, S. J. Org. Chem. 2010, 75, 7928-7931.

36. Nishina, N.; Yamamoto, Y. Tetrahedron 2009, 65, 1799-1808.

37. López-Carrillo, V.; Echavarren, A. M. J. Am. Chem. Soc. 2010, 132, 9292-9294.

38. Grirrane, A.; Garcia, H.; Corma, A.; Álvarez, E. ACS Catal. 2011, 1, 1647-1653.

39. Rabaâ, H.; Engels, B.; Hupp, T.; Hashmi, A. S. K. Int. J. Quantum Chem. 2007, $107,359-365$.

40. Montserrat, S.; Alonso, I.; López, F.; Mascareñas, J. L.; Lledós, A.; Ujaque, G. Dalton Trans. 2011, 40, 11095-11105.

41. Sperger, C.; Strand, L. H.; Fiksdahl, A. Tetrahedron 2010, 66, 7749-7754.

42. Muzart, J. Tetrahedron 2008, 64, 5815-5849.

43. Marion, N.; Gealageas, R.; Nolan, S. P. Org. Lett. 2007, 9, 2653-2656. 
44. Kennedy-Smith, J. J.; Staben, S. T.; Toste, F. D. J. Am. Chem. Soc. 2004, 126, 45264527.

45. Hashmi, A. S. K. Chem. Rev. 2007, 107, 3180-3211.

46. Rudolph, M.; Hashmi, A. S. K. Chem. Commun. 2011, 47, 6536-6544.

47. Xu, B.; Wang, W.; Liu, L.-P.; Han, J.; Jin, Z.; Hammond, G. B. J. Organomet. Chem. 2011, 696, 269-276.

48. Rudolph, M.; Hashmi, A. S. K. Chem. Soc. Rev. 2012, 2448-2462.

49. Hashmi, A. S. K. Gold Bull. 2003, 36, 3-9.

50. Leyva-Pérez, A.; Corma, A. Angew. Chem. Int. Ed. 2012, 51, 614-635.

51. Rosenfeld, D. C.; Shekhar, S.; Takemiya, A.; Utsunomiya, M.; Hartwig, J. F. Org. Lett. 2006, 8, 4179-4182.

52. Li, Z.; Zhang, J.; Brouwer, C.; Yang, C.-G.; Reich, N. W.; He, C. Org. Lett. 2006, $8,4175-4178$.

53. Khin, C.; Hashmi, A. S. K.; Rominger, F. Eur. J. Inorg. Chem. 2010, 2010, 10631069.

54. Mézailles, N.; Ricard, L.; Gagosz, F. Org. Lett. 2005, 7, 4133-4136.

55. Ricard, L.; Gagosz, F. Organometallics 2007, 26, 4704-4707.

56. Leyva, A.; Corma, A. J. Org. Chem. 2009, 74, 2067-2074.

57. Gómez-Suárez, A.; Oonishi, Y.; Meiries, S.; Nolan, S. P. Organometallics 2013, 32 , 1106-1111.

58. Hashmi, A. S. K. Angew. Chem. Int. Ed. 2000, 39, 3590-3593.

59. Schmidbaur, H.; Schier, A. Arab. J. Sci. Eng. 2012, 37, 1187-1225.

60. Lemière, G.; Gandon, V.; Agenet, N.; Goddard, J.-P.; de Kozak, A.; Aubert, C.; Fensterbank, L.; Malacria, M. Angew. Chem. Int. Ed. 2006, 45, 7596-7599.

61. Zriba, R.; Gandon, V.; Aubert, C.; Fensterbank, L.; Malacria, M. Chem-Eur. J. 2008, $14,1482-1491$.

62. Cheong, P. H.-Y.; Morganelli, P.; Luzung, M. R.; Houk, K. N.; Toste, F. D. J. Am. Chem. Soc. 2008, 130, 4517-4526.

63. Manzo, A. M.; Perboni, A. D.; Broggini, G.; Rigamonti, M. Tetrahedron Lett. 2009, 50, 4696-4699.

64. Yang, C.-Y.; Lin, G.-Y.; Liao, H.-Y.; Datta, S.; Liu, R.-S. J. Org. Chem. 2008, 73, 4907-4914. 
65. Hashmi, A. S. K. Angew. Chem. Int. Ed. 2005, 44, 6990-6993.

66. Gorin, D. J.; Toste, F. D. Nature 2007, 446, 395-403.

67. Zhang, X.; Corma, Chem. Commun. 2007, 3080-3082.

68. Zhang, X.; Corma, A. Dalton Trans. 2007, 397-403.

69. Zhang, X.; Corma, A. Angew. Chem. Int. Ed. 2008, 47, 4358-4361.

70. Roembke, P.; Schmidbaur, H.; Cronje, S.; Raubenheimer, H. J. Mol. Catal. A: Chem. 2004, 212, 35-42.

71. Graf, T. A.; Anderson, T. K.; Bowden, N. B. Adv. Synth. Catal. 2011, 353, $1033-$ 1038.

72. Hashmi, A. S. K.; Hutchings, G. J. Angew. Chem. Int. Ed. 2006, 45, 7896-7936.

73. Marion, N.; Nolan, S. P. Chem. Soc. Rev. 2008, 37, 1776-1782.

74. Kuhn, N.; Al-Sheikh, A. Coord. Chem. Rev. 2005, 249, 829-857.

75. Díez-González, S.; Marion, N.; Nolan, S. P. Chem. Rev. 2009, 109, 3612-3676.

76. Blake, G. A.; Moerdyk, J. P.; Bielawski, C. W. Organometallics 2012, 31, 33733378 .

77. Rosen, E. L.; Varnado, C. D.; Tennyson, A. G.; Khramov, D. M.; Kamplain, J. W.; Sung, D. H.; Cresswell, P. T.; Lynch, V. M.; Bielawski, C. W. Organometallics 2009, 28, 6695-6706.

78. Alvarado, E.; Badaj, A. C.; Larocque, T. G.; Lavoie, G. G. Chem-Eur. J. 2012, 18, 12112-12121.

79. Almássy, A.; Nagy, C. E.; Bényei, A. C.; Joó, F. Organometallics 2010, 29, 24842490.

80. Sanz, S.; Jones, L. A.; Mohr, F.; Laguna, M. Organometallics 2007, 26, 952-957.

81. Marion, N. et al. Chem-Eur. J. 2009, 15, 3243-3260.

82. Mamane, V.; Gress, T.; Krause, H.; Fürstner, A. J. Am. Chem. Soc. 2004, 126, 86548655 .

83. Kleinbeck, F.; Toste, F. D. J. Am. Chem. Soc. 2009, 131, 9178-9179.

84. Seidel, G.; Mynott, R.; Fürstner, A. Angew. Chem. 2009, 121, 2548-2551.

85. Fürstner, A.; Morency, L. Angew. Chem. 2008, 120, 5108-5111.

86. Hashmi, A. S. K. Angew. Chem. Int. Ed. 2008, 47, 6754-6756.

87. Fürstner, A.; Davies, P. W. Angew. Chem. Int. Ed. 2007, 46, 3410-3449. 
88. Benitez, D.; Shapiro, N. D.; Tkatchouk, E.; Wang, Y.; Goddard III, W. A.; Toste, F. D. Nat. Chem. 2009, 1, 482-486.

89. Velegraki, G.; Stratakis, M. J. Org. Chem. 2013, 78, 8880-8884.

90. Gorin, D. J.; Sherry, B. D.; Toste, F. D. Chem. Rev. 2008, 108, 3351-3378.

91. Corma, A.; Ruiz, V. R.; Leyva-Pérez, A.; Sabater, M. J. Adv. Synth. Catal. 2010, $352,1701-1710$.

92. Hesp, K. D.; Stradiotto, M. J. Am. Chem. Soc. 2010, 132, 18026-18029.

93. Wang, T.; Shi, S.; Vilhelmsen, M. H.; Zhang, T.; Rudolph, M.; Rominger, F.; Hashmi, A. S. K. Chem-Eur. J. 2013, 19, 12512-12516.

94. Zi, W.; Toste, F. D. J. Am. Chem. Soc. 2013, 135, 12600-12603.

95. Hamilton, G. L.; Kang, E. J.; Mba, M.; Toste, F. D. Science 2007, 317, 496-499.

96. Norrby, L. J. J. Chem. Educ. 1991, 68, 110.

97. Pouy, M. J.; Delp, S. A.; Uddin, J.; Ramdeen, V. M.; Cochrane, N. A.; Fortman, G. C.; Gunnoe, T. B.; Cundari, T. R.; Sabat, M.; Myers, W. H. ACS Catal. 2012, 2, 2182-2193.

98. Thuong, M. B. T.; Mann, A.; Wagner, A. Chem. Commun. 2012, 48, 434-436.

99. Tokunaga, M.; Wakatsuki, Y. Angew. Chem. Int. Ed. 1998, 37, 2867-2869.

100. Grotjahn, D. B.; Lev, D. A. J. Am. Chem. Soc. 2004, 126, 12232-12233.

101. Labonne, A.; Kribber, T.; Hintermann, L. Org. Lett. 2006, 8, 5853-5856.

102. Pernpointner, M.; Hashmi, A. S. K. J. Chem. Theory Comput. 2009, 5, 2717-2725.

103. Hashmi, A. S. K.; Pernpointner, M.; Hansmann, M. M. Faraday Discuss. 2011, 152, 179-184.

104. Pyykkö, P.; Desclaux, J. P. Acc. Chem. Res. 1979, 12, 276-281.

105. Desclaux, J. P.; Pyykkö, P. Chem. Phys. Lett. 1976, 39, 300-303.

106. Pyykkö, P. Chem. Rev. 1988, 88, 563-594.

107. Hrušák, J.; Hertwig, R. H.; Schröder, D.; Schwerdtfeger, P.; Koch, W.; Schwarz, H. Organometallics 1995, 14, 1284-1291.

108. Pyykkö, P. Angew. Chem. Int. Ed. 2002, 41, 3573-3578.

109. Schwerdtfeger, P. Heteroat. Chem. 2002, 13, 578-584.

110. Nakanishi, W.; Yamanaka, M.; Nakamura, E. J. Am. Chem. Soc. 2005, 127, 14461453. 
111. Hertwig, R. H.; Koch, W.; Schröder, D.; Schwarz, H.; Hrušák, J.; Schwerdtfeger, P. J. Phys. Chem. 1996, 100, 12253-12260.

112. Nechaev, M. S.; Rayón, V. M.; Frenking, G. J. Phys. Chem. A 2004, 108, 3134 3142 .

113. de Frémont, P.; Scott, N. M.; Stevens, E. D.; Nolan, S. P. Organometallics 2005, 24, 2411-2418.

114. Pyykkö, P. Annu. Rev. Phys. Chem. 2012, 63, 45-64.

115. Krauter, C. M.; Hashmi, A. S. K.; Pernpointner, M. ChemCatChem 2010, 2, 12261230.

116. Mazzone, G.; Russo, N.; Sicilia, E. Organometallics 2012, 31, 3074-3080.

117. Lein, M.; Rudolph, M.; Hashmi, S. K.; Schwerdtfeger, P. Organometallics 2010, 29 , 2206-2210.

118. Hashmi, A. S. K. Catal. Today 2007, 122, 211-214.

119. Hashmi, A. S. K.; Schwarz, L.; Choi, J.-H.; Frost, T. M. Angew. Chem. Int. Ed. 2000, 39, 2285-2288.

120. Hashmi, A. S. K.; Weyrauch, J. P.; Frey, W.; Bats, J. W. Org. Lett. 2004, 6, 43914394.

121. Mazzone, G.; Russo, N.; Sicilia, E. J. Chem. Theory Comput. 2010, 6, 2782-2789.

122. Grimme, S.; Antony, J.; Ehrlich, S.; Krieg, H. J. Chem. Phys. 2010, 132, 154104.

123. Grimme, S.; Ehrlich, S.; Goerigk, L. J. Comput. Chem. 2011, 32, 1456-1465.

124. Kang, R.; Chen, H.; Shaik, S.; Yao, J. J. Chem. Theory Comput. 2011, 7, 4002-4011.

125. Perdew, J. P.; Burke, K.; Ernzerhof, M. Phys. Rev. Lett. 1996, 77, 3865-3868.

126. Perdew, J. P.; Burke, K.; Ernzerhof, M. Phys. Rev. Lett. 1997, 78, 1396.

127. Adamo, C.; Barone, V. J. Chem. Phys. 1999, 110, 6158-6159.

128. Becke, A. D. Phys. Rev. A 1988, 38, 3098-3100.

129. Perdew, J. P. Phys. Rev. B 1986, 33, 8822-8824.

130. Douglas, M.; Kroll, N. M. Ann. Phys. 1974, 82, 89-155.

131. Hess, B. A. Phys. Rev. A 1985, 32, 756-763.

132. Hess, B. A. Phys. Rev. A 1986, 33, 3742-3748.

133. Jansen, G.; Hess, B. A. Phys. Rev. A 1989, 39, 6016-6017.

134. Feller, D. J. Comp. Chem. 1996, 17, 1571-1586. 
135. Schuchardt, K. L.; Didier, B. T.; Elsethagen, T.; Sun, L.; Gurumoorthi, V.; Chase, J.; Li, J.; Windus, T. J. Chem. Inf. Model. 2007, 47, 1045-1052.

136. Dunning, T. H. J. Chem. Phys. 1989, 90, 1007.

137. Woon, D. E.; Dunning, T. H. J. Chem. Phys. 1993, 98, 1358.

138. Figgen, D.; Rauhut, G.; Dolg, M.; Stoll, H. Chem. Phys. 2005, 311, 227.

139. Peterson, K. A.; Puzzarini, C. Theor. Chem. Acc. 2005, 114, 283.

140. Frisch, M. J. et al. Gaussian 09 Revision D.01, Gaussian Inc. Wallingford CT 2009.

141. Schlegel, H. B. J. Comp 1982, 3, 214-218.

142. Li, X.; Frisch, M. J. J. Chem. Theory Comput. 2006, 2, 835-839.

143. Hratchian, H. P.; Schlegel, H. B. J. Chem. Phys. 2004, 120, 9918-9924.

144. Hratchian, H. P.; Schlegel, H. B. J. Chem. T 2005, 1, 61-69.

145. Cancès, E.; Mennucci, B.; Tomasi, J. J. Chem. Phys. 1997, 107, 3032-3041.

146. Cossi, M.; Scalmani, G.; Rega, N.; Barone, V. J. Chem. Phys. 2002, 117, 43.

147. Jensen, F. Introduction to Computational Chemistry; John Wiley \& Sons, Inc., 2013; pp 421-444.

148. Wolfram Research, I., Inc.Wolfram Research Mathematica 10.0, 2014, Wolfram Research, Inc. 